\title{
Benzylic C-H Azidation Using the Zhdankin Reagent and a Copper Photoredox Catalyst
}

\author{
Pauline T. G. Rabet ${ }^{\dagger}$, Gabriele Fumagalli, ${ }^{\dagger}$ Scott Boyd ${ }^{\dagger}$, Michael F. Greaney ${ }^{\dagger} *$ \\ ${ }^{\dagger}$ School of Chemistry, University of Manchester, Oxford Road, Manchester, UK, M13 9PL, UK \\ ${ }^{\star}$ Department of Oncology, AstraZeneca, Alderley Park, Macclesfield, SK10 4TG, UK
}

\section{Supporting information}

GENERAL INFORMATIONNATISISATION




\section{General information}

${ }^{1} \mathrm{H}-\mathrm{NMR},{ }^{13} \mathrm{C}\left\{{ }^{1} \mathrm{H}\right\}-\mathrm{NMR}$ and ${ }^{19} \mathrm{~F}-\mathrm{NMR}$ were recorded at 500/400 MHz, $125 / 100 \mathrm{MHz}, 470 / 376 \mathrm{MHz}$ on a Bruker 500 or 400 spectrometers. All spectra are referenced to $\mathrm{CDCl}_{3}$ residual $\mathrm{CHCl}_{3}$ peak $\left({ }^{1} \mathrm{H}-\mathrm{NMR} \delta=\right.$ $7.26 \mathrm{ppm} ;{ }^{13} \mathrm{C}\left\{{ }^{1} \mathrm{H}\right\}-\mathrm{NMR} \delta=77.16 \mathrm{ppm}$ ). All chemical shifts are quoted in parts per million (ppm), measured from the centre of the signal except in the case of multiplets of more than one proton, which are quoted as a range. Coupling constants are quoted to the nearest $0.1 \mathrm{~Hz}$. Splitting patterns are abbreviated as follows: singlet (s), doublet (d), triplet (t), quartet (q), quintet (quin.), heptet (hept.), multiplet (m), broad singlet (brs) and combinations thereof.

Infrared spectra were recorded on a spectrometer as neat using a Perkin-Elmer FT-IR Spectrum RX1 or BX spectrometers.

Low resolution mass spectrometry was performed on an Agilent 6100 mass spectrometer (ESI or APCI ionisation) and Hewlett Packard 5971 MSD (GC/MS with EI). High resolution mass spectrometry was performed on a Waters QTOF with ESI/APCI ionisation and a Thermo Finnigan MAT95XP (EI). Thin layer chromatography (TLC) was performed using pre-coated Merck 60F254 silica plates. Visualization was performed using either UV light or treatment with acidic potassium permanganate. Flash chromatography was performed using Merck Kieselgel (mesh size 220-240) silica.

All reagents and solvents were used as obtained from commercial source, unless otherwise stated. Solvents were degassed by bubbling nitrogen gas through them for twenty minutes prior to use. 1-azido-1 $\lambda^{3}$ benzo[ $d][1,2]$ iodaoxol-3( $1 H)$-one, ${ }^{1} \quad \mathrm{Cu}(\mathrm{dap})_{2} \mathrm{Cl}^{2}{ }^{2}$ and tert-butyl 2 -methyl-1H-indole-1-carboxylate ${ }^{3}$ were synthesised according to literature procedures.

Safety: Azides are potentially explosive compounds and require appropriate safety protocols to be observed at all times. ${ }^{4}$

N.B. Whilst 1-azido- $1 \lambda^{3}$-benzo[d][1,2]iodaoxol-3(1H)-one is more stable than other iodine azide reagents, it is reported to decompose with explosion at $138-139^{\circ} \mathrm{C}^{5}$ 


\section{Optimisation}

\section{Catalyst screening}

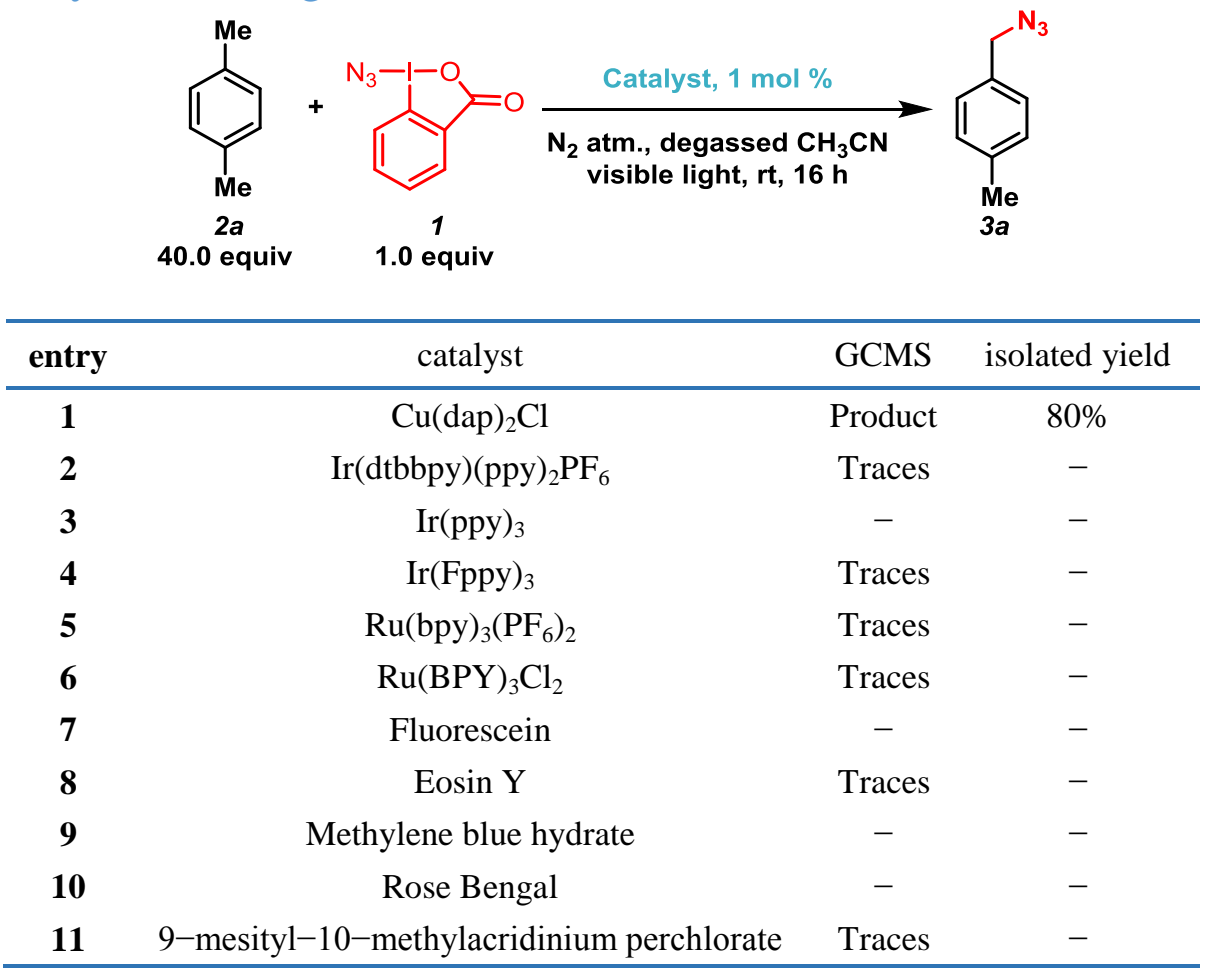

\section{Table 1}

A vial containing the photoredox catalyst (0.01 equiv, $0.005 \mathrm{mmol}, 4.40 \mathrm{mg}), 1-$ azido-1 $\lambda^{3}-$ benzo[d][1,2]iodaoxol-3(1H)-one (1.00 equiv, $0.50 \mathrm{mmol}, 145 \mathrm{mg})$ and equipped with a stirrer bar, sealed with a septum, was purged under a flux of nitrogen. Then degassed mixture of acetonitrile $(2.5 \mathrm{~mL})$ and para-xylene (40.0 equiv, $20.0 \mathrm{mmol}, 2.50 \mathrm{~mL}$ ) were added and the tube was sealed and put one centimeter away from a 30 W fluorescent bulb and stirred for 16 hours. The crude reaction mixture was loaded directly on the chromatography column and purified with mixtures of hexane:DCM.

\section{Control experiments}<smiles>Cc1ccc(C)cc1</smiles>

$2 a$

40.0 equiv<smiles>N#I1OC(=O)c2ccccc21</smiles>

1
Copper source, $\mathbf{0 . 5} \mathbf{~ m o l} \%$

$\mathrm{N}_{2}$ atm., degassed $\mathrm{CH}_{3} \mathrm{CN}$ visible light, $\mathrm{rt}, 16 \mathrm{~h}$

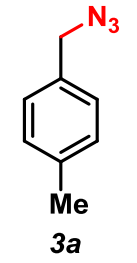

1.0 equiv

\begin{tabular}{ccccc}
\hline entry & $\mathrm{Cu}(\text { dap })_{2} \mathrm{Cl}$ & $\mathrm{CuBr}$ & light & isolated Yield \\
\hline $\mathbf{1}$ & $\mathrm{Y}$ & $\mathrm{N}$ & $\mathrm{Y}$ & $80 \%$ \\
$\mathbf{2}$ & $\mathrm{Y}$ & $\mathrm{N}$ & $\mathrm{N}$ & $29 \%^{\mathrm{a}}$
\end{tabular}




\begin{tabular}{ccccc}
$\mathbf{3}$ & $\mathrm{N}$ & $\mathrm{Y}$ & $\mathrm{Y}$ & $55 \%^{\mathrm{a}}$ \\
$\mathbf{4}$ & $\mathrm{N}$ & $\mathrm{Y}$ & $\mathrm{N}$ & Traces \\
$\mathbf{5}$ & $\mathrm{N}$ & $\mathrm{N}$ & $\mathrm{Y}$ & $17 \%^{\mathrm{b}}$ \\
$\mathbf{6}$ & $\mathrm{N}$ & $\mathrm{N}$ & $\mathrm{N}$ & $0 \%$ \\
$\mathbf{7}$ & $\mathrm{N}$ & $\mathrm{Y}$ & $0 \%^{\mathrm{c}}$ \\
$\mathbf{8}$ & $\mathrm{N}$ & $\mathrm{Y}$ & $0 \%^{\mathrm{d}}$ \\
\hline${ }^{\mathrm{a}}$ Average yield of two reactions & ${ }^{\mathrm{b}}$ Average yield of three reactions \\
\multicolumn{4}{c}{1.0 equiv TEMPO was added to the reaction mixture }
\end{tabular}

Table 2

\begin{tabular}{ccc}
\hline Entry & $\mathrm{CuBr}(\mathrm{mol} \%)$ & Isolated Yield \\
\hline $\mathbf{1}$ & 0.5 & $55 \%$ \\
$\mathbf{2}$ & 1 & $36 \%$ \\
$\mathbf{3}$ & 2 & $38 \%$ \\
$\mathbf{4}$ & 5 & $28 \%$ \\
\hline
\end{tabular}

Table 3

\section{Reactions run under visible light irradiation}

A vial containing the copper source, 1 -azido- $1 \lambda^{3}$-benzo[ $\left.d\right][1,2]$ iodaoxol-3(1H)-one (1.00 equiv, $0.50 \mathrm{mmol}$, $145 \mathrm{mg}$ ) and equipped with a stirrer bar, sealed with a septum, was purged under a flux of nitrogen. Then degassed mixture of acetonitrile $(2.5 \mathrm{~mL})$ and para-xylene $(40.0$ equiv, $20.0 \mathrm{mmol}, 2.50 \mathrm{~mL})$ were added and the tube was sealed and put one centimeter away from a $30 \mathrm{~W}$ fluorescent bulb and stirred for 16 hours. The crude reaction mixture was loaded directly on the chromatography column and purified with mixtures of hexane:DCM.

\section{Reactions run in the dark}

A vial containing the copper source, 1 -azido- $1 \lambda^{3}$-benzo[ $\left.d\right][1,2]$ iodaoxol-3(1H)-one (1.00 equiv, $0.50 \mathrm{mmol}$, $145 \mathrm{mg}$ ) and equipped with a stirrer bar, sealed with a septum and wrapped in tin foil, was purged under a flux of nitrogen. Then degassed mixture of acetonitrile $(2.5 \mathrm{~mL})$ and para-xylene (40.0 equiv, $20.0 \mathrm{mmol}, 2.50 \mathrm{~mL}$ ) were added and the tube was sealed and stirred for 16 hours. The crude reaction mixture was loaded directly on the chromatography column and purified with mixtures of hexane:DCM. 


\section{Characterisation for azidated substrates $3 a$ to 4}

1-(azidomethyl)-4-methylbenzene (3a)

1

A vial containing $\mathrm{Cu}(\mathrm{dap})_{2} \mathrm{Cl} \quad(0.005$ equiv, $0.003 \mathrm{mmol}, 2.20 \mathrm{mg}), \quad 1-$ azido-1 $\lambda^{3}-$ benzo[ $d][1,2]$ iodaoxol-3(1H)-one (1.00 equiv, $0.50 \mathrm{mmol}, 145 \mathrm{mg}$ ) and equipped with a stirrer bar, sealed with a septum, was purged under a flux of nitrogen. Then degassed mixture of acetonitrile $(2.5 \mathrm{~mL})$ and para-xylene (40.0 equiv, $20.0 \mathrm{mmol}, 2.50 \mathrm{~mL}$ ) was added and the tube was sealed and put one centimeter away from a $30 \mathrm{~W}$ fluorescent bulb and stirred for 16 hours. The crude reaction mixture was loaded directly on the chromatography column and purified with mixtures of hexane:DCM affording $3 \boldsymbol{a}$ as a colourless oil (59.0 mg, 80\%). Data are in accordance with previous literature reports. ${ }^{16}{ }^{1} \mathrm{H}$ NMR (400 $\mathrm{MHz}$, Chloroform-d) $\delta 7.23-7.18(\mathrm{~m}, 4 \mathrm{H}), 4.29$ (s, 2H), 2.37 (s, 3H). ${ }^{13} \mathrm{C}$ NMR (101 MHz, Chloroform-d) $\delta$ 138.3, 132.4, 129.6, 128.4, 54.8, 21.3. IR (neat film): 2923, 2092, 1515, 1447, 1252, 802, 753, 734. MS (EI): $147(\mathrm{M}), 118.1\left(\mathrm{M}-\mathrm{N}_{2}\right), 105\left(\mathrm{M}-\mathrm{N}_{3}\right), 91\left(\mathrm{M}-\mathrm{CH}_{2} \mathrm{~N}_{3}\right)$. HRMS (EI): Calculated for $\mathrm{C}_{8} \mathrm{H}_{9} \mathrm{~N}_{3}$ : 147.0791; Found: 147.0794.

(azidomethyl)benzene $(3 \boldsymbol{b})$

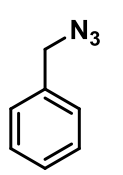

A vial containing $\mathrm{Cu}(\text { dap })_{2} \mathrm{Cl}(0.005$ equiv, $0.003 \mathrm{mmol}, 2.20 \mathrm{mg}), \quad 1$-azido-1 $\lambda^{3}-$ benzo[d][1,2]iodaoxol-3(1H)-one (1.00 equiv, $0.50 \mathrm{mmol}, 145 \mathrm{mg})$ and equipped with a stirrer bar, sealed with a septum, was purged under a flux of nitrogen. Then degassed mixture of acetonitrile $(2.5 \mathrm{~mL})$ and toluene (40.0 equiv, $20.0 \mathrm{mmol}, 2.10 \mathrm{~mL})$ was added and the tube was sealed and put one centimeter away from a $30 \mathrm{~W}$ fluorescent bulb and stirred for 16 hours. The crude reaction mixture was loaded directly on the chromatography column and purified with mixtures of hexane:DCM affording $\mathbf{3} \boldsymbol{b}$ as a colourless oil (41.0 mg, 61\%). Data are in accordance with previous literature reports. ${ }^{6}{ }^{1} \mathrm{H}$ NMR (400 MHz, Chloroform-d) $\delta 7.43-7.30(\mathrm{~m}, 5 \mathrm{H}), 4.35$ (s, 2H). ${ }^{13} \mathrm{C}$ NMR (101 MHz, Chloroform-d) $\delta 135.5,129.0,128.5$, 128.4, 55.0. IR (neat film): 3032, 2091, 1600, 1496, 1455, 1253, 734, 696. MS (EI): 133 (M), $91\left(\mathrm{M}-\mathrm{N}_{3}\right)$. HRMS (EI): Calculated for $\mathrm{C}_{7} \mathrm{H}_{7} \mathrm{~N}_{3}$ (M): 133.0634; Found: 133.0637.

1-(azidomethyl)-2-methylbenzene (3c)

$\mathrm{N}_{3} \mathrm{~A}$ vial containing $\mathrm{Cu}(\mathrm{dap})_{2} \mathrm{Cl}(0.005$ equiv, $0.003 \mathrm{mmol}, 2.20 \mathrm{mg}), \quad 1-$ azido-1 $\lambda^{3}-$ benzo[d][1,2]iodaoxol-3(1H)-one (1.00 equiv, $0.50 \mathrm{mmol}, 145 \mathrm{mg})$ and equipped with a stirrer bar, sealed with a septum, was purged under a flux of nitrogen. Then degassed mixture of acetonitrile $(2.5 \mathrm{~mL})$ and ortho-xylene (40.0 equiv, $20.0 \mathrm{mmol}, 2.50 \mathrm{~mL}$ ) was added and the tube was sealed and put one centimeter away from a $30 \mathrm{~W}$ fluorescent bulb and stirred for 16 hours. The crude reaction mixture was loaded directly on the chromatography column and purified with mixtures of hexane:DCM affording $3 c$ as a colourless oil $(57.0 \mathrm{mg}, 77 \%)$. Data are in accordance with previous literature reports. ${ }^{9}{ }^{1} \mathrm{H}$ NMR (400 MHz, Chloroform-d) $\delta 7.29-7.21(\mathrm{~m}, 4 \mathrm{H}), 4.35$ (s, 2H), 2.37 (s, 3H). ${ }^{13} \mathrm{C} \mathrm{NMR}(101 \mathrm{MHz}$, Chloroform-d) $\delta 136.9,133.5,130.8,129.5,128.8,126.3,53.2,19.1$. IR (neat film): 2930, 2094, 1461, 1247 , 741. MS (EI): $147(\mathrm{M}), 119\left(\mathrm{M}-\mathrm{N}_{2}\right), 105\left(\mathrm{M}-\mathrm{N}_{3}\right), 91\left(\mathrm{M}-\mathrm{CH}_{2} \mathrm{~N}_{3}\right)$. HRMS (APCI +): Calculated for $\mathrm{C}_{8} \mathrm{H}_{10} \mathrm{~N}\left(\mathrm{M}-\mathrm{N}_{2}+\mathrm{H}^{+}\right)$: 120.0808; Found: 149.0813. 
1-(azidomethyl)-3-methylbenzene (3d)

$\mathrm{N}_{3} \mathrm{~A}$ vial containing $\mathrm{Cu}(\mathrm{dap})_{2} \mathrm{Cl}(0.005$ equiv, $0.003 \mathrm{mmol}, 2.20 \mathrm{mg}), \quad 1$-azido- $-1 \lambda^{3}-$
benzo[d][1,2]iodaoxol-3(1H)-one (1.00 equiv, $0.50 \mathrm{mmol}, 145 \mathrm{mg})$ and equipped with a stirrer
bar, sealed with a septum, was purged under a flux of nitrogen. Then degassed mixture of acetonitrile $(2.5 \mathrm{~mL})$ and $m e t a-x y l e n e(40.0$ equiv, $20.0 \mathrm{mmol}, 2.50 \mathrm{~mL})$ was added and the tube was sealed and put one centimeter away from a $30 \mathrm{~W}$ fluorescent bulb and stirred for 16 hours. The crude reaction mixture was loaded directly on the chromatography column and purified with mixtures of hexane:DCM affording $3 \boldsymbol{d}$ as a colourless oil $(53.0 \mathrm{mg}, 60 \%)$. Data are in accordance with previous literature reports. ${ }^{9} \mathrm{H}$ NMR (400 MHz, Chloroform-d) $\delta 7.29(\mathrm{~m}, 1 \mathrm{H}), 7.18-7.11(\mathrm{~m}, 3 \mathrm{H}), 4.31(\mathrm{~s}, 2 \mathrm{H}), 2.38(\mathrm{~s}, 3 \mathrm{H}) .{ }^{13} \mathrm{C}$ NMR (101 MHz, Chloroform- $d$ ) $\delta 138.7,135.4,129.2,129.1,128.8,125.4,55.0,21.5$. IR (neat film): 2923, 2092, 1263, 1234, 777, 740, 699. MS (EI): $147(\mathrm{M}), 119\left(\mathrm{M}-\mathrm{N}_{2}\right), 91\left(\mathrm{M}-\mathrm{CH}_{2} \mathrm{~N}_{3}\right)$. HRMS (APCI +): Calculated for $\mathrm{C}_{8} \mathrm{H}_{10} \mathrm{~N}\left(\mathrm{M}-\mathrm{N}_{2}+\mathrm{H}^{+}\right)$: 120.0808; Found: 120.0813 .

1-(azidomethyl)-2-bromobenzene (3e)

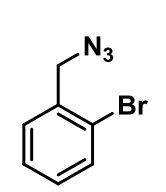

A vial containing $\mathrm{Cu}(\mathrm{dap})_{2} \mathrm{Cl} \quad(0.005$ equiv, 0.003 mmol, $2.20 \mathrm{mg}), \quad 1$-azido- $1 \lambda^{3}-$ benzo[ $d][1,2]$ iodaoxol-3(1H)-one (1.00 equiv, $0.50 \mathrm{mmol}, 145 \mathrm{mg})$ and equipped with a stirrer bar, sealed with a septum, was purged under a flux of nitrogen. Then degassed mixture of acetonitrile $(2.5 \mathrm{~mL})$ and 1-bromo-2-methylbenzene ( 40.0 equiv, $20.0 \mathrm{mmol}, 2.40 \mathrm{~mL}$ ) was added and the tube was sealed and put one centimeter away from a $30 \mathrm{~W}$ fluorescent bulb and stirred for 16 hours. The crude reaction mixture was loaded directly on the chromatography column and purified with mixtures of hexane:DCM affording $3 \boldsymbol{e}$ as a colourless oil $(86.0 \mathrm{mg}, 81 \%) .{ }^{16} \mathrm{H}$ NMR $(400 \mathrm{MHz}$, Chloroform- $d$ ) $\delta 7.61$ (dd, $J=8.0,1.2$ $\mathrm{Hz}, 1 \mathrm{H}$ ), 7.41-7.33 (m, 2H), 7.21 (app td, $J=7.7,1.9 \mathrm{~Hz}, 1 \mathrm{H}$ ), 4.50 (s, 2H). ${ }^{13} \mathrm{C}$ NMR (126 MHz, Chloroformd) $\delta 134.9,133.1,130.1,130.0,127.9,123.8,54.7$. IR (neat film): 2093, 1441, 1342, 1283, 1028, 748. MS (EI): $213\left({ }^{81} \mathrm{BrM}\right), 211\left({ }^{79} \mathrm{BrM}\right), 185\left({ }^{81} \mathrm{BrM}-\mathrm{N}_{2}\right), 183\left({ }^{79} \mathrm{BrM}-\mathrm{N}_{2}\right), 171\left({ }^{81} \mathrm{BrM}-\mathrm{N}_{3}\right), 169\left({ }^{79} \mathrm{BrM}-\mathrm{N}_{3}\right), 157$ $\left({ }^{81} \mathrm{BrM}-\mathrm{CH}_{2} \mathrm{~N}_{3}\right), 155\left({ }^{79} \mathrm{BrM}-\mathrm{CH}_{2} \mathrm{~N}_{3}\right), 104\left({ }^{81} \mathrm{BrM}-\mathrm{Br}-\mathrm{N}_{2}\right), 76\left(\mathrm{M}-\mathrm{Br}-\mathrm{CH}_{2} \mathrm{~N}_{3}\right)$. HRMS (APCI + ): Calculated for $\mathrm{C}_{7} \mathrm{H}_{7} \mathrm{NBr}\left(\mathrm{M}-\mathrm{N}_{2}+\mathrm{H}^{+}\right)$: 183.9756; Found: 183.9757 .

1-(azidomethyl)-2-iodobenzene ( $3 f$ )

$\mathrm{N}_{3} \mathrm{~A}$ vial containing $\mathrm{Cu}(\mathrm{dap})_{2} \mathrm{Cl} \quad(0.005$ equiv, $0.003 \quad \mathrm{mmol}, \quad 2.20 \mathrm{mg}), \quad 1$-azido- $-1 \lambda^{3}-$
benzo $[d][1,2]$ iodaoxol-3(1H)-one $(1.00$ equiv, $0.50 \mathrm{mmol}, 145 \mathrm{mg})$ and equipped with a stirrer bar,
sealed with a septum, was purged under a flux of nitrogen. Then degassed mixture of acetonitrile $(2.5$ $\mathrm{mL}$ ) and 1-iodo-2-methylbenzene ( 40.0 equiv, $20.0 \mathrm{mmol}, 2.55 \mathrm{~mL}$ ) was added and the tube was sealed and put one centimeter away from a $30 \mathrm{~W}$ fluorescent bulb and stirred for 16 hours. The crude reaction mixture was loaded directly on the chromatography column and purified with mixtures of hexane:DCM affording $3 f$ as a colourless oil $(29.0 \mathrm{mg}, 22 \%)$. Data are in accordance with previous literature reports. ${ }^{17} \mathrm{H}$ NMR $(400 \mathrm{MHz}$, Chloroform- $d$ ) $\delta 7.88(\mathrm{~d}, J=8.0,1 \mathrm{H}), 7.38(\mathrm{~d}, J=4.4 \mathrm{~Hz}, 2 \mathrm{H}), 7.04($ app td, $J=8.0,4.4 \mathrm{~Hz}, 1 \mathrm{H}), 4.46(\mathrm{~s}, 2 \mathrm{H})$. ${ }^{13} \mathrm{C}$ NMR (101 MHz, Chloroform-d) $\delta 139.9,138.2,130.1,129.6,128.8$, 99.1, 59.2. IR (neat film): 2961, 2092, 1258, 1014, 793, 748. MS (EI): 259 (M), $231\left(\mathrm{M}-\mathrm{N}_{2}\right), 203\left(\mathrm{M}-\mathrm{CH}_{2} \mathrm{~N}_{3}\right), 104\left(\mathrm{M}-\mathrm{I}-\mathrm{N}_{2}\right), 76(\mathrm{M}-\mathrm{I}-$ $\mathrm{CH}_{2} \mathrm{~N}_{3}$ ). HRMS (APCI + ): Calculated for $\mathrm{C}_{7} \mathrm{H}_{7} \mathrm{NI}\left(\mathrm{M}-\mathrm{N}_{2}+\mathrm{H}^{+}\right)$: 231.9618 ; Found: 231.9612. 
$\mathrm{N}_{3} \mathrm{~A}$ vial containing $\mathrm{Cu}(\mathrm{dap})_{2} \mathrm{Cl}(0.005$ equiv, $0.003 \mathrm{mmol}, 2.20 \mathrm{mg}), \quad 1$-azido- $-1 \lambda^{3}-$
benzo $[d][1,2]$ iodaoxol- $-3(1 H)$-one $(1.00$ equiv, $0.50 \mathrm{mmol}, 145 \mathrm{mg})$ and equipped with a stirrer
bar, sealed with a septum, was purged under a flux of nitrogen. Then degassed mixture of acetonitrile $(2.5 \mathrm{~mL})$ and 2-methyl-1,1'-biphenyl (40.0 equiv, $20.0 \mathrm{mmol}, 3.30 \mathrm{~mL}$ ) was added and the tube was sealed and put one centimeter away from a $30 \mathrm{~W}$ fluorescent bulb and stirred for 16 hours. The crude reaction mixture was loaded directly on the chromatography column and purified with mixtures of hexane:DCM affording $3 g$ as a colourless oil $(59.0 \mathrm{mg}, 60 \%)$. Data are in accordance with previous literature reports. ${ }^{18}{ }^{1} \mathrm{H}$ NMR (400 MHz, Chloroform-d) $\delta$ 7.48-7.33 (m, 9H), 4.30 (s, 2H). ${ }^{13} \mathrm{C}$ NMR (101 MHz, Chloroform-d) $\delta$ 142.3, 140.4, 132.9, 130.6, 129.7, 129.3, 128.5, 128.5, 127.9, 127.6, 52.7. IR (neat film): 3061, 2089, 1479, 1252, 1009, 745, 701, 617. MS (APCI +): $182\left(\mathrm{M}-\mathrm{N}_{2}+\mathrm{H}^{+}\right)$. HRMS (APCI + ): Calculated for $\mathrm{C}_{13} \mathrm{H}_{12} \mathrm{~N}(\mathrm{M}-$ $\left.\mathrm{N}_{2}+\mathrm{H}^{+}\right)$: 182.0965; Found: 182.0970 .

1-(azidomethyl)-3-chlorobenzene (3h)

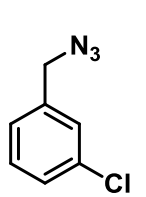

A vial containing $\mathrm{Cu}(\mathrm{dap})_{2} \mathrm{Cl} \quad(0.005$ equiv, $0.003 \mathrm{mmol}, \quad 2.20 \mathrm{mg}), \quad 1$-azido- $1 \lambda^{3}-$ benzo[ $d][1,2]$ iodaoxol-3(1H)-one $(1.00$ equiv, $0.50 \mathrm{mmol}, 145 \mathrm{mg})$ and equipped with a stirrer bar, sealed with a septum, was purged under a flux of nitrogen. Then degassed mixture of acetonitrile

$(2.5 \mathrm{~mL}$ ) and 1-chloro-3-methylbenzene (40.0 equiv, $20.0 \mathrm{mmol}, 2.40 \mathrm{~mL}$ ) was added and the tube was sealed and put one centimeter away from a $30 \mathrm{~W}$ fluorescent bulb and stirred for 16 hours. The crude reaction mixture was loaded directly on the chromatography column and purified with mixtures of hexane:DCM affording $3 \boldsymbol{h}$ as a colourless oil $(57.0 \mathrm{mg}, 68 \%)$. Data are in accordance with previous literature reports. ${ }^{19}{ }^{1} \mathrm{H}$ NMR (400 MHz, Chloroform- $d$ ) $\delta 7.33-7.32$ (m, 3H), 7.20 (m, 1H), 4.33 (s, 2H). ${ }^{13} \mathrm{C} \mathrm{NMR}(101 \mathrm{MHz}$, Chloroform-d) $\delta 137.5,134.8,130.3,128.6,128.3,126.3,54.2$. IR (neat film): 2927, 2100, 1600, 1576, 1476, 1431, 1339, 1257, 1079, 682. MS (EI): $167(\mathrm{M}), 139\left(\mathrm{M}-\mathrm{N}_{2}\right), 111\left(\mathrm{M}-\mathrm{CH}_{2} \mathrm{~N}_{3}\right)$. HRMS (APCI +): Calculated for $\mathrm{C}_{7} \mathrm{H}_{7} \mathrm{NCl}\left(\mathrm{M}-\mathrm{N}_{2}+\mathrm{H}^{+}\right)$: 140.0270; Found: 140.0267 .

1-(azidomethyl)-3-fluorobenzene (3i)

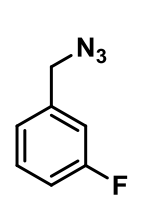

A vial containing $\mathrm{Cu}(\mathrm{dap})_{2} \mathrm{Cl} \quad(0.005$ equiv, $0.003 \mathrm{mmol}, \quad 2.20 \mathrm{mg}), \quad 1$-azido- $1 \lambda^{3}-$ benzo[ $d][1,2]$ iodaoxol-3(1H)-one (1.00 equiv, $0.50 \mathrm{mmol}, 145 \mathrm{mg}$ ) and equipped with a stirrer bar, sealed with a septum, was purged under a flux of nitrogen. Then degassed mixture of acetonitrile $(2.5 \mathrm{~mL}$ ) and 1-fluoro-3-methylbenzene ( 40.0 equiv, $20.0 \mathrm{mmol}, 2.20 \mathrm{~mL}$ ) was added and the tube was sealed and put one centimeter away from a $30 \mathrm{~W}$ fluorescent bulb and stirred for 16 hours. The crude reaction mixture was loaded directly on the chromatography column and purified with mixtures of hexane:DCM affording $3 \boldsymbol{i}$ as a colourless oil $(30.0 \mathrm{mg}, 39 \%)$. Data are in accordance with previous literature reports. ${ }^{11}{ }^{1} \mathrm{H}$ NMR (400 MHz, Chloroform- $d$ ) $\delta 7.36(\mathrm{~m}, 1 \mathrm{H}), 7.10(\mathrm{~m}, 1 \mathrm{H}), 7.06-7.01(\mathrm{~m}, 2 \mathrm{H}), 4.35(\mathrm{~s}, 2 \mathrm{H}) .{ }^{13} \mathrm{C}$ NMR $(101$ MHz, Chloroform- $d$ ) $\delta 163.1$ (d, $J=247.1 \mathrm{~Hz}), 138.0(\mathrm{~d}, J=7.3 \mathrm{~Hz}), 130.6(\mathrm{~d}, J=8.2 \mathrm{~Hz}), 123.8$ (d, $J=3.0$ $\mathrm{Hz}), 115.4(\mathrm{~d}, J=20.9 \mathrm{~Hz}), 115.2(\mathrm{~d}, J=21.6 \mathrm{~Hz}), 54.3(\mathrm{~d}, J=1.9 \mathrm{~Hz}) .{ }^{19} \mathrm{~F}$ NMR $(376 \mathrm{MHz}$, Chloroform- $d$ ) $\delta$ -112.30. IR (neat film): 2929, 2094, 1618, 1591, 1488, 1450, 1343, 1257, 1138, 781, 748, 685. MS (EI): 151 $(\mathrm{M}), 123\left(\mathrm{M}-\mathrm{N}_{2}\right), 109\left(\mathrm{M}-\mathrm{N}_{3}\right), 95\left(\mathrm{M}-\mathrm{CH}_{2} \mathrm{~N}_{3}\right)$. HRMS (APCI +): Calculated for $\mathrm{C}_{7} \mathrm{H}_{7} \mathrm{NF}\left(\mathrm{M}-\mathrm{N}_{2}+\mathrm{H}^{+}\right)$: 124.0560; Found: 124.0563. 
1-(azidomethyl)-4-nitrobenzene (3j)

$\mathrm{N}_{3}$ A vial containing $\mathrm{Cu}(\mathrm{dap})_{2} \mathrm{Cl} \quad(0.005$ equiv, $0.003 \mathrm{mmol}, 2.20 \mathrm{mg}), \quad$ 1-azido- $-1 \lambda^{3}-$
benzo[ $[d][1,2]$ iodaoxol- $-3(1 \mathrm{H})$-one $(1$ equiv, $0.50 \mathrm{mmol}, 145 \mathrm{mg}), 1-$ methyl-4-nitrobenzene $(40.0$
equiv, $20.0 \mathrm{mmol}, 2.75 \mathrm{~g})$ and equipped with a stirrer bar, sealed with a septum, was purged under a
$\mathrm{NO}_{2}$ flux of nitrogen. Then degassed acetonitrile $(2.5 \mathrm{~mL})$ was added and the tube was sealed and put 1.0 centimeter away from a $30 \mathrm{~W}$ fluorescent bulb and stirred for 16 hours. The crude reaction mixture was loaded directly on the chromatography column and purified with mixtures of hexane:EtOAc affording $\mathbf{3} \boldsymbol{j}$ as a colourless oil (45.0 mg, 50\%). Data are in accordance with previous literature reports. ${ }^{8}{ }^{1} \mathrm{H}$ NMR (400 MHz, Chloroformd) $\delta 8.27-8.23(\mathrm{~m}, 2 \mathrm{H}), 7.64-7.46(\mathrm{~m}, 2 \mathrm{H}), 4.51(\mathrm{~s}, 2 \mathrm{H}) .{ }^{13} \mathrm{C}$ NMR (101 MHz, Chloroform-d) $\delta$ 147.9, 142.8, 128.7, 124.2, 53.9. IR (neat film): 2961, 2922, 2852, 2100, 1607, 1519, 1342, 1258, 1015, 796. MS (EI): 150 (M $\left.-\mathrm{N}_{2}\right), 76\left(\mathrm{M}-\mathrm{NO}_{2}-\mathrm{CH}_{2} \mathrm{~N}_{3}\right)$. HRMS (APCI +): Calculated for $\mathrm{C}_{7} \mathrm{H}_{7} \mathrm{~N}_{2} \mathrm{O}_{2}\left(\mathrm{M}-\mathrm{N}_{2}+\mathrm{H}^{+}\right)$: 151.0502; Found: 151.0503 .

1-(azidomethyl)-4-methoxybenzene (3k)

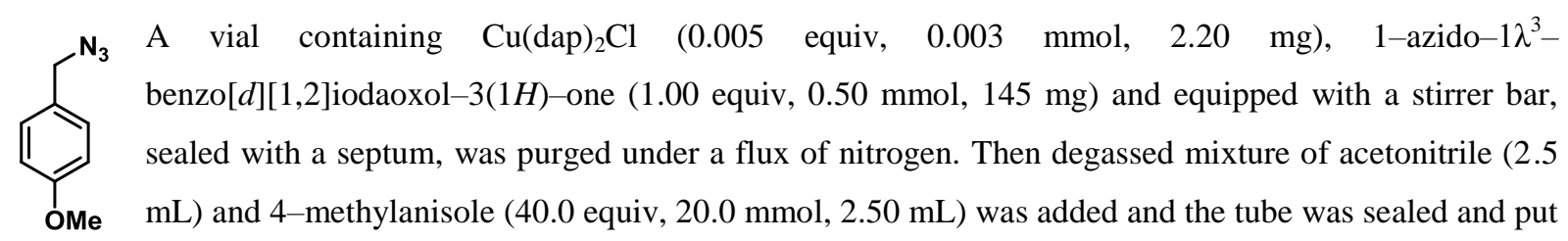
one centimeter away from a $30 \mathrm{~W}$ fluorescent bulb and stirred for 16 hours. The crude reaction mixture was loaded directly on the chromatography column and purified with mixtures of hexane:EtOAc affording $3 \boldsymbol{k}$ as a colourless oil (56.0 mg, 69\%). Data are in accordance with previous literature reports. ${ }^{8}{ }^{1} \mathrm{H}$ NMR $(400 \mathrm{MHz}$, Chloroform- $d$ ) $\delta$ 7.28-7.24 (m, 2H), 6.94-6.92 (m, 2H), 4.28 (s, 2H), $3.83(\mathrm{~s}, 3 \mathrm{H}) .{ }^{13} \mathrm{C}$ NMR $(101 \mathrm{MHz}$, Chloroform- $d$ ) $\delta 159.8,129.9,127.6,114.3,55.4,54.5$. IR (neat film): 2935, 2836, 2091, 1032, 1512, 1244, 1175, 1032, 812. MS (APCI +$)$ : $136\left(\mathrm{M}-\mathrm{N}_{2}+\mathrm{H}^{+}\right)$. HRMS (APCI +): Calculated for $\mathrm{C}_{8} \mathrm{H}_{10} \mathrm{ON}$ : 136.0757; Found: 136.0758 .

\section{4-(azidomethyl)benzonitrile ( $3 l$ )}

$\mathrm{N}_{3} \mathrm{~A}$ vial containing $\mathrm{Cu}(\mathrm{dap})_{2} \mathrm{Cl}(0.005$ equiv, $0.003 \mathrm{mmol}, 2.20 \mathrm{mg}), \quad$ 1-azido- $-1 \lambda^{3}-$
benzo[d][1,2]iodaoxol-3(1H)-one (1 equiv, $0.50 \mathrm{mmol}, 145 \mathrm{mg}), 4-$ methylbenzonitrile (40.0 equiv,
$20.0 \mathrm{mmol}, 2.45 \mathrm{~g})$ and equipped with a stirrer bar, sealed with a septum, was purged under a flux of
nitrogen. Then degassed acetonitrile $(2.5 \mathrm{~mL})$ was added and the tube was sealed and put 1.0 centimeter away from a $30 \mathrm{~W}$ fluorescent bulb and stirred for 16 hours. The crude reaction mixture was loaded directly on the chromatography column and purified with mixtures of hexane:DCM:MTBE affording $\mathbf{3 l}$ as a colourless oil (34.0 mg, 43\%). Data are in accordance with previous literature reports. ${ }^{9}{ }^{1} \mathrm{H}$ NMR $(500 \mathrm{MHz}$, Chloroform- $d$ ) $\delta 7.69(\mathrm{~d}, J=8.0 \mathrm{~Hz}, 2 \mathrm{H}), 7.44(\mathrm{~d}, J=8.0 \mathrm{~Hz}, 2 \mathrm{H}), 4.45(\mathrm{~s}, 2 \mathrm{H}) .{ }^{13} \mathrm{C}$ NMR $(126 \mathrm{MHz}$, Chloroform-d) $\delta 140.8,132.7,128.6,118.7,112.0,54.0$. IR (neat film): 2916, 2229, 2097, 1610, 1505, 1414, 1345, 1291, 847, 735. MS (APCI +): $159\left(\mathrm{M}+\mathrm{H}^{+}\right)$. HRMS (APCI + ): Calculated for $\mathrm{C}_{8} \mathrm{H}_{7} \mathrm{~N}_{4}\left(\mathrm{M}+\mathrm{H}^{+}\right)$: 159.06658; Found: 159.0665. 
1-(azidomethyl)-4-bromobenzene (3m)

$\mathrm{N}_{3} \mathrm{~A}$ vial containing $\mathrm{Cu}(\text { dap })_{2} \mathrm{Cl}(0.005$ equiv, $0.003 \mathrm{mmol}, 2.20 \mathrm{mg}), \quad 1-\mathrm{azido}-1 \lambda^{3}-$
benzo $[d][1,2]$ iodaoxol-3(1H)-one $(1.00$ equiv, $0.50 \mathrm{mmol}, 145 \mathrm{mg})$ and equipped with a stirrer bar,
sealed with a septum, was purged under a flux of nitrogen. Then degassed mixture of acetonitrile
$(2.5 \mathrm{~mL})$ and 1 -bromo-4-methylbenzene $(40.0$ equiv, $20.0 \mathrm{mmol}, 2.50 \mathrm{~mL})$ was added and the tube was sealed and put one centimeter away from a $30 \mathrm{~W}$ fluorescent bulb and stirred for 16 hours. The crude reaction mixture was loaded directly on the chromatography column and purified with mixtures of hexane:DCM affording $\mathbf{3 m}$ as a colourless oil (90.0 mg, 85\%). Data are in accordance with previous literature reports. ${ }^{10}{ }^{1} \mathrm{H}$ NMR $(400 \mathrm{MHz}$, Chloroform- $d) \delta 7.53-7.50(\mathrm{~m}, 2 \mathrm{H}), 7.21-7.19(\mathrm{~m}, 2 \mathrm{H}), 4.31(\mathrm{~s}, 2 \mathrm{H}) .{ }^{13} \mathrm{C}$ NMR (125 MHz, Chloroform-d) $\delta 134.5,132.1,129.9,122.5,54.2$. IR (neat film): 2926, 2093, 1488, 1405, 1282, 1246, 1070, 1012, 792, 672. MS (EI): $213\left({ }^{81} \mathrm{BrM}\right), 211\left({ }^{79} \mathrm{BrM}\right), 185\left({ }^{81} \mathrm{BrM}-\mathrm{N}_{2}\right), 183\left({ }^{79} \mathrm{BrM}-\mathrm{N}_{2}\right)$, $171\left({ }^{81} \mathrm{BrM}-\mathrm{N}_{3}\right), 169\left({ }^{79} \mathrm{BrM}-\mathrm{N}_{3}\right), 157\left({ }^{81} \mathrm{BrM}-\mathrm{CH}_{2} \mathrm{~N}_{3}\right), 155\left({ }^{79} \mathrm{BrM}-\mathrm{CH}_{2} \mathrm{~N}_{3}\right), 104\left({ }^{81} \mathrm{BrM}-\mathrm{Br}-\mathrm{N}_{2}\right), 76$ $\left(\mathrm{M}-\mathrm{Br}-\mathrm{CH}_{2} \mathrm{~N}_{3}\right)$. HRMS (APCI + ): Calculated for $\mathrm{C}_{7} \mathrm{H}_{7} \mathrm{NBr}\left(\mathrm{M}-\mathrm{N}_{2}+\mathrm{H}^{+}\right)$: 183.9756; Found: 183.9757 .

1-(azidomethyl)-4-chlorobenzene (3n)

$\mathrm{A}$ vial containing $\mathrm{Cu}(\mathrm{dap})_{2} \mathrm{Cl}(0.005$ equiv, $0.003 \mathrm{mmol}, 2.20 \mathrm{mg}), \quad 1-\mathrm{azido}-1 \lambda^{3}-$
benzo[d][1,2]iodaoxol-3(1H)-one $(1.00$ equiv, $0.50 \mathrm{mmol}, 145 \mathrm{mg})$ and equipped with a stirrer bar,
sealed with a septum, was purged under a flux of nitrogen. Then degassed mixture of acetonitrile
$(2.5 \mathrm{~mL})$ and $1-$ chloro-4-methylbenzene $(40.0$ equiv, $20.0 \mathrm{mmol}, 2.40 \mathrm{~mL})$ was added and the tube was sealed and put one centimeter away from a $30 \mathrm{~W}$ fluorescent bulb and stirred for 16 hours. The crude reaction mixture was loaded directly on the chromatography column and purified with mixtures of hexane:DCM affording $\mathbf{3 n}$ as a colourless oil (58.0 $\mathrm{mg}, 69 \%)$. Data are in accordance with previous literature reports. ${ }^{12}{ }^{1} \mathrm{H}$ NMR $\left(400 \mathrm{MHz}\right.$, Chloroform-d) $\delta 7.38-7.35(\mathrm{~m}, 2 \mathrm{H}), 7.28-7.25(\mathrm{~m}, 2 \mathrm{H}), 4.32\left(\mathrm{~s},{ }^{2 \mathrm{H}}\right) .{ }^{13} \mathrm{C}$ NMR (101 MHz, Chloroform-d) $\delta$ 134.3, 134.0, 129.6, 129.1, 54.2. IR (neat film): 2093, 1492, 1248, 1090, 796. MS (EI): $167(\mathrm{M}), 139\left(\mathrm{M}-\mathrm{N}_{2}\right), 111\left(\mathrm{M}-\mathrm{CH}_{2} \mathrm{~N}_{3}\right)$. HRMS (APCI +): Calculated for $\mathrm{C}_{7} \mathrm{H}_{7} \mathrm{NCl}\left(\mathrm{M}-\mathrm{N}_{2}\right.$ $\left.+\mathrm{H}^{+}\right):$140.0262; Found: 140.0262 .

1-(azidomethyl)-4-fluorobenzene (3o)

$\mathbf{N}_{3} \mathrm{~A}$ vial containing $\mathrm{Cu}(\text { dap })_{2} \mathrm{Cl} \quad(0.005$ equiv, $0.003 \mathrm{mmol}, \quad 2.20 \mathrm{mg}), \quad 1-$ azido-1 $\lambda^{3}-$ benzo[d][1,2]iodaoxol-3(1H)-one (1.00 equiv, $0.50 \mathrm{mmol}, 145 \mathrm{mg})$ and equipped with a stirrer bar, sealed with a septum, was purged under a flux of nitrogen. Then degassed mixture of acetonitrile $(2.5 \mathrm{~mL})$ and 1-fluoro-4-methylbenzene (40.0 equiv, $20.0 \mathrm{mmol}, 2.20 \mathrm{~mL})$ was added and the tube was sealed and put one centimeter away from a $30 \mathrm{~W}$ fluorescent bulb and stirred for 16 hours. The crude reaction mixture was loaded directly on the chromatography column and purified with mixtures of hexane:DCM affording $3 o$ as a colourless oil (34.0 mg, 51\%). Data are in accordance with previous literature reports. ${ }^{11}{ }^{1} \mathrm{H}$ NMR (400 MHz, Chloroform-d) $\delta 7.32-7.28(\mathrm{~m}, 2 \mathrm{H}), 7.10-7.05(\mathrm{~m}, 2 \mathrm{H}), 4.32(\mathrm{~s}, 2 \mathrm{H}) .{ }^{13} \mathrm{C}$ NMR (101 MHz, Chloroform-d) $\delta 162.8(\mathrm{~d}, J=247.1 \mathrm{~Hz}), 131.3(\mathrm{~d}, J=3.3 \mathrm{~Hz}), 130.2(\mathrm{~d}, J=8.3 \mathrm{~Hz}), 116.0$ $(\mathrm{d}, J=21.6 \mathrm{~Hz}), 54.2 .{ }^{19} \mathrm{~F}$ NMR (376 MHz, Chloroform-d) $\delta-113.5$. IR (neat film): 2931, 2093, 1602, 1509, 1222, 820. MS (EI): $151(\mathrm{M}), 123\left(\mathrm{M}-\mathrm{N}_{2}\right), 109\left(\mathrm{M}-\mathrm{N}_{3}\right), 95\left(\mathrm{M}-\mathrm{CH}_{2} \mathrm{~N}_{3}\right)$. HRMS (APCI +): Calculated for $\mathrm{C}_{7} \mathrm{H}_{7} \mathrm{NF}\left(\mathrm{M}-\mathrm{N}_{2}+\mathrm{H}^{+}\right)$: 124.0567 ; Found: 124.0563 . 
1-(4-(azidomethyl)phenyl)ethan-1-one (3p)

$\mathrm{N}_{3} \quad$ A vial containing $\mathrm{Cu}(\mathrm{dap})_{2} \mathrm{Cl} \quad(0.005$ equiv, $0.003 \mathrm{mmol}, \quad 2.20 \mathrm{mg}), \quad 1$-azido- $1 \lambda^{3}-$ benzo[d][1,2]iodaoxol-3(1H)-one (1.00 equiv, $0.50 \mathrm{mmol}, 145 \mathrm{mg}$ ) and equipped with a stirrer bar, sealed with a septum, was purged under a flux of nitrogen. Then degassed mixture of acetonitrile (2.5 $\mathrm{mL}$ ) and methyl $p$-tolyl ketone ( 40.0 equiv, $20.0 \mathrm{mmol}, 2.70 \mathrm{~mL}$ ) was added and the tube was sealed and put one centimeter away from a $30 \mathrm{~W}$ fluorescent bulb and stirred for 16 hours. The crude reaction mixture was loaded directly on the chromatography column and purified with mixtures of hexane:EtOAc affording $3 \boldsymbol{p}$ as a colourless oil $(31.0 \mathrm{mg}, 35 \%)$. Data are in accordance with previous literature reports. ${ }^{13}{ }^{1} \mathrm{H}$ NMR (500 MHz, Chloroform-d) $\delta$ 7.98-7.96 (m, 2H), 7.42-7.40 (m, 2H), 4.42 (s, 2H), 2.61 (s, $3 \mathrm{H}) .{ }^{13} \mathrm{C}$ NMR (126 MHz, Chloroform-d) $\delta$ 197.6, 140.7, 137.1, 129.0, 128.3, 54.4, 26.8. IR (neat film): 2925, 2097, 1682, 1608, 1358, 1264, 957, 813, 594. MS (APCI +): $148\left(\mathrm{M}-\mathrm{N}_{2}+\mathrm{H}^{+}\right)$. HRMS (APCI +): Calculated for $\mathrm{C}_{9} \mathrm{H}_{10} \mathrm{NO}\left(\mathrm{M}-\mathrm{N}_{2}+\mathrm{H}^{+}\right)$: 148.0757; Found: 1480.0752 .

methyl 4-(azidomethyl)benzoate (3q)

$\mathrm{N}_{3} \quad$ A vial containing $\mathrm{Cu}(\text { dap })_{2} \mathrm{Cl} \quad(0.005$ equiv, $0.003 \quad \mathrm{mmol}, \quad 2.20 \mathrm{mg}), \quad 1$-azido-1 $\lambda^{3}-$ benzo[ $d][1,2]$ iodaoxol-3( $1 \mathrm{H})$-one ( 1 equiv, $0.50 \mathrm{mmol}, 145 \mathrm{mg}$ ), methyl $p$-toluate (40.0 equiv, 20.0 $\mathrm{mmol}, 3.00 \mathrm{~g}$ ) and equipped with a stirrer bar, sealed with a septum, was purged under a flux of - nitrogen. Then degassed acetonitrile $(2.5 \mathrm{~mL})$ was added and the tube was sealed and put 1.0
centimeter away from a $30 \mathrm{~W}$ fluorescent bulb and stirred for 16 hours. The crude reaction mixture was loaded directly on the chromatography column and purified with mixtures of hexane:MTBE affording $\mathbf{3 q}$ as a colourless oil (48.0 mg, 50\%). Data are in accordance with previous literature reports. ${ }^{14} \mathrm{H}$ NMR (500 MHz, Chloroform-d) $\delta 8.07-8.04(\mathrm{~m}, 2 \mathrm{H}), 7.41-7.38(\mathrm{~m}, 2 \mathrm{H}), 4.42(\mathrm{~s}, 2 \mathrm{H}), 3.93(\mathrm{~s}, 3 \mathrm{H}) .{ }^{13} \mathrm{C}$ NMR $(126 \mathrm{MHz}$, Chloroform- $d$ ) $\delta 166.8,140.5,130.3,130.1,128.1,54.4,52.4$. IR (neat film): 3057, 2090, 1434, 1246, 1120, 828, 745, 725. MS (EI): $163\left(\mathrm{M}-\mathrm{N}_{2}\right), 132\left(\mathrm{M}-\mathrm{CO}_{2} \mathrm{Me}\right), 104\left(\mathrm{M}-\mathrm{CO}_{2} \mathrm{Me}-\mathrm{N}_{2}\right), 76\left(\mathrm{M}-\mathrm{CO}_{2} \mathrm{Me}-\mathrm{CH}_{2} \mathrm{~N}_{3}\right)$. HRMS (APCI +): Calculated for $\mathrm{C}_{9} \mathrm{H}_{10} \mathrm{NO}_{2}\left(\mathrm{M}-\mathrm{N}_{2}\right)$ : 164.0706; Found: 164.0706.

1-(azidomethyl)-3-bromo-5-methylbenzene (3r)

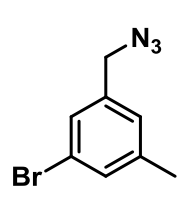

A vial containing $\mathrm{Cu}(\mathrm{dap})_{2} \mathrm{Cl}(0.005$ equiv, $0.003 \mathrm{mmol}, 2.20 \mathrm{mg}), 1$-azido- $1 \lambda^{3}-$ benzo[ $d][1,2]$ iodaoxol-3(1H)-one (1.00 equiv, $0.50 \mathrm{mmol}, 145 \mathrm{mg})$ and equipped with a stirrer bar, sealed with a septum, was purged under a flux of nitrogen. Then degassed mixture of acetonitrile $(2.5 \mathrm{~mL}$ ) and 1-bromo-3,5-dimethylbenzene (40.0 equiv, $20.0 \mathrm{mmol}, 2.50 \mathrm{~mL}$ ) was added and the tube was sealed and put one centimeter away from a $30 \mathrm{~W}$ fluorescent bulb and stirred for 16 hours. The crude reaction mixture was loaded directly on the chromatography column and purified with mixtures of hexane:DCM affording $3 \boldsymbol{r}$ as a colourless oil (54.0 mg, 48\%). ${ }^{1} \mathrm{H}$ NMR (400 MHz, Chloroformd) $\delta 7.31(\mathrm{~m}, 1 \mathrm{H}), 7.27(\mathrm{~m}, 1 \mathrm{H}), 7.05(\mathrm{~m}, 1 \mathrm{H}), 4.28(\mathrm{~s}, 2 \mathrm{H}), 2.35(\mathrm{~d}, J=0.8 \mathrm{~Hz}, 3 \mathrm{H}) .{ }^{13} \mathrm{C} \mathrm{NMR}(101 \mathrm{MHz}$, Chloroform- $d$ ) $\delta 140.9,137.5,132.1,128.3,127.6,122.7,54.2,21.3$. IR (neat film): 2919, 2092, 1604, 1573, 1444, 1337, 1278, 1253, 845, 812, 679. MS (EI): $227\left({ }^{81} \mathrm{BrM}\right), 225\left({ }^{79} \mathrm{BrM}\right), 198\left({ }^{81} \mathrm{BrM}-\mathrm{N}_{2}\right), 196\left({ }^{79} \mathrm{BrM}-\right.$ $\left.\mathrm{N}_{2}\right), 183\left(\mathrm{M}-\mathrm{N}_{3}\right), 169\left(\mathrm{M}-\mathrm{CH}_{2} \mathrm{~N}_{3}\right)$. HRMS (EI): Calculated for $\mathrm{C}_{8} \mathrm{H}_{8} \mathrm{~N}_{3} \mathrm{Br}$ : 224.9896; Found: 224.9893. 
1-(azidomethyl)-2,4,5-trimethylbenzene (3s)

(N)

A vial containing $\mathrm{Cu}(\mathrm{dap})_{2} \mathrm{Cl} \quad(0.005$ equiv, $0.003 \mathrm{mmol}, \quad 2.20 \mathrm{mg}), \quad 1$-azido- $1 \lambda^{3}-$ benzo[d][1,2]iodaoxol-3(1H)-one (1 equiv, $0.50 \mathrm{mmol}, 145 \mathrm{mg}), 1,2,4,5$-tetramethylbenzene (40.0 equiv, $20.0 \mathrm{mmol}, 2.70 \mathrm{~g}$ ) and equipped with a stirrer bar, sealed with a septum, was purged under a flux of nitrogen. Then degassed acetonitrile $(2.5 \mathrm{~mL})$ was added and the tube was sealed and put 1.0 centimeter away from a $30 \mathrm{~W}$ fluorescent bulb and stirred for 16 hours. The crude reaction mixture was loaded directly on the chromatography column and purified with mixtures of hexane:DCM affording $3 \boldsymbol{s}$ as a colourless oil (68.0 mg, 78\%). ${ }^{1} \mathrm{H}$ NMR (400 MHz, Chloroform- $d$ ) $\delta 7.02(\mathrm{~s}, 1 \mathrm{H}), 7.00(\mathrm{~s}, 1 \mathrm{H}), 4.29(\mathrm{~s}, 2 \mathrm{H})$, $2.30(\mathrm{~s}, 3 \mathrm{H}), 2.24$ (s, 3H), 2.24 (s, 3H). ${ }^{13} \mathrm{C}$ NMR (101 MHz, Chloroform-d) $\delta$ 137.1, 134.3, 134.1, 132.2, 131.0, 130.7, 53.0, 19.5, 19.3, 18.5. IR (neat film): 2921, 2089, 1507, 1456, 1237, 863. MS (EI): 175 (M), 133 (M $\left.\mathrm{N}_{3}\right)$. HRMS (APCI +): Calculated for $\mathrm{C}_{10} \mathrm{H}_{14} \mathrm{~N}\left(\mathrm{M}-\mathrm{N}_{2}+\mathrm{H}^{+}\right)$: 148.1121 ; Found: 148.1118 .

1-(azidomethyl)-3,5-dimethylbenzene (3t)

$\mathrm{N}_{3} \mathrm{~A}$ vial containing $\mathrm{Cu}(\mathrm{dap})_{2} \mathrm{Cl} \quad(0.005$ equiv, $0.003 \mathrm{mmol}, 2.20 \mathrm{mg}), \quad 1$-azido- $-1 \lambda^{3}-$
benzo[d][1,2]iodaoxol-3(1H)-one (1.00 equiv, $0.50 \mathrm{mmol}, 145 \mathrm{mg})$ and equipped with a stirrer bar,
sealed with a septum, was purged under a flux of nitrogen. Then degassed mixture of acetonitrile $(2.5 \mathrm{~mL})$ and mesitylene (40.0 equiv, $20.0 \mathrm{mmol}, 2.80 \mathrm{~mL}$ ) was added and the tube was sealed and put one centimeter away from a $30 \mathrm{~W}$ fluorescent bulb and stirred for 16 hours. The crude reaction mixture was loaded directly on the chromatography column and purified with mixtures of hexane:DCM affording $3 t$ as a colourless oil $(53.0 \mathrm{mg}, 60 \%)$. Data are in accordance with previous literature reports. ${ }^{71} \mathrm{H}$ NMR (400 MHz, Chloroform- $d$ ) $\delta 6.99$ (s, 1H), 6.94 (s, 2H), 4.27 (s, 2H), 2.34 (s, 6H). ${ }^{13} \mathrm{C} \mathrm{NMR} \mathrm{(101} \mathrm{MHz,} \mathrm{Chloroform-d)} \mathrm{\delta 138.6,} \mathrm{135.3,}$ 130.1, 126.2, 55.0, 21.4. IR (neat film): 2919, 2092, 1607, 1243, 840. MS (EI): 161 (M), 133 (M - N N $_{2}, 119$ (M$\mathrm{N}_{3}$ ). HRMS (EI): Calculated for $\mathrm{C}_{9} \mathrm{H}_{11} \mathrm{~N}_{3}$ (M): 161.0947; Found: 161.0944 .

(azidomethylene)dibenzene $(3 \boldsymbol{u})$<smiles>NC(c1ccccc1)c1ccccc1</smiles>

A vial containing $\mathrm{Cu}(\mathrm{dap})_{2} \mathrm{Cl}(0.005$ equiv, $0.003 \mathrm{mmol}, 2.20 \mathrm{mg}), 1$-azido- $1 \lambda^{3}-$ benzo[d][1,2]iodaoxol-3(1H)-one (1.00 equiv, $0.50 \mathrm{mmol}, 145 \mathrm{mg})$ and equipped with a stirrer bar, sealed with a septum, was purged under a flux of nitrogen. Then degassed mixture of acetonitrile $(2.5 \mathrm{~mL})$ and diphenylmethane (40.0 equiv, $20.0 \mathrm{mmol}, 5.85 \mathrm{~mL})$ was added and the tube was sealed and put one centimeter away from a $30 \mathrm{~W}$ fluorescent bulb and stirred for 16 hours. The crude reaction mixture was loaded directly on the chromatography column and purified with mixtures of hexane:EtOAc affording $3 \boldsymbol{u}$ as a colourless oil $(69.0 \mathrm{mg}, 66 \%)$. Data are in accordance with previous literature reports. ${ }^{11}{ }^{1} \mathrm{H}$ NMR (400 MHz, Chloroform-d) $\delta 7.40-7.29$ (m, 10H), 5.73 (s, 1H). ${ }^{13} \mathrm{C}$ NMR (101 MHz, Chloroform-d) $\delta 139.7,128.8,128.2,127.5$, 68.6. IR (neat film): 3029, 2094, 1601, 1493, 1452, 1238 , 1079, 1030, 909, 870, 741, 695. MS (EI): $181\left(\mathrm{M}-\mathrm{N}_{2}\right), 104\left(\mathrm{M}-\mathrm{Ph}-\mathrm{N}_{2}\right), 77\left(\mathrm{M}-\mathrm{PhCHN}_{3}\right)$. HRMS $\left(\right.$ APCI +): Calculated for $\mathrm{C}_{13} \mathrm{H}_{12} \mathrm{~N}\left(\mathrm{M}-\mathrm{N}_{2}+\mathrm{H}^{+}\right)$: 182.0964; Found: 182.0961 . 
(1-azidoethyl)benzene $(3 v)$

$Y^{N_{3}}$

A vial containing $\mathrm{Cu}(\text { dap })_{2} \mathrm{Cl} \quad(0.005$ equiv, $0.003 \quad \mathrm{mmol}, \quad 2.20 \mathrm{mg}), \quad 1$-azido-1 $\lambda^{3}-$ benzo[d][1,2]iodaoxol-3(1H)-one (1.00 equiv, $0.50 \mathrm{mmol}, 145 \mathrm{mg})$ and equipped with a stirrer bar, sealed with a septum, was purged under a flux of nitrogen. Then degassed mixture of acetonitrile (2.5 $\mathrm{mL}$ ) and ethylbenzene (40.0 equiv, $20.0 \mathrm{mmol}, 2.45 \mathrm{~mL}$ ) was added and the tube was sealed and put one centimeter away from a $30 \mathrm{~W}$ fluorescent bulb and stirred for 16 hours. The crude reaction mixture was loaded directly on the chromatography column and purified with mixtures of hexane:DCM affording $3 v$ as a colourless oil $(46.0 \mathrm{mg}, 62 \%)$. Data are in accordance with previous literature reports. ${ }^{20}{ }^{1} \mathrm{H} \mathrm{NMR}(400 \mathrm{MHz}$, Chloroform-d) $\delta 7.42-7.31(\mathrm{~m}, 5 \mathrm{H}), 4.63(\mathrm{q}, J=6.8 \mathrm{~Hz}, 1 \mathrm{H}), 1.54(\mathrm{~d}, J=6.8 \mathrm{~Hz}, 1 \mathrm{H}) .{ }^{13} \mathrm{C} \mathrm{NMR}(101 \mathrm{MHz}$, Chloroform-d) $\delta 141.1,129.0,128.4,126.6,61.3,21.8$. IR (neat film): 3031, 2980, 2086, 1493, 1453, 1376, 1305, 1243, 1062, 1028, 1005, 759, 697. MS (EI): $147(\mathrm{M}), 119\left(\mathrm{M}-\mathrm{N}_{2}\right), 105\left(\mathrm{M}-\mathrm{N}_{3}\right), 77\left(\mathrm{M}-\mathrm{CH}_{3} \mathrm{CHN}_{3}\right)$. HRMS (APCI +): Calculated for $\mathrm{C}_{8} \mathrm{H}_{10} \mathrm{~N}\left(\mathrm{M}-\mathrm{N}_{2}+\mathrm{H}^{+}\right)$: 120.0808 ; Found: 120.0811 .

1-(1-azidoethyl)-4-ethylbenzene (3w)

$\mathrm{N}_{3} \mathrm{~A}$ vial containing $\mathrm{Cu}(\text { dap })_{2} \mathrm{Cl} \quad(0.005$ equiv, $0.003 \mathrm{mmol}, 2.20 \mathrm{mg}), \quad 1-$ azido-1 $\lambda^{3}-$ benzo[d][1,2]iodaoxol-3(1H)-one (1.00 equiv, $0.50 \mathrm{mmol}, 145 \mathrm{mg})$ and equipped with a stirrer bar, sealed with a septum, was purged under a flux of nitrogen. Then degassed mixture of acetonitrile (2.5 $\mathrm{mL}$ ) and 1,4-diethylbenzene (40.0 equiv, $20.0 \mathrm{mmol}, 3.10 \mathrm{~mL}$ ) was added and the tube was sealed and put one centimeter away from a $30 \mathrm{~W}$ fluorescent bulb and stirred for 16 hours. The crude reaction mixture was loaded directly on the chromatography column and purified with mixtures of hexane:DCM affording $3 w$ as a colourless oil $(72.0 \mathrm{mg}, 82 \%) .{ }^{1} \mathrm{H}$ NMR $(400 \mathrm{MHz}$, Chloroform- $d) \delta 7.26(\mathrm{dd}, J=8.2 \mathrm{~Hz}, 2 \mathrm{H}), 7.22(\mathrm{~d}, J=$ $8.2 \mathrm{~Hz}, 2 \mathrm{H}), 4.59(\mathrm{q}, J=6.7 \mathrm{~Hz}, 1 \mathrm{H}), 2.66(\mathrm{q}, J=7.6 \mathrm{~Hz}, 2 \mathrm{H}), 1.53(\mathrm{~d}, J=6.8 \mathrm{~Hz}, 3 \mathrm{H}), 1.25(\mathrm{t}, J=7.6 \mathrm{~Hz}, 3 \mathrm{H})$. ${ }^{13} \mathrm{C}$ NMR (101 MHz, Chloroform- $\left.d\right) \delta 144.4,138.2,128.4,126.5,61.1,28.7,21.7,15.6$. IR (neat film): 2967 , 2932, 2873, 2089, 1514, 1453, 1241, 1062, 992, 830, 633. MS (EI): $175(\mathrm{M}), 147\left(\mathrm{M}-\mathrm{N}_{2}\right), 133\left(\mathrm{M}-\mathrm{N}_{3}\right), 105$ $\left(\mathrm{M}-\mathrm{CH}_{3} \mathrm{CHN}_{3}\right), 90\left(\mathrm{M}-\mathrm{CH}_{3} \mathrm{CHN}_{3}-\mathrm{CH}_{3}\right), 76\left(\mathrm{M}-\mathrm{CH}_{3} \mathrm{CHN}_{3}-\mathrm{CH}_{2} \mathrm{CH}_{3}\right)$. HRMS (APCI +): Calculated for $\mathrm{C}_{10} \mathrm{H}_{14} \mathrm{~N}\left(\mathrm{M}-\mathrm{N}_{2}+\mathrm{H}^{+}\right)$: 148.1121; Found: 148.1120 .

1-(2-azidopropan-2-yl)-3,5-diisopropylbenzene (3x)

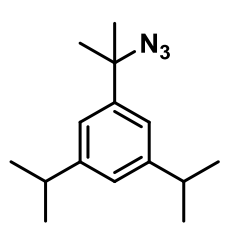

A vial containing $\mathrm{Cu}(\text { dap })_{2} \mathrm{Cl}(0.005$ equiv, $0.003 \mathrm{mmol}, 2.20 \mathrm{mg}), \quad 1$-azido-1 $\lambda^{3}-$ benzo $[d][1,2]$ iodaoxol-3(1H)-one $(1.00$ equiv, $0.50 \mathrm{mmol}, 145 \mathrm{mg})$ and equipped with a stirrer bar, sealed with a septum, was purged under a flux of nitrogen. Then degassed mixture of acetonitrile $(2.5 \mathrm{~mL})$ and 1,3,5-triisopropylbenzene (40.0 equiv, $20.0 \mathrm{mmol}, 4.80 \mathrm{~mL}$ ) was added and the tube was sealed and put one centimeter away from a $30 \mathrm{~W}$ fluorescent bulb and stirred for 16 hours. The crude reaction mixture was loaded directly on the chromatography column and purified with mixtures of hexane:EtOAc affording $3 \boldsymbol{x}$ as a colourless oil $(96.0 \mathrm{mg}, 78 \%)$. ${ }^{1} \mathrm{H}$ NMR (400 MHz, Chloroform- $d$ ) $\delta 7.18(\mathrm{~d}, J=1.6 \mathrm{~Hz}, 2 \mathrm{H}), 6.99(\mathrm{~m}, 1 \mathrm{H}), 2.91$ (quin., $J=6.9 \mathrm{~Hz}, 2 \mathrm{H}), 1.59(\mathrm{~s}, 6 \mathrm{H}), 1.27$ (dd, $J=6.9,0.8 \mathrm{~Hz}$, $12 \mathrm{H}) .{ }^{13} \mathrm{C}$ NMR $(101 \mathrm{MHz}$, Chloroform-d) $\delta 149.1,148.9,123.1,120.2,72.9,34.5,31.9,24.3$. IR (neat film):

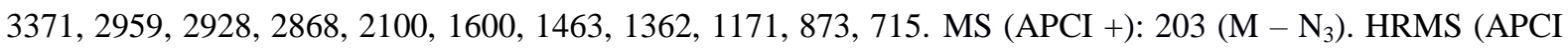
+): Calculated for $\mathrm{C}_{15} \mathrm{H}_{23}\left(\mathrm{M}-\mathrm{N}_{3}\right)$ : 203.1790; Found: 203.1800 . 
1-(2-azidopropan-2-yl)-4-methylbenzene, 1-(azidomethyl)-4-isopropylbenzene (3y)

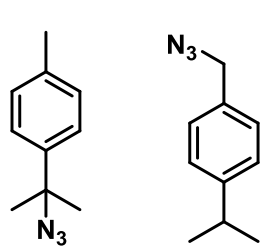

A vial containing $\mathrm{Cu}(\mathrm{dap})_{2} \mathrm{Cl}(0.005$ equiv, $0.003 \mathrm{mmol}, 2.20 \mathrm{mg}), 1$-azido- $1 \lambda^{3}-$ benzo[d][1,2]iodaoxol-3(1H)-one (1.00 equiv, $0.50 \mathrm{mmol}, 145 \mathrm{mg})$ and equipped with a stirrer bar, sealed with a septum, was purged under a flux of nitrogen. Then degassed mixture of acetonitrile $(2.5 \mathrm{~mL})$ and 4-isopropyltoluene (40.0 equiv, $20.0 \mathrm{mmol}, 3.10 \mathrm{~mL}$ ) was added and the tube was sealed and put one centimeter away from a $30 \mathrm{~W}$ fluorescent

bulb and stirred for 16 hours. The crude reaction mixture was loaded directly on the chromatography column and purified with mixtures of hexane:DCM affording $\mathbf{3 y}$ as a colourless oil, as a mixture of 2 unseparable regioisomers (66.0 mg, 75\% of a 10:1 271az1: 271az2). Data are in accordance with previous literature reports. ${ }^{21}$ 271az1: ${ }^{1} \mathrm{H}$ NMR (500 MHz, Chloroform-d) $\delta$ 7.37-7.34 (m, 2H), 7.21-7.19 (m, 2H), 2.37 (s, 3H), $1.64(\mathrm{~s}, 6 \mathrm{H}) .{ }^{13} \mathrm{C}$ NMR $\left(126 \mathrm{MHz}\right.$, Chloroform-d) $\delta 141.8,137.2,129.3,125.2,63.8,28.5,21.1 .271 a z 2:{ }^{1} \mathrm{H}$ NMR (500 MHz, Chloroform-d) $\delta 7.26$ (s, 4H), 4.32 (s, 2H), 2.94 (hept., $J=6.7$ Hz, 1H), 1.28 (d, $J=6.9 \mathrm{~Hz}$, $6 \mathrm{H}) .{ }^{13} \mathrm{C}$ NMR (126 MHz, Chloroform-d) $\delta$ 149.2, 132.9, 128.4, 127.0, 54.8, 34.0, 24.1. IR (neat film): 2977 , 2100, 1514, 1366, 1254, 1145, 1099, 815, 722, 617. MS (EI): $147\left(\mathrm{M}-\mathrm{N}_{2}\right), 133\left(\mathrm{M}-\mathrm{N}_{3}\right), 118\left(\mathrm{M}-\mathrm{N}_{3}-\mathrm{CH}_{3}\right)$, $106\left(\mathrm{M}-\mathrm{N}_{3}-2 \mathrm{CH}_{3}\right), 91\left(\mathrm{M}-\mathrm{N}_{3}-\mathrm{C}\left(\mathrm{CH}_{3}\right)_{2} \mathrm{~N}_{3}\right)$. HRMS (APCI +): Calculated for $\mathrm{C}_{10} \mathrm{H}_{14} \mathrm{~N}\left(\mathrm{M}-\mathrm{N}_{2}+\mathrm{H}^{+}\right)$: 148.1121; Found: 148.1118.

tert-butyl 2-(azidomethyl)-1H-indole-1-carboxylate (3aa)

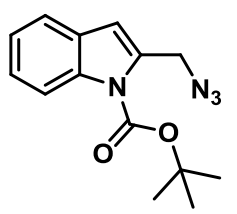
A vial containing $\mathrm{Cu}(\mathrm{dap})_{2} \mathrm{Cl}(0.005$ equiv, $0.003 \mathrm{mmol}, 2.20 \mathrm{mg}), 1$-azido-1 $\lambda^{3}-$ benzo[ $d][1,2]$ iodaoxol-3(1H)-one (1 equiv, $0.50 \mathrm{mmol}, 145 \mathrm{mg}$ ), tert-butyl 2-methyl-1Hindole-1-carboxylate (40.0 equiv, $20.0 \mathrm{mmol}, 2.30 \mathrm{~g}$ ) and equipped with a stirrer bar, sealed with a septum, was purged under a flux of nitrogen. Then degassed acetonitrile (2.5 $\mathrm{mL}$ ) was added and the tube was sealed and put 1.0 centimeter away from a $30 \mathrm{~W}$ fluorescent bulb and stirred for 16 hours. The crude reaction mixture was loaded directly on the chromatography column and purified with mixtures of hexane:toluene affording 3aa as a colourless oil (45.0 mg, 66\%). ${ }^{1} \mathrm{H} \mathrm{NMR} \mathrm{(400} \mathrm{MHz}$, Chloroform-d) $\delta 8.11(\mathrm{~d}, J=8.2,1 \mathrm{H}), 7.54(\mathrm{~d}, J=7.6 \mathrm{~Hz}, 1 \mathrm{H}), 7.32(\mathrm{~m}, 1 \mathrm{H}), 7.24,(\operatorname{app~t}, \mathrm{H}=7.3 \mathrm{~Hz}, 1 \mathrm{H})$, $6.67(\mathrm{~d}, J=0.6 \mathrm{~Hz}, 1 \mathrm{H}), 4.75(\mathrm{~s}, 2 \mathrm{H}), 1.71(\mathrm{~s}, 9 \mathrm{H}) .{ }^{13} \mathrm{C} \mathrm{NMR}(101 \mathrm{MHz}$, Chloroform-d) $\delta$ 150.3, 136.9, 134.8, 128.6, 124.8, 123.2, 120.9, 115.9, 110.4, 84.8, 49.7, 28.3. IR (neat film): 2978, 2094, 1730, 1452, 1369, 1323, 1155, 1117, 1085, 744. MS (APCI +): $189\left(\mathrm{M}-\mathrm{N}_{2}-\mathrm{C}\left(\mathrm{CH}_{3}\right)_{3}+2 \mathrm{H}^{+}\right), 174\left(\mathrm{M}-\mathrm{N}_{3}-\mathrm{C}\left(\mathrm{CH}_{3}\right)_{3}+\right.$ $\left.\mathrm{H}^{+}\right), 145\left(\mathrm{M}-\mathrm{N}_{2}-\mathrm{COOC}\left(\mathrm{CH}_{3}\right)_{3}+2 \mathrm{H}^{+}\right), 130\left(\mathrm{M}-\mathrm{N}_{3}-\mathrm{COOC}\left(\mathrm{CH}_{3}\right)_{3}+\mathrm{H}^{+}\right)$. HRMS (APCI +$)$: Calculated for $\mathrm{C}_{10} \mathrm{H}_{8} \mathrm{O}_{2} \mathrm{~N}$ : 174.0550; From: 174.0555.

2-(azidomethyl)benzo[b]thiophene $(\mathbf{3} \boldsymbol{b} \boldsymbol{b})$

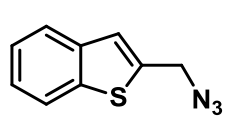

A vial containing $\mathrm{Cu}(\mathrm{dap}){ }_{2} \mathrm{Cl}(0.005$ equiv, $0.003 \mathrm{mmol}, 2.20 \mathrm{mg}), 1$-azido- $1 \lambda^{3}-$ benzo[d][1,2]iodaoxol-3(1H)-one $\quad(1 \quad$ equiv, $0.50 \quad \mathrm{mmol}, \quad 145 \quad \mathrm{mg}), \quad 2-$ methylbenzo[b]thiophene (40.0 equiv, $20.0 \mathrm{mmol}, 2.95 \mathrm{~g}$ ) and equipped with a stirrer bar, sealed with a septum, was purged under a flux of nitrogen. Then degassed acetonitrile $(2.5 \mathrm{~mL})$ was added and the tube was sealed and put 1.0 centimeter away from a $30 \mathrm{~W}$ fluorescent bulb and stirred for 16 hours. The crude reaction mixture was loaded directly on the chromatography column and purified with mixtures of 
hexane:DCM affording $3 \boldsymbol{b} \boldsymbol{b}$ as a colourless oil $(87.0 \mathrm{mg}$, 92\%). Data are in accordance with previous literature reports. ${ }^{14} \mathrm{H}$ NMR $(400 \mathrm{MHz}$, Chloroform- $d) \delta 7.83(\mathrm{~m}, 1 \mathrm{H}), 7.77(\mathrm{~m}, 1 \mathrm{H}), 7.40-7.33(\mathrm{~m}, 2 \mathrm{H})$, 7.27 (m, 1H), 4.59 (s, 2H). ${ }^{13} \mathrm{C}$ NMR (101 MHz, Chloroform- $d$ ) $\delta$ 140.3, 139.4, 138.4, 124.9, 124.7, 123.9, 123.8, 122.6, 50.1. IR (neat film): 3057, 2090, 1434, 1246, 1120, 828, 745, 725. MS (APCI +): $162\left(\mathrm{M}-\mathrm{N}_{2}+\right.$ $\mathrm{H}^{+}$). HRMS (APCI +): Calculated for $\mathrm{C}_{9} \mathrm{H}_{7} \mathrm{NS}\left(\mathrm{M}-\mathrm{N}_{2}+\mathrm{H}^{+}\right)$: 162.0372; Found: 162.0371 .

(azidomethyl)(p-tolyl)sulfane (3cc)

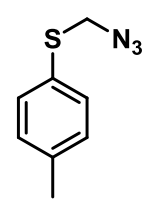

A vial containing $\mathrm{Cu}(\text { dap })_{2} \mathrm{Cl} \quad(0.005$ equiv, $0.003 \mathrm{mmol}, \quad 2.20 \mathrm{mg}), \quad 1$-azido- $1 \lambda^{3}-$ benzo[ $d][1,2]$ iodaoxol-3(1H)-one (1.00 equiv, $0.50 \mathrm{mmol}, 145 \mathrm{mg}$ ) and equipped with a stirrer bar, sealed with a septum, was purged under a flux of nitrogen. Then degassed mixture of acetonitrile $(2.5 \mathrm{~mL}$ ) and methyl $p$-tolyl sulfide (40.0 equiv, $20.0 \mathrm{mmol}, 2.70 \mathrm{~mL}$ ) was added and the tube was sealed and put one centimeter away from a $30 \mathrm{~W}$ fluorescent bulb and stirred for 16 hours. The crude reaction mixture was loaded directly on the chromatography column and purified with mixtures of hexane:DCM affording $3 \boldsymbol{c c}$ as a colourless oil (45.0 mg, 50\%). ${ }^{1} \mathrm{H}$ NMR (500 MHz, Chloroform- $d$ ) $\delta 7.42-7.41$ (m, 2H), 7.17-7.16 (m, 2H), 4.49 (s, 2H), 2.36 (s, 3H). ${ }^{13} \mathrm{C}$ NMR (126 MHz, Chloroform-d) $\delta 138.3,132.1$, 130.2, 129.8, 56.7, 21.3. IR (neat film): 2921, 2097, 1493, 1222, 1197, 864, 804. MS (EI): 151 (M - N 2 ), 123 $\left(\mathrm{M}-\mathrm{CH}_{2} \mathrm{~N}_{3}\right)$. HRMS (APCI +): Calculated for $\mathrm{C}_{8} \mathrm{H}_{10} \mathrm{NS}\left(\mathrm{M}-\mathrm{N}_{2}+\mathrm{H}^{+}\right)$: 152.0528 ; Found: 152.0525 .

(azidomethyl)(phenyl)sulfane (3dd)<smiles>N#CSc1ccccc1</smiles>
A vial containing $\mathrm{Cu}(\text { dap })_{2} \mathrm{Cl}(0.005$ equiv, $0.003 \mathrm{mmol}, 2.20 \mathrm{mg}), \quad 1$-azido- $1 \lambda^{3}-$ benzo[ $d][1,2]$ iodaoxol-3(1H)-one (1.00 equiv, $0.50 \mathrm{mmol}, 145 \mathrm{mg}$ ) and equipped with a stirrer bar, sealed with a septum, was purged under a flux of nitrogen. Then degassed mixture of acetonitrile $(2.5 \mathrm{~mL})$ and thianisole ( 40.0 equiv, $20.0 \mathrm{mmol}, 2.35 \mathrm{~mL}$ ) was added and the tube was sealed and put one centimeter away from a $30 \mathrm{~W}$ fluorescent bulb and stirred for 16 hours. The crude reaction mixture was loaded directly on the chromatography column and purified with mixtures of hexane:DCM affording $\mathbf{3} \boldsymbol{d} \boldsymbol{d}$ as a colourless oil $(32.0 \mathrm{mg}, 67 \%)$. Data are in accordance with previous literature reports. ${ }^{22}{ }^{1} \mathrm{H}$ NMR (400 $\mathrm{MHz}$, Chloroform- $d$ ) $\delta$ 7.51-7.48 (m, 2H), 7.37-7.28 (m, 3H), $4.54(\mathrm{~s}, 1 \mathrm{H}) .{ }^{13} \mathrm{C}$ NMR $(101 \mathrm{MHz}$, Chloroform- $d$ ) $\delta 133.6,131.3,129.4,127.9,56.1$. IR (neat film): 2920, 2097, 1222, 1196, 864, 739, 689. MS $($ APCI +$)$ : $138\left(\mathrm{M}-\mathrm{N}_{2}+\mathrm{H}^{+}\right)$. HRMS $\left(\right.$APCI + ): Calculated for $\mathrm{C}_{7} \mathrm{H}_{8} \mathrm{NS}\left(\mathrm{M}-\mathrm{N}_{2}+\mathrm{H}^{+}\right)$: 138.0372; Found: 138.0374 .

(azidomethyl)(4-bromophenyl)sulfane (3ee)

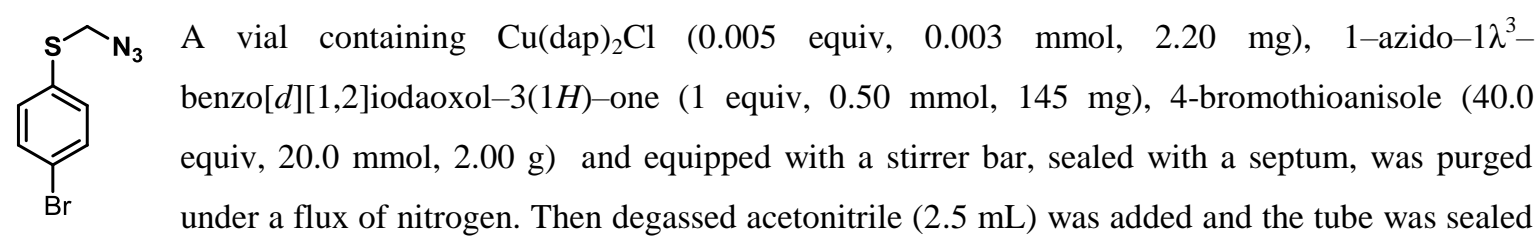
and put 1.0 centimeter away from a $30 \mathrm{~W}$ fluorescent bulb and stirred for 16 hours. The crude reaction mixture was loaded directly on the chromatography column and purified with mixtures of hexane:EtOAc 
affording $3 \boldsymbol{e e}$ as a colourless oil (32.0 mg, 53\%). ${ }^{1} \mathrm{H}$ NMR (400 MHz, Chloroform- $d$ ) $\delta$ 7.48-7.46 (m, 2H), 7.37-7.35 (m, 2H), 4.52 (s, 2H). ${ }^{13} \mathrm{C}$ NMR (101 MHz, Chloroform- $d$ ) $\delta 132.8,132.5,122.2,100.1,56.0$. IR (neat film): 2918, 2360, 2101, 1473, 1224, 1090, 1068, 1008, 865, 809. MS (APCI +): $218\left({ }^{81} \mathrm{BrM}-\mathrm{N}_{2}+\right.$ $\left.\mathrm{H}^{+}\right), 216\left({ }^{79} \mathrm{BrM}-\mathrm{N}_{2}+\mathrm{H}^{+}\right)$. HRMS (APCI + ): Calculated for $\mathrm{C}_{7} \mathrm{H}_{7} \mathrm{NBrS}\left(\mathrm{M}-\mathrm{N}_{2}+\mathrm{H}^{+}\right)$: 215.9477; Found: 215.9481 .

2-(azidomethyl)-1,3-difluorobenzene (3ff)

$\mathrm{A}$ vial containing $\mathrm{Cu}(\mathrm{dap})_{2} \mathrm{Cl}(0.005$ equiv, $0.003 \mathrm{mmol}, 2.20 \mathrm{mg}), 1$-azido- $1 \lambda^{3}-$
benzo $[d][1,2]$ iodaoxol-3(1H)-one $(1.00$ equiv, $0.50 \mathrm{mmol}, 145 \mathrm{mg})$ and equipped with a
stirrer bar, sealed with a septum, was purged under a flux of nitrogen. Then degassed mixture of acetonitrile $(2.5 \mathrm{~mL})$ and 1,3-difluoro-2-methylbenzene (40.0 equiv, $20.0 \mathrm{mmol}, 2.25 \mathrm{~mL}$ ) was added and the tube was sealed and put one centimeter away from a $30 \mathrm{~W}$ fluorescent bulb and stirred for 16 hours. The crude reaction mixture was loaded directly on the chromatography column and purified with mixtures of hexane:DCM affording $3 f f$ as a colourless oil (32.0 mg, 38\%). Data are in accordance with previous literature reports. ${ }^{23}{ }^{1} \mathrm{H}$ NMR (500 MHz, Chloroform- $d$ ) $\delta 7.33(\mathrm{~m}, 1 \mathrm{H}), 6.97-6.94(\mathrm{~m}, 2 \mathrm{H}), 4.43(\mathrm{~s}, 2 \mathrm{H}) .{ }^{13} \mathrm{C} \mathrm{NMR}$ $(126 \mathrm{MHz}$, Chloroform- $d) \delta 161.7(\mathrm{dd}, J=250.7,7.4 \mathrm{~Hz}), 130.8(\mathrm{t}, J=10.0 \mathrm{~Hz}), 111.7(\mathrm{dd}, J=19.9 \mathrm{~Hz}, 5.5$ $\mathrm{Hz}), 111.7(\mathrm{t}, J=19.5 \mathrm{~Hz}), 42.0(\mathrm{t}, J=3.1 \mathrm{~Hz}) .{ }^{19} \mathrm{~F}$ NMR $(471 \mathrm{MHz}$, Chloroform- $d$ ) -114.7 (t, $J=7.0 \mathrm{~Hz})$. IR (neat film): 2929, 2097, 1627, 1471, 1237, 1040, 783.

Rufinamide (4)

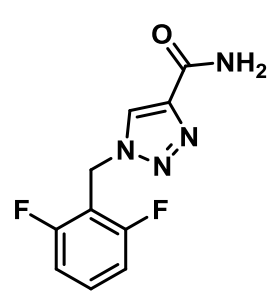

2-(azidomethyl)-1,3-difluorobenzene (1.00 equiv, $0.17 \mathrm{mmol}, 29 \mathrm{mg}$ ) was put in solution in ethanol. $\mathrm{CuSO}_{4}(\mathrm{H} 2 \mathrm{O})_{5}(0.01$ equiv, $0.002 \mathrm{mmol}, 0.30 \mathrm{mg})$, ascorbic acid ( 0.10 equiv, $0.02 \mathrm{mmol}, 3.24 \mathrm{mg}$ ) were added to the solution in one portion and methyl propiolate (1.50 equiv, $0.27 \mathrm{~m} \mathrm{~mol}, 24.0 \mu \mathrm{L}$ ) were added dropwise. The reaction was stirred at room temperature for 36 hours. The reaction was vacced down and taken up with a solution of ammonia in methanol (14.0 equiv, $2.48 \mathrm{mmol}, 105 \mu \mathrm{L}, 7 \mathrm{M})$ and left stirred for 72 hours. The reaction mixture was vacced off and the title compound was obtained as a white solid $(41.0 \mathrm{mg}, 100 \%, 38 \%$ across the three steps). Data are in accordance with previous literature reports. ${ }^{24}{ }^{1} \mathrm{H}$ NMR $\left(400 \mathrm{MHz}, \mathrm{DMSO}-d_{6}\right) \delta 8.56(\mathrm{~s}, 1 \mathrm{H})$, 7.89 (brs, 1H), 7.56-7.45 (m, 2H), 7.19 (t, $J=8.1 \mathrm{~Hz}, 2 \mathrm{H}), 5.72(\mathrm{~s}, 2 \mathrm{H}) .{ }^{13} \mathrm{C}$ NMR (101 MHz, DMSO- $\left.d_{6}\right) \delta$ $161.4,160.9$ (dd, $J=249.0,7.1 \mathrm{~Hz}), 142.9,131.9(\mathrm{t}, J=10.7 \mathrm{~Hz}), 126.9,112.1(\mathrm{dd}, J=18.7,5.5 \mathrm{~Hz}), 111.1$ (t. $J$ $=19.1 \mathrm{~Hz}), 41.2(\mathrm{t}, J=3.7 \mathrm{~Hz}) .{ }^{19} \mathrm{~F}$ NMR $\left(376 \mathrm{MHz}, \mathrm{DMSO}-d_{6}\right)-114.6(\mathrm{t}, J=7.2 \mathrm{~Hz})$. MS (APCI +$): 239(\mathrm{M}$ $+\mathrm{H})$. HRMS (APCI +): Calculated for $\mathrm{C}_{10} \mathrm{H}_{8} \mathrm{~F}_{2} \mathrm{~N}_{4} \mathrm{O}\left(\mathrm{M}+\mathrm{H}^{+}\right)$: 239.0739; Found: 239.0734. 
${ }^{1} \mathrm{H}$ NMR and ${ }^{13} \mathrm{C}$ NMR for azidated substrates $3 a$ to 4

Ba

lH NMR, $400 \mathrm{MHz}, \mathrm{CDCl} 3$

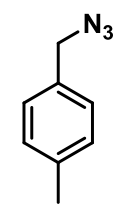

$\underbrace{3 \pi \sqrt{3}}$

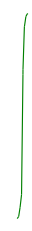

$\stackrel{\text { ริ }}{\text { । }}$

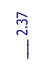

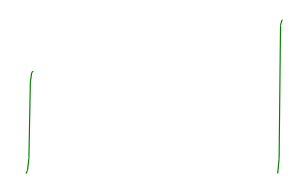

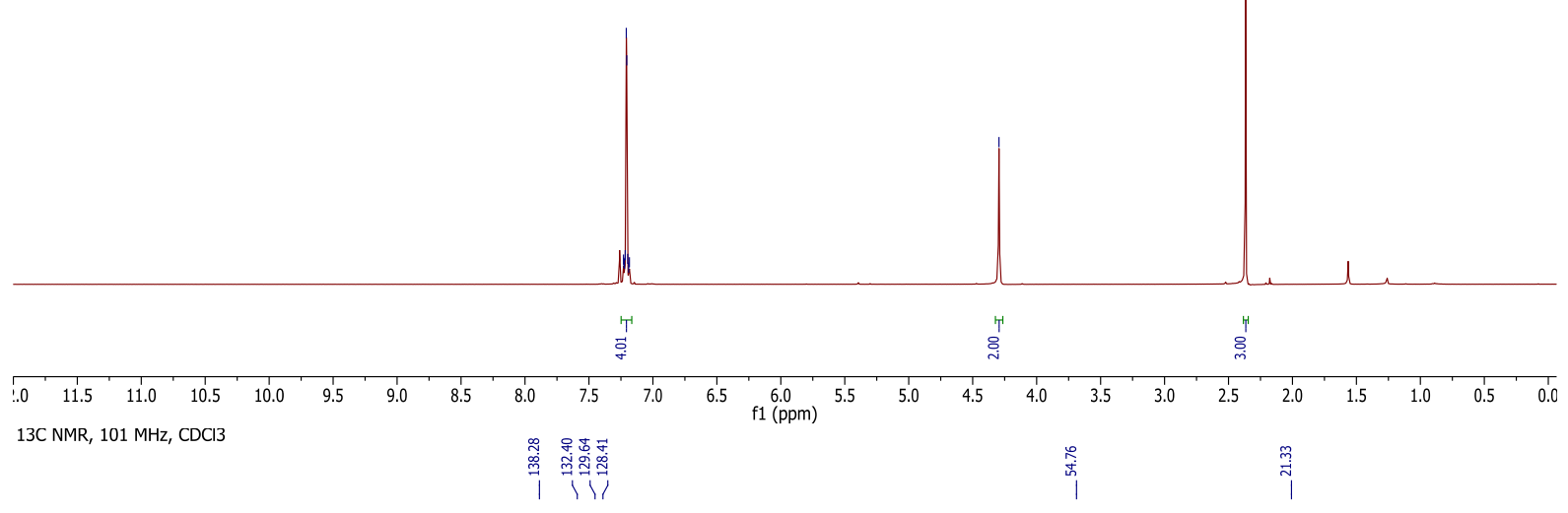
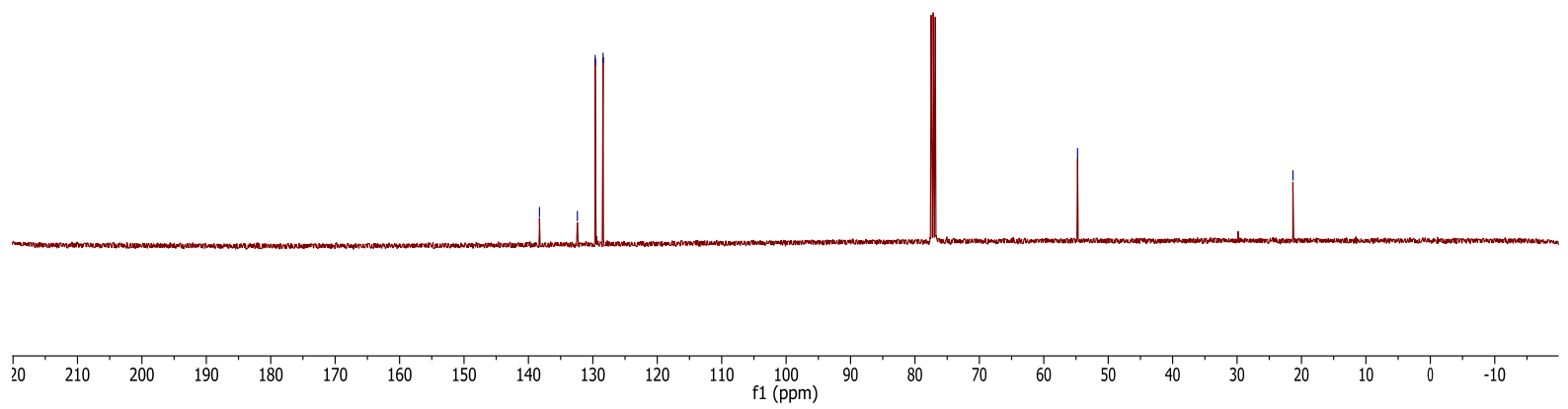

S16 
3b

1H NMR, $400 \mathrm{MHz}, \mathrm{CDCl} 3$

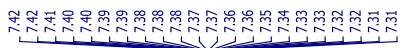

学
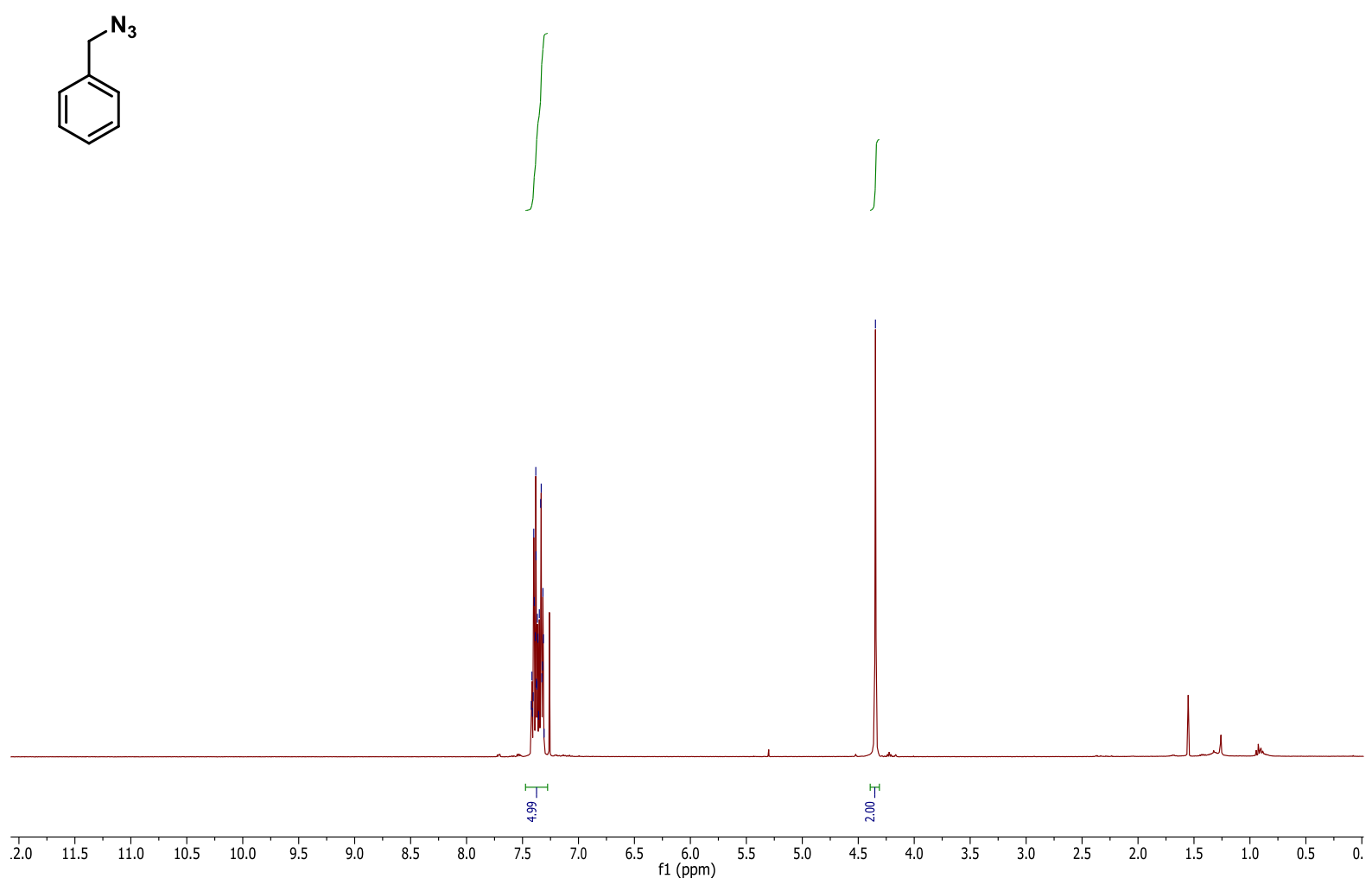

13C NMR, $101 \mathrm{MHz}, \mathrm{CDCl} 3$

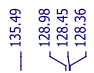

量
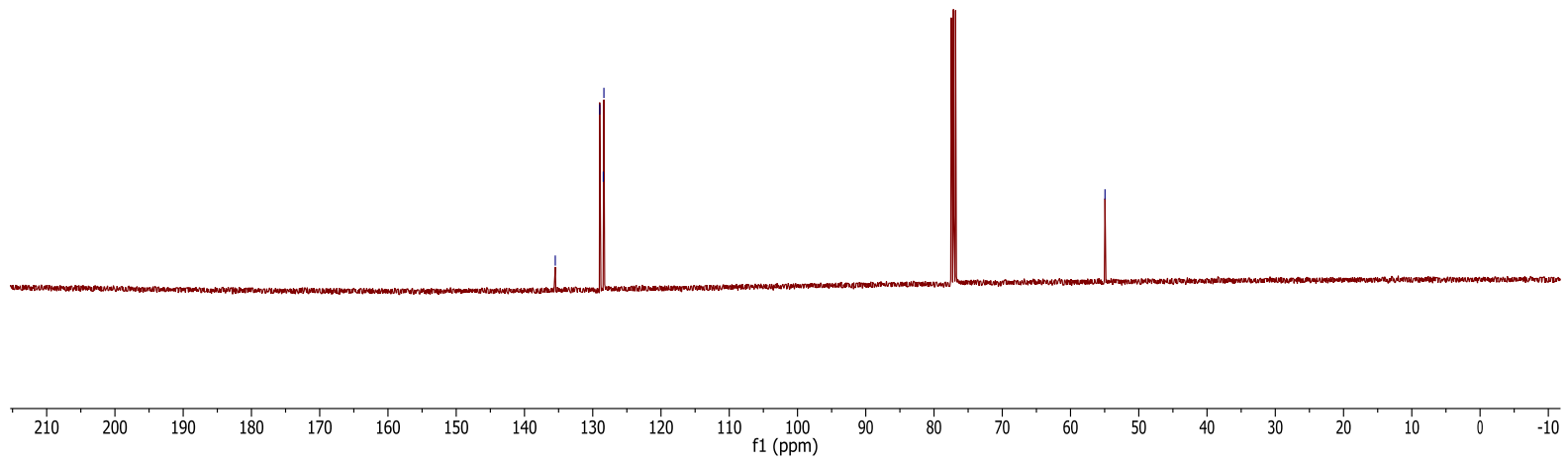

S17 
$3 c$

$1 \mathrm{H}$ NMR, $400 \mathrm{MHz}, \mathrm{CDCl} 3$

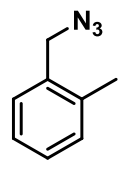

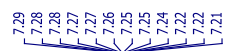

孚

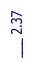
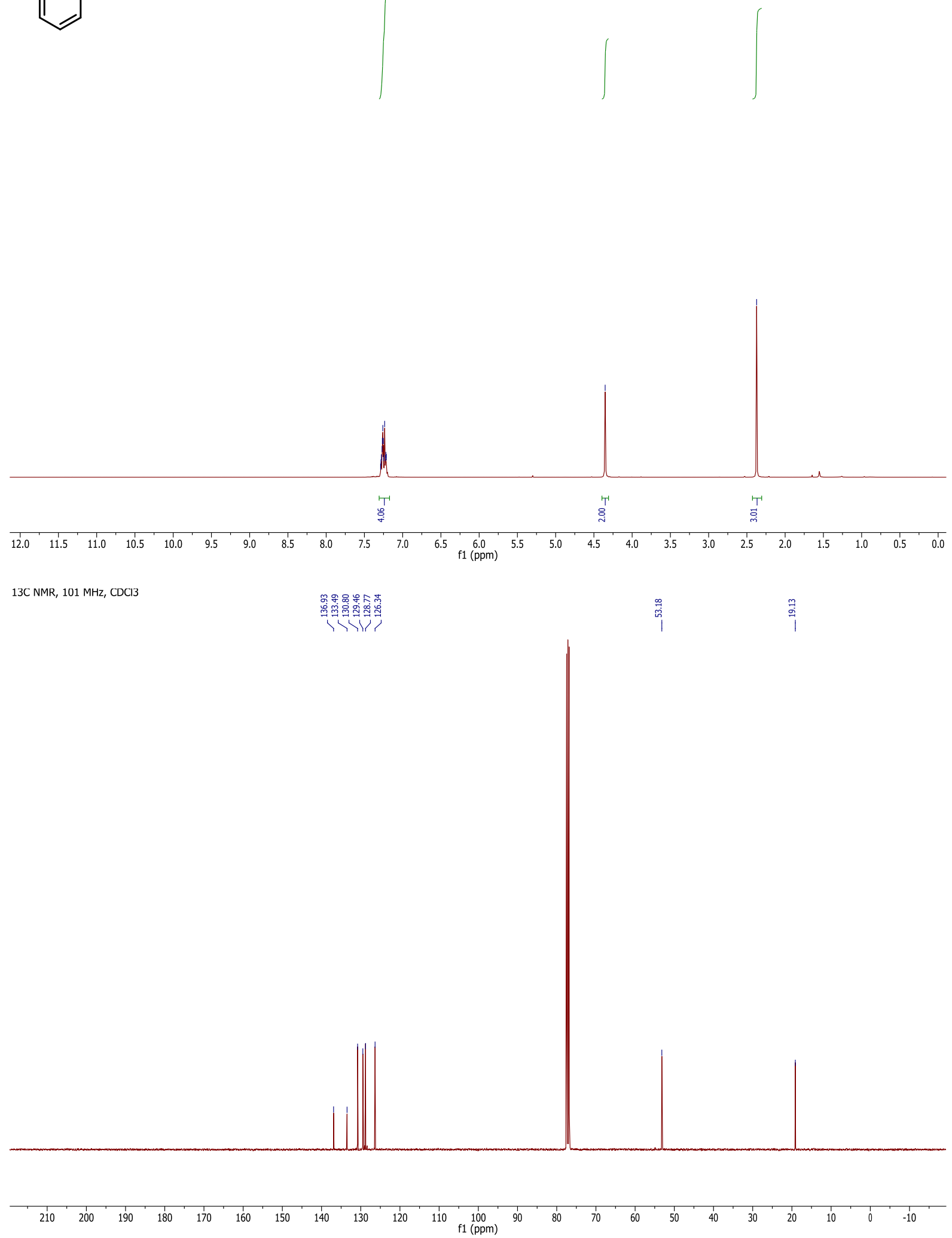

S18 
3d
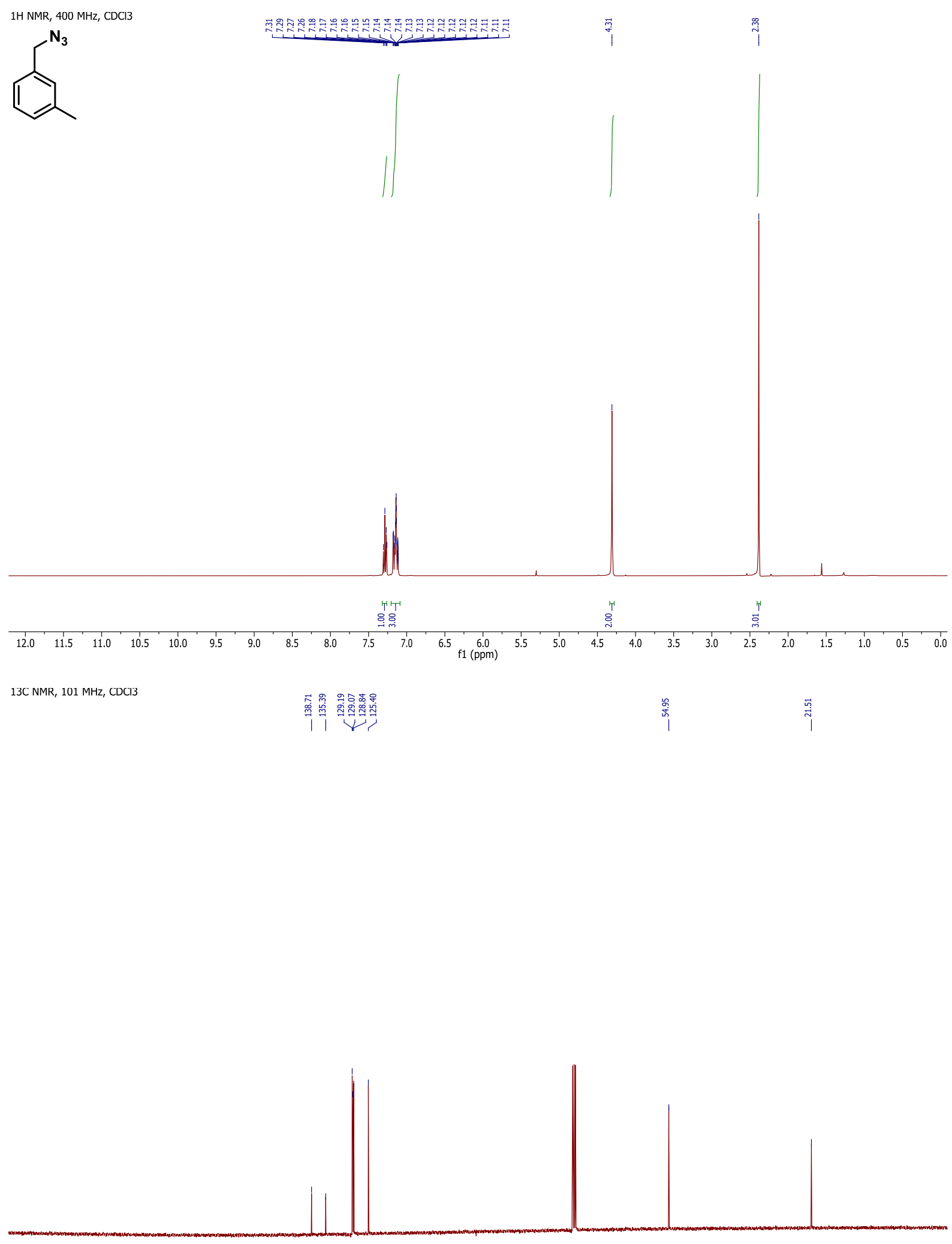

S19 
3e

1H NMR, $400 \mathrm{MHz}$, CDCl3<smiles>[N]Cc1ccccc1Br</smiles>

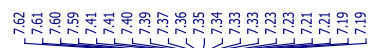

ชั
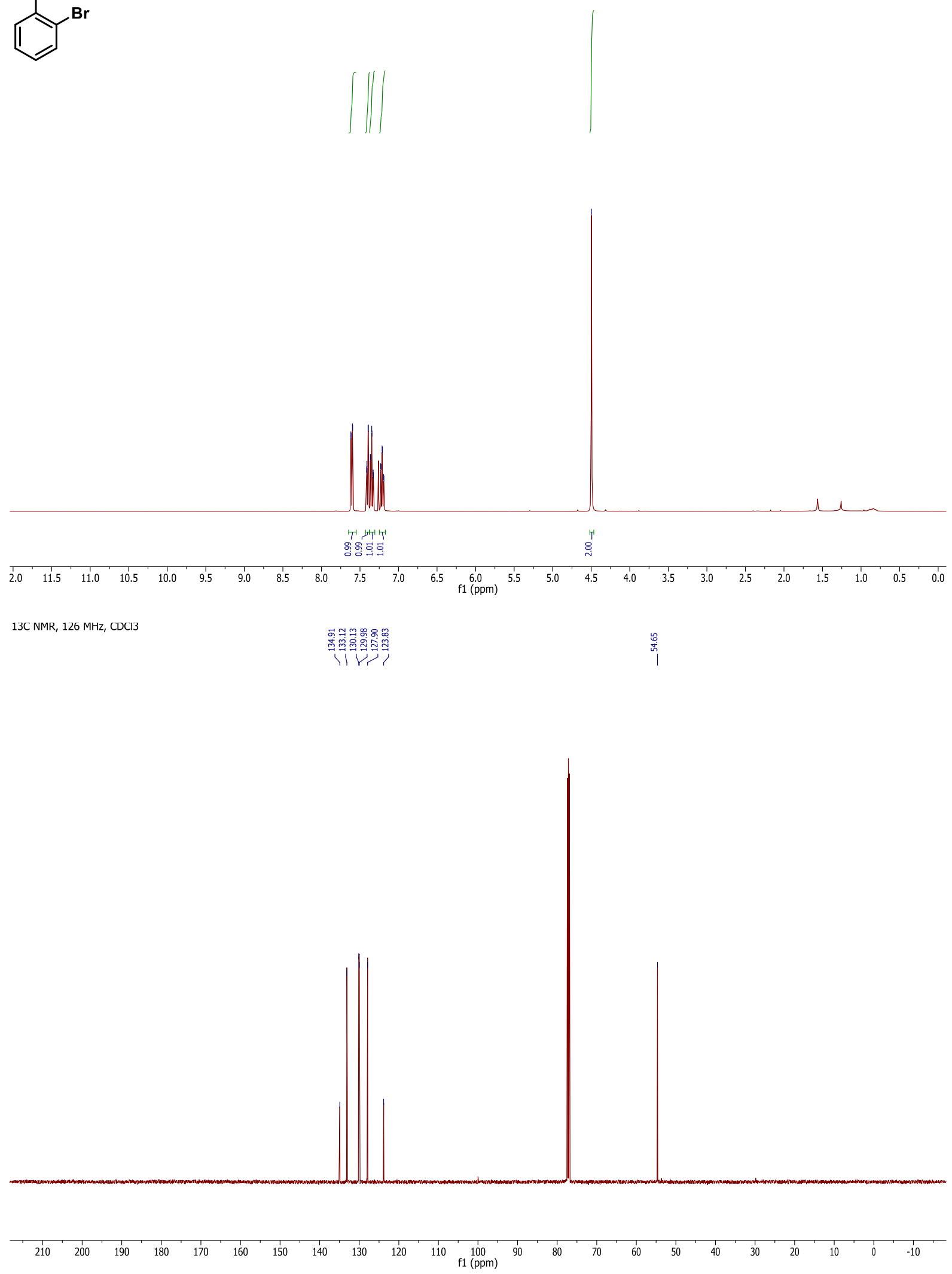

S20 
3f

1H NMR, 400 MHz, CDCl3
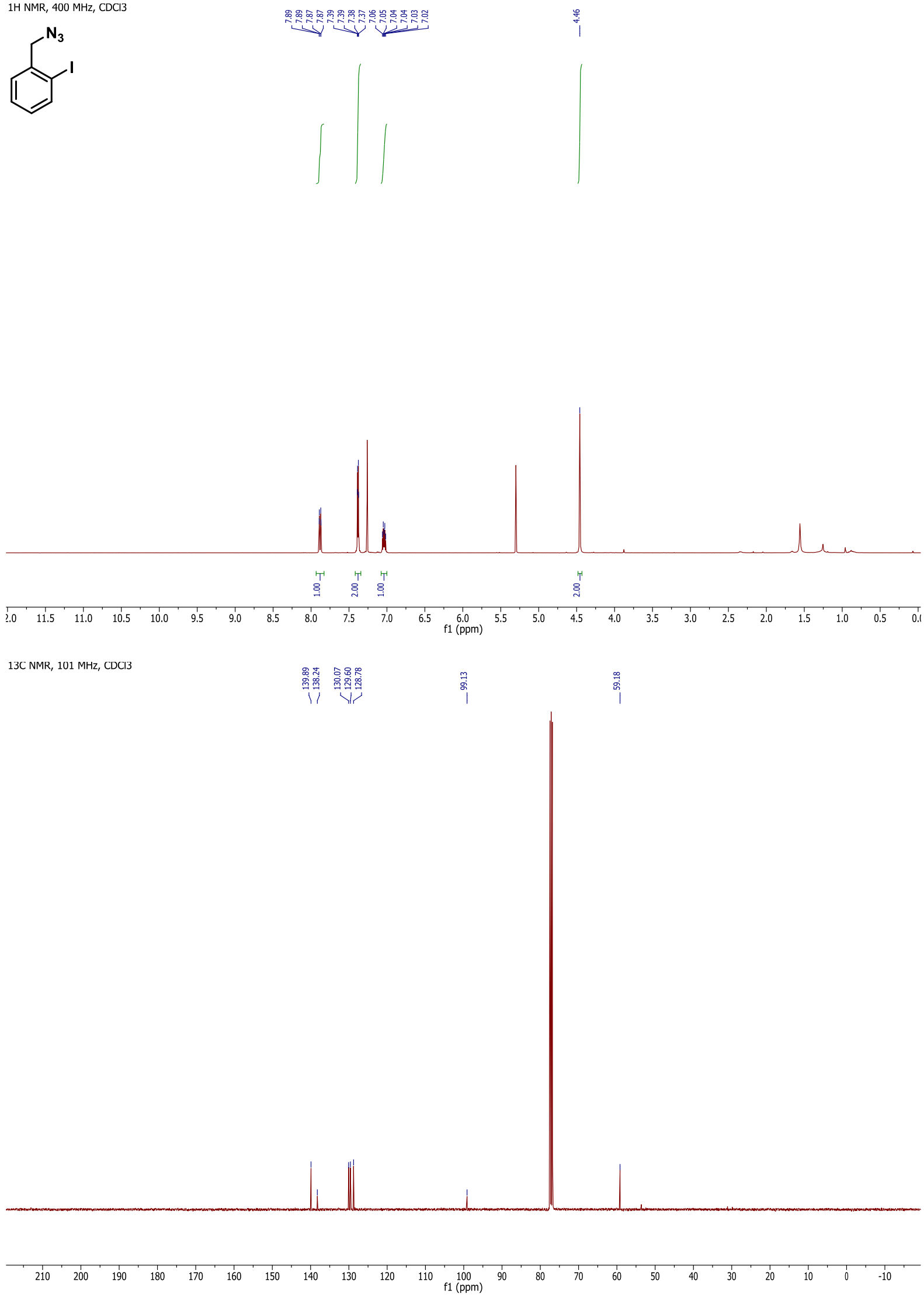

S21 
$3 g$

$1 \mathrm{H} \mathrm{NMR,} 400 \mathrm{MHz}$, CDC

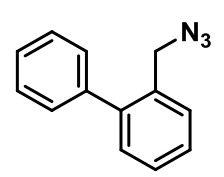

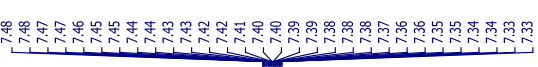

i
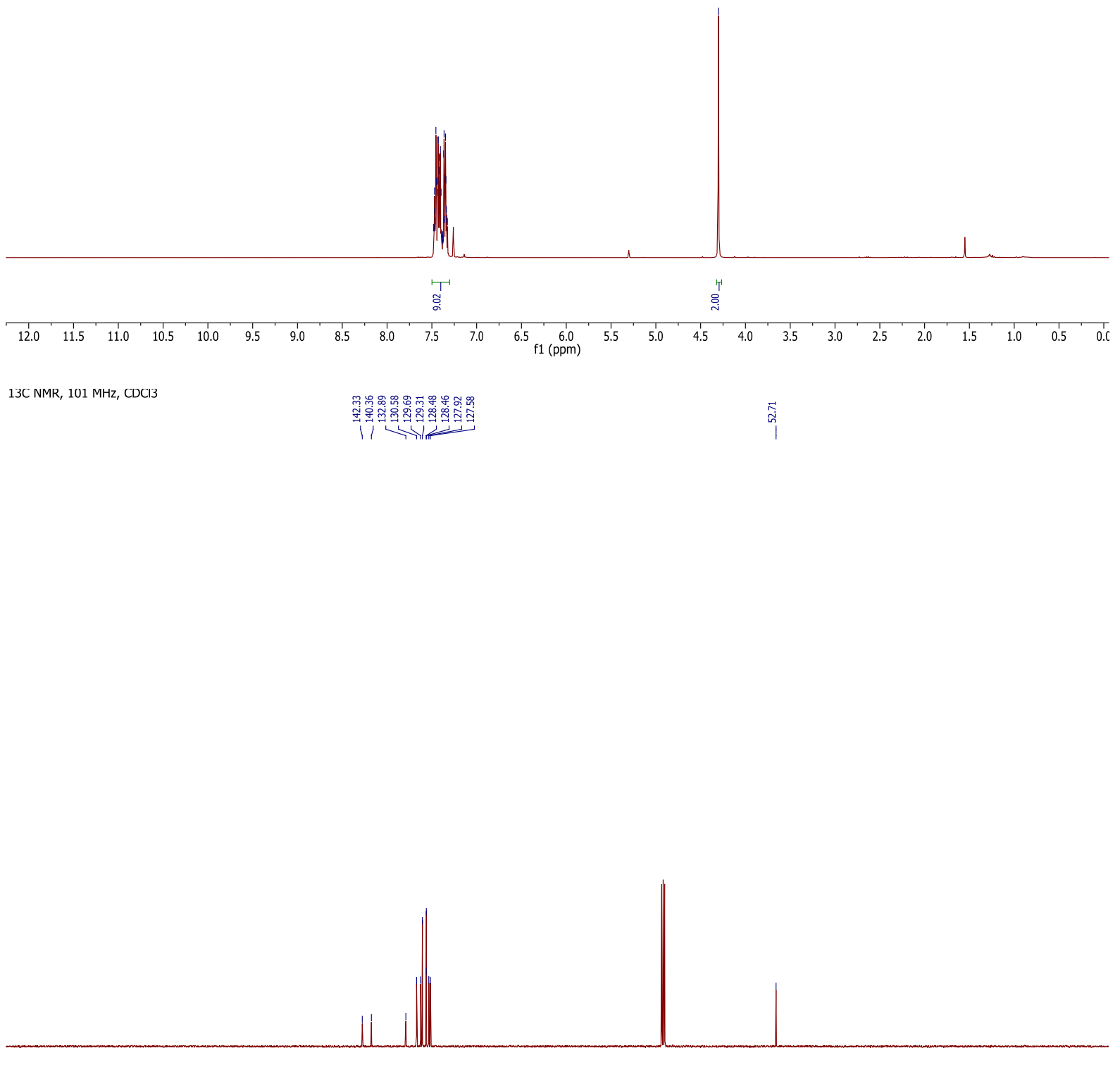

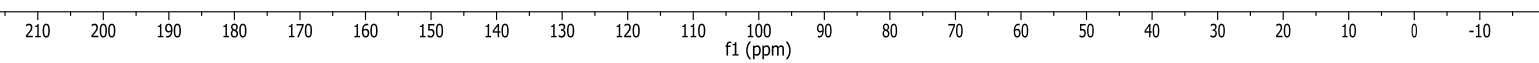

S22 
$3 h$

$1 \mathrm{H} \mathrm{NMR,} 400 \mathrm{MHz}, \mathrm{CDCl} 3$

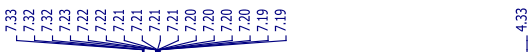<smiles>N#Cc1cccc(Cl)c1</smiles>
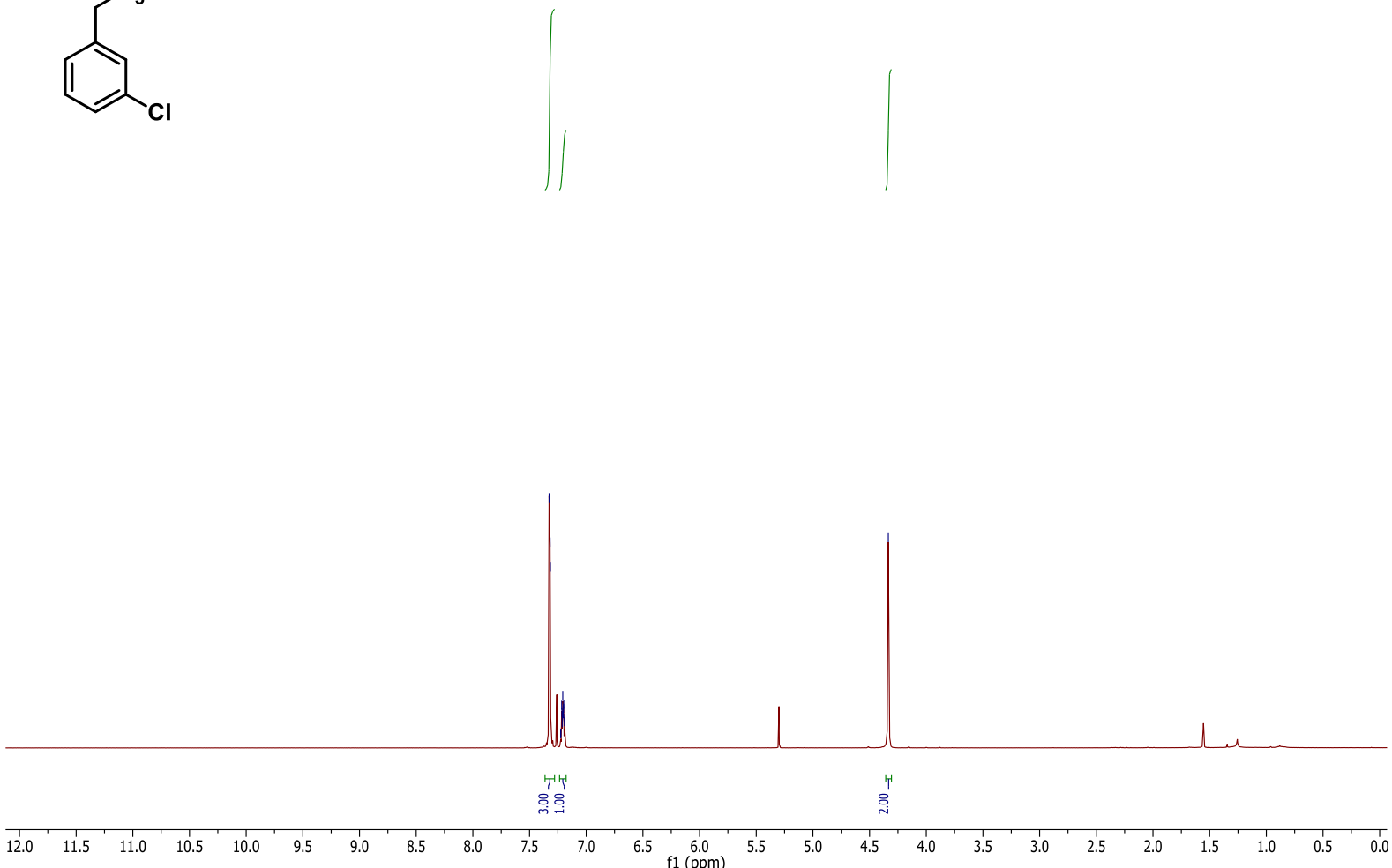

$13 \mathrm{C}$ NMR, $101 \mathrm{MHZ}, \mathrm{CDCl3}$

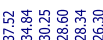

의궈

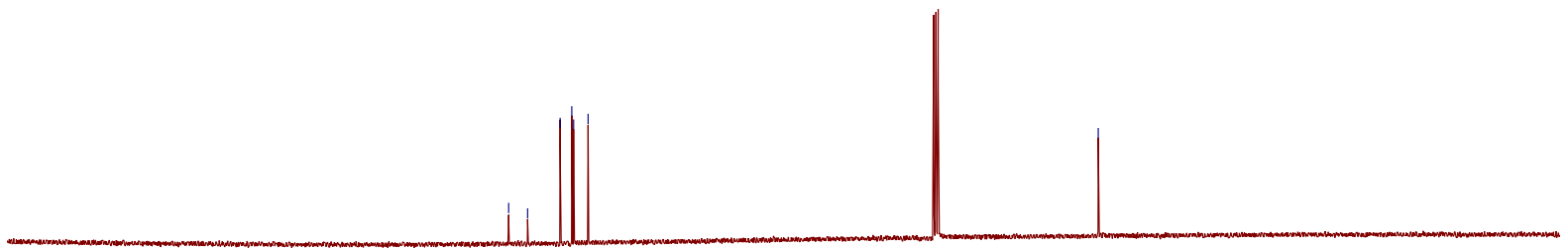

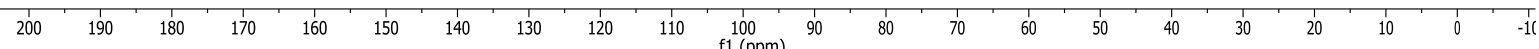


$3 i$

$1 \mathrm{H}$ NMR, $400 \mathrm{MHz}$, CDCl3
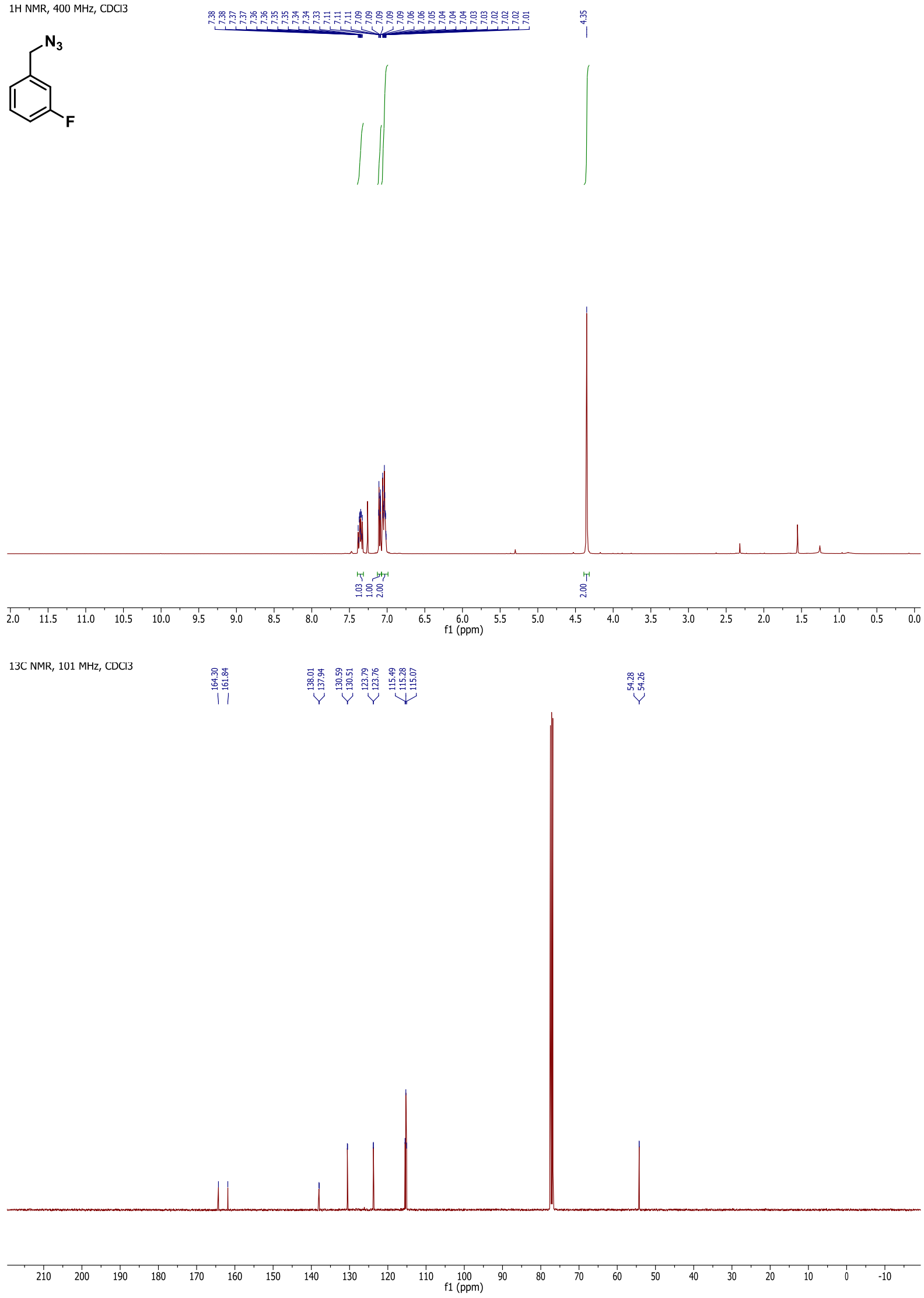

S24 
$19 F \mathrm{NMR}, 376 \mathrm{MHz}, \mathrm{CDCl} 3$

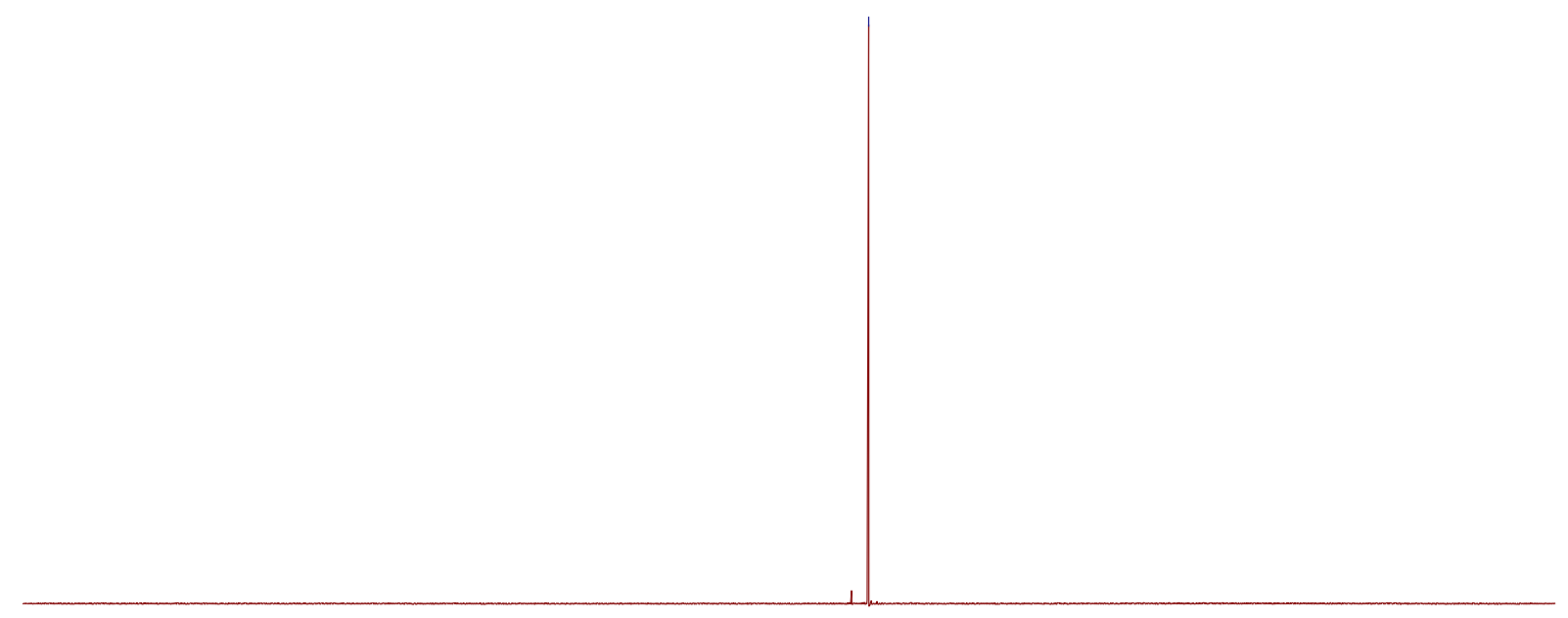

\begin{tabular}{rllllllllllllllllllllll}
\hline 10 & 0 & -10 & -20 & -30 & -40 & -50 & -60 & -70 & -80 & -90 & -100 & -110 & -120 & -130 & -140 & -150 & -160 & -170 & -180 & -190 & -200 & -210
\end{tabular}

3j

$1 \mathrm{H} \mathrm{NMR}, 400 \mathrm{MHz}, \mathrm{CDCl} 3$<smiles>N#Cc1ccc([N+](=O)[O-])cc1</smiles>

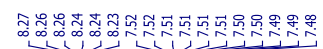

ป
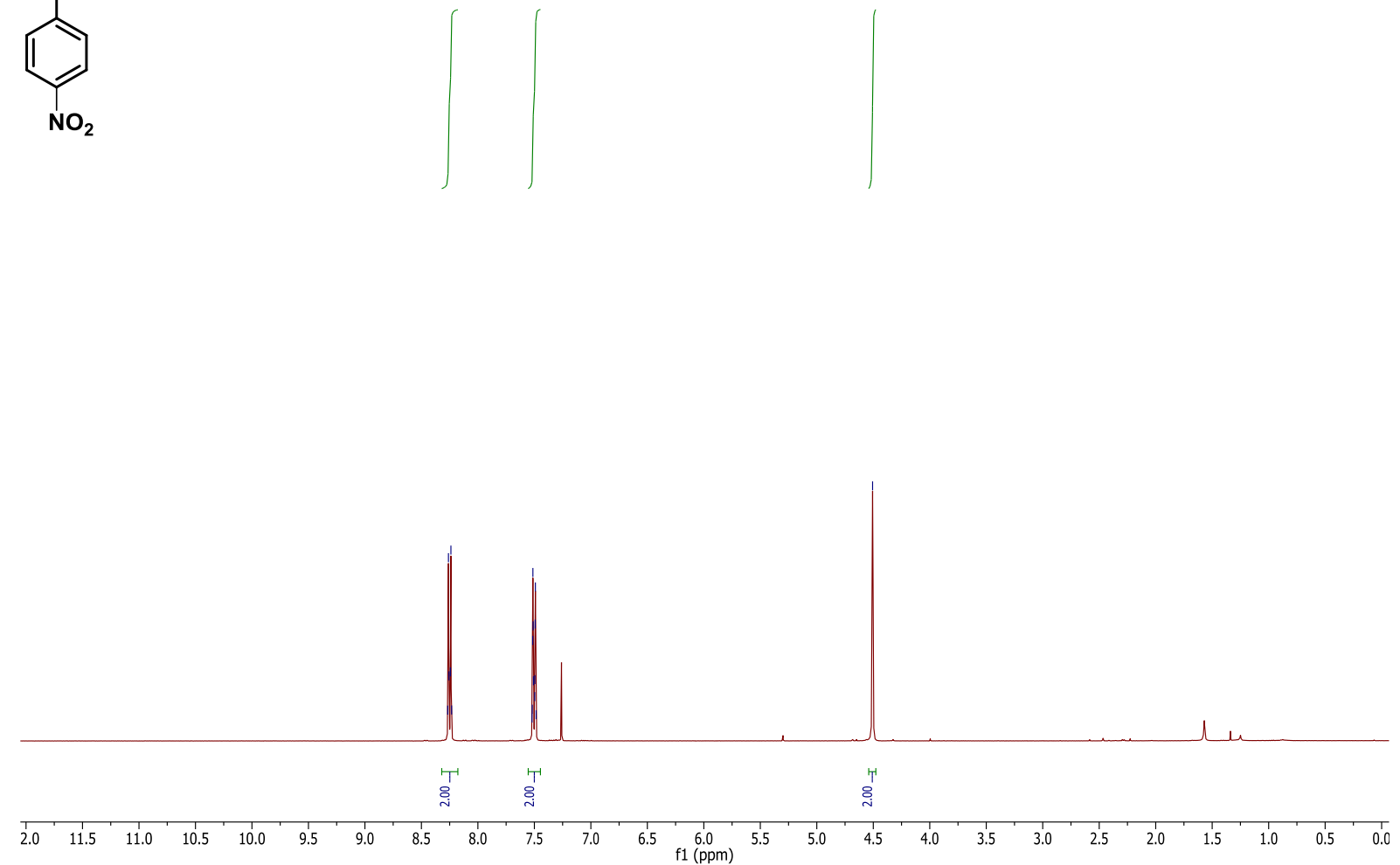
3j

13C NMR, 101 MHz, CDCl3

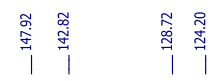

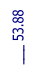
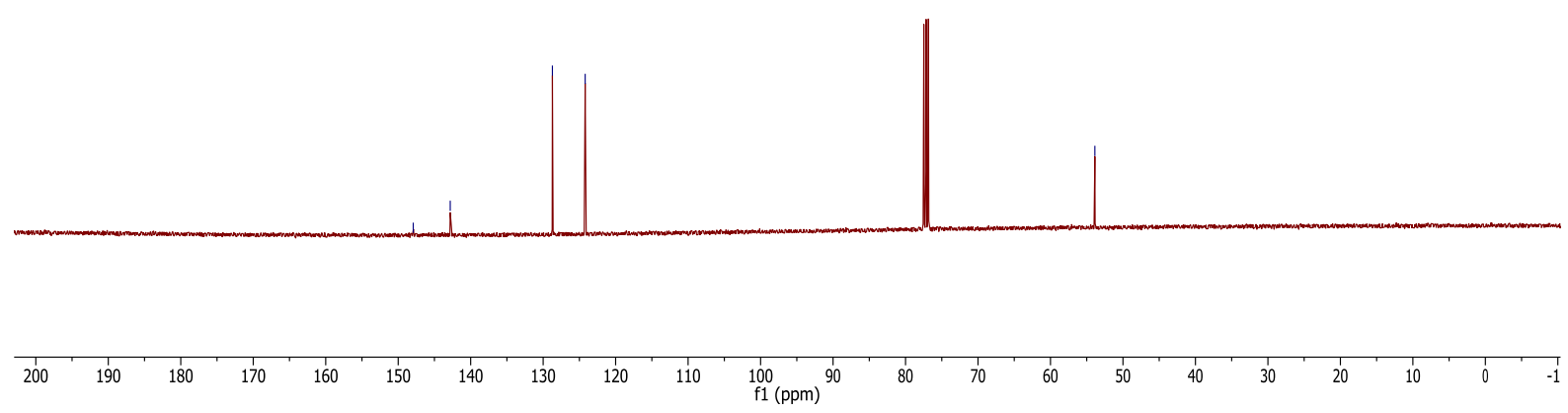

$3 \mathbf{k}$

1H NMR, 400 MHz, CDCl3

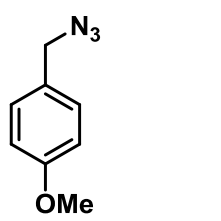

舟

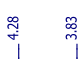

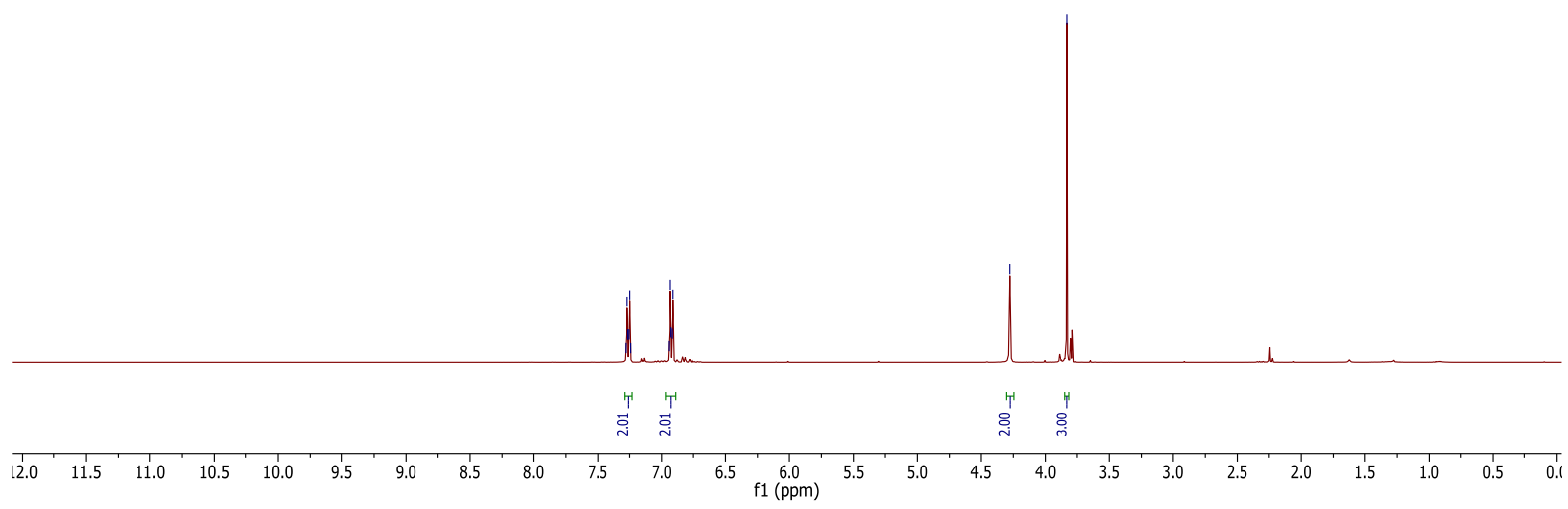

S26 
$3 \mathbf{k}$

13C NMR, 101 MHz, CDCl3

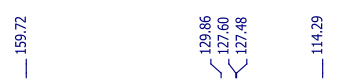

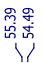

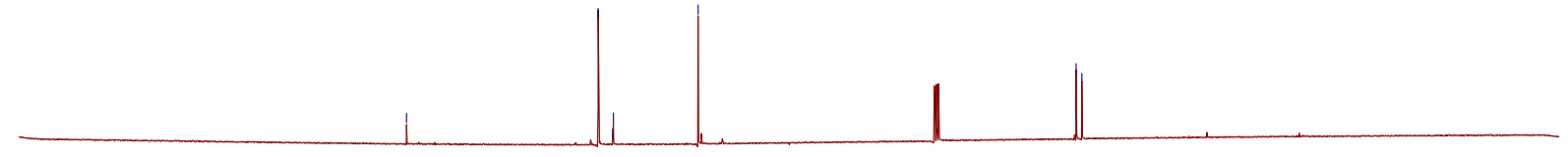

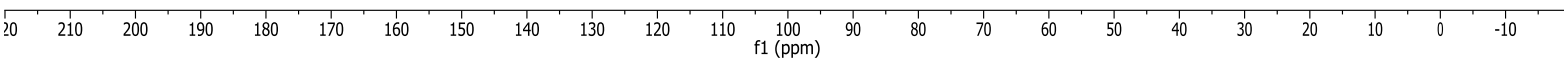

31

$1 \mathrm{H} \mathrm{NMR}, 500 \mathrm{MHz}, \mathrm{CDCl} 3$<smiles>N#Cc1ccc(CN)cc1</smiles>
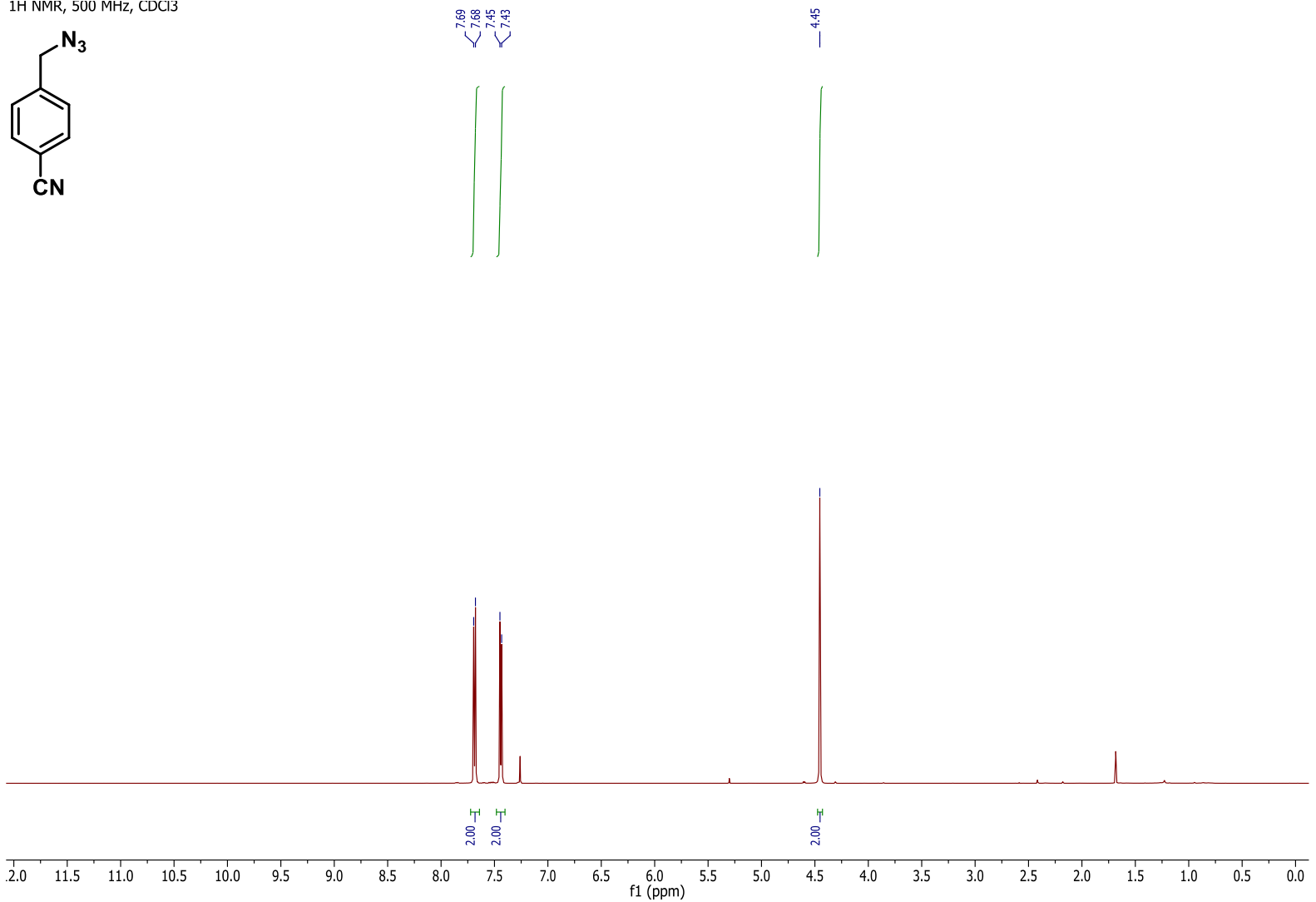
31

13C NMR, 126 MHz, CDCl3

।

离

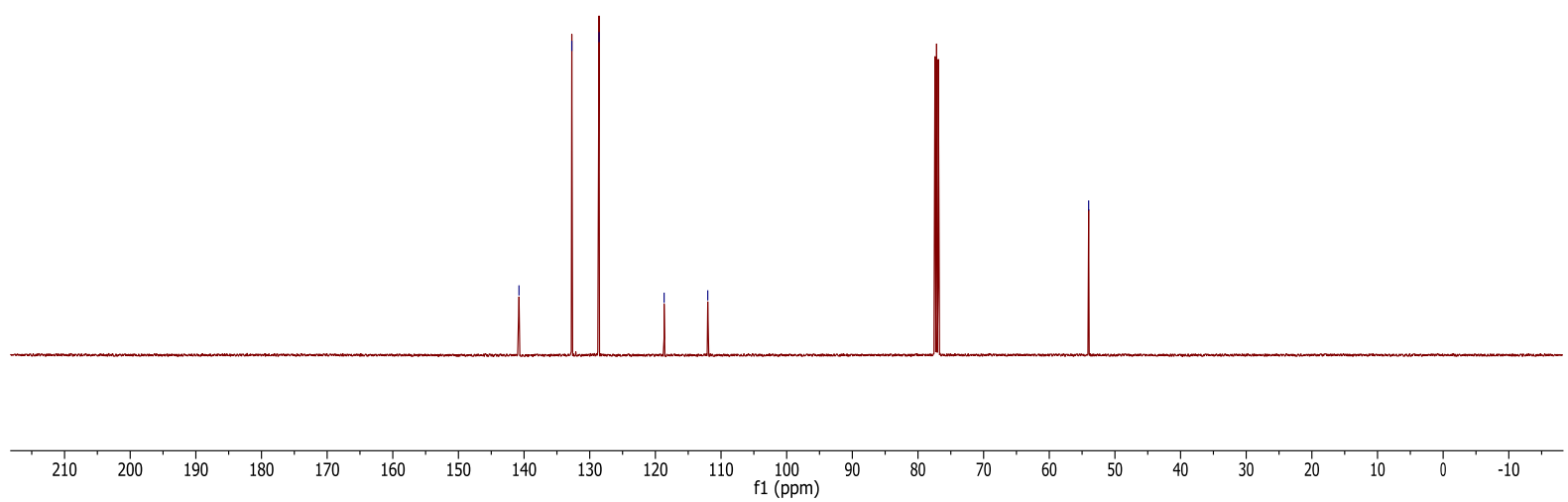

$3 m$

1H NMR, 400 MHz, CDCl3

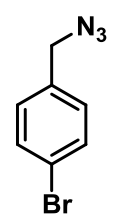

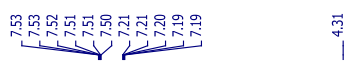

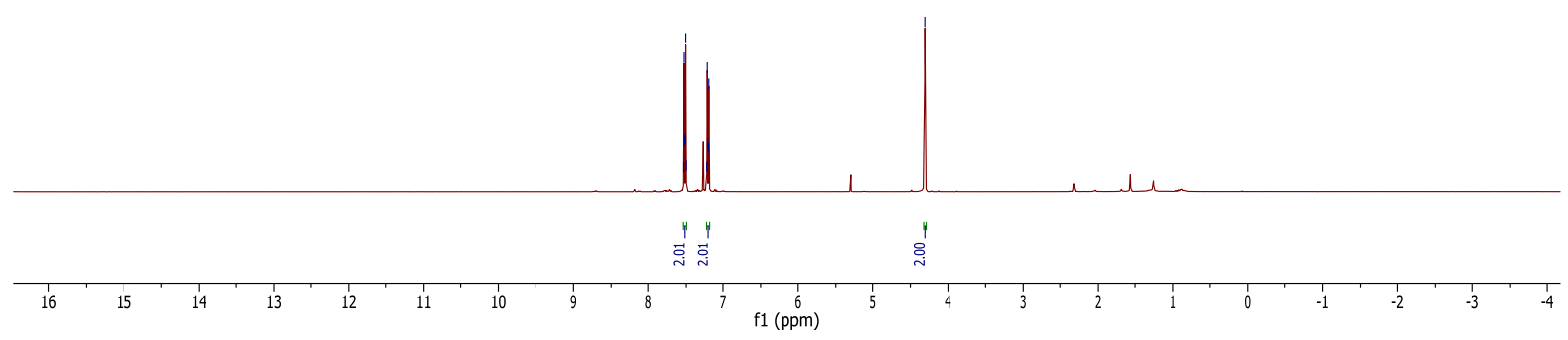

S28 
13C NMR, 101 MHz, CDCl3

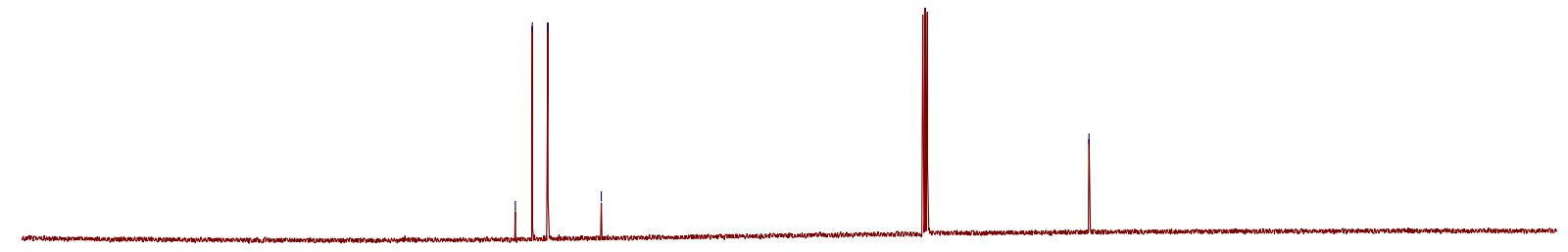

$$
200
$$

$\begin{array}{lllll}180 & 170 & 160 & 150 & 140\end{array}$

$120 \quad 110$

$100 \quad 90$

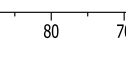

3n

$1 \mathrm{H} N \mathrm{NR}, 400 \mathrm{MHz}, \mathrm{CDCl} 3$<smiles>N#Cc1ccc(Cl)cc1</smiles>

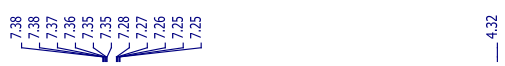
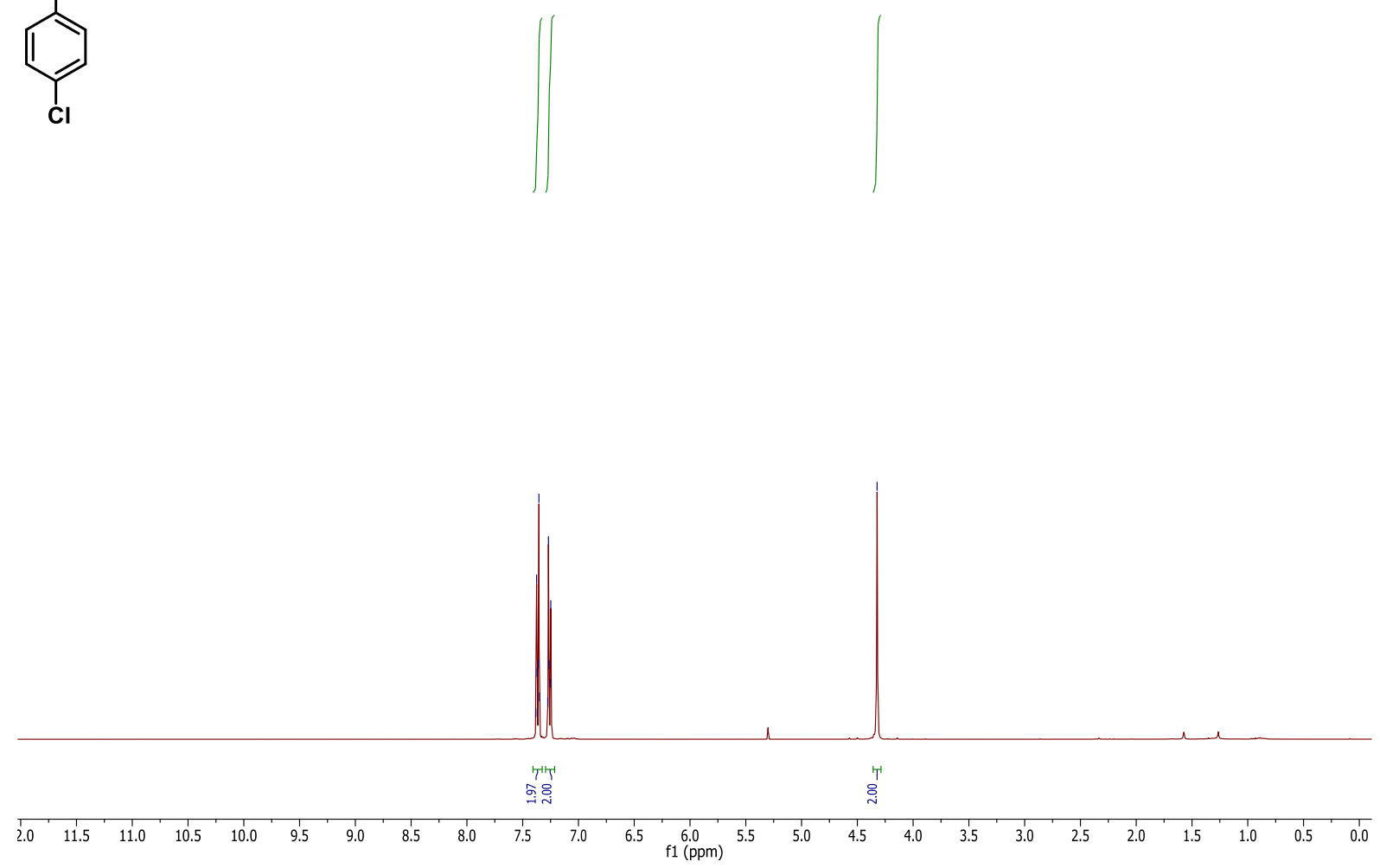
3n

13C NMR, $101 \mathrm{MHz}$, CDCl3

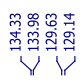

$\stackrel{n}{f^{\prime}}$

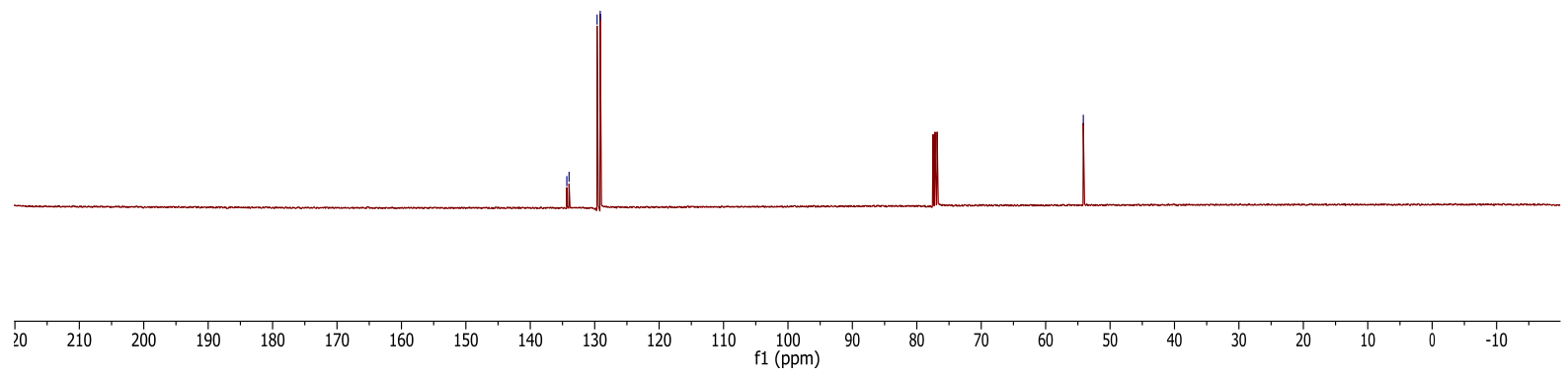

30

1H NMR, $400 \mathrm{MHz}, \mathrm{CDCl} 3$

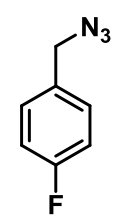

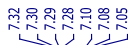

ซึ
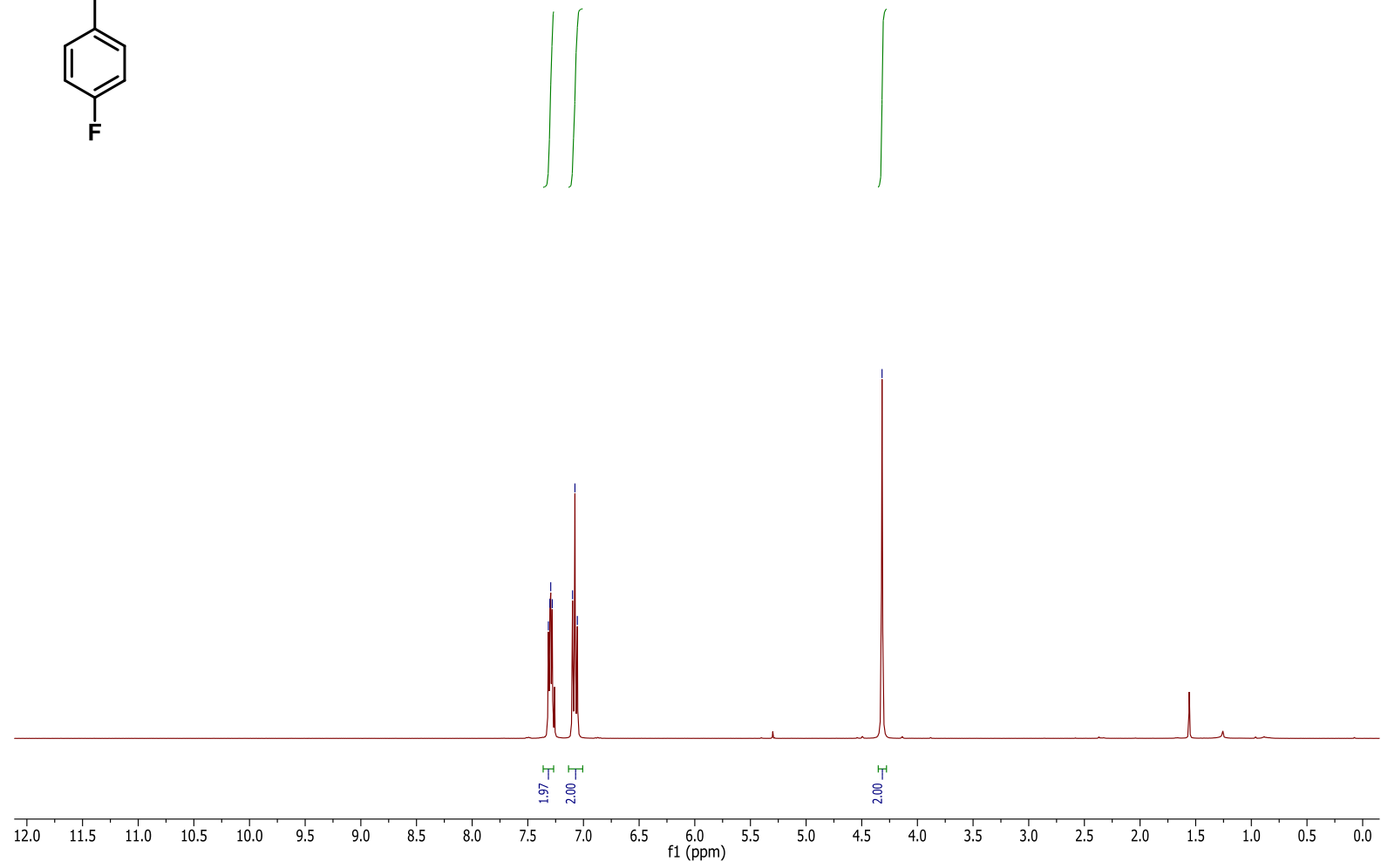

S30 
30

$13 \mathrm{C} \mathrm{NMR,} 101 \mathrm{MHz}, \mathrm{CDCl}$

西

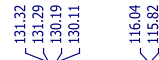

属

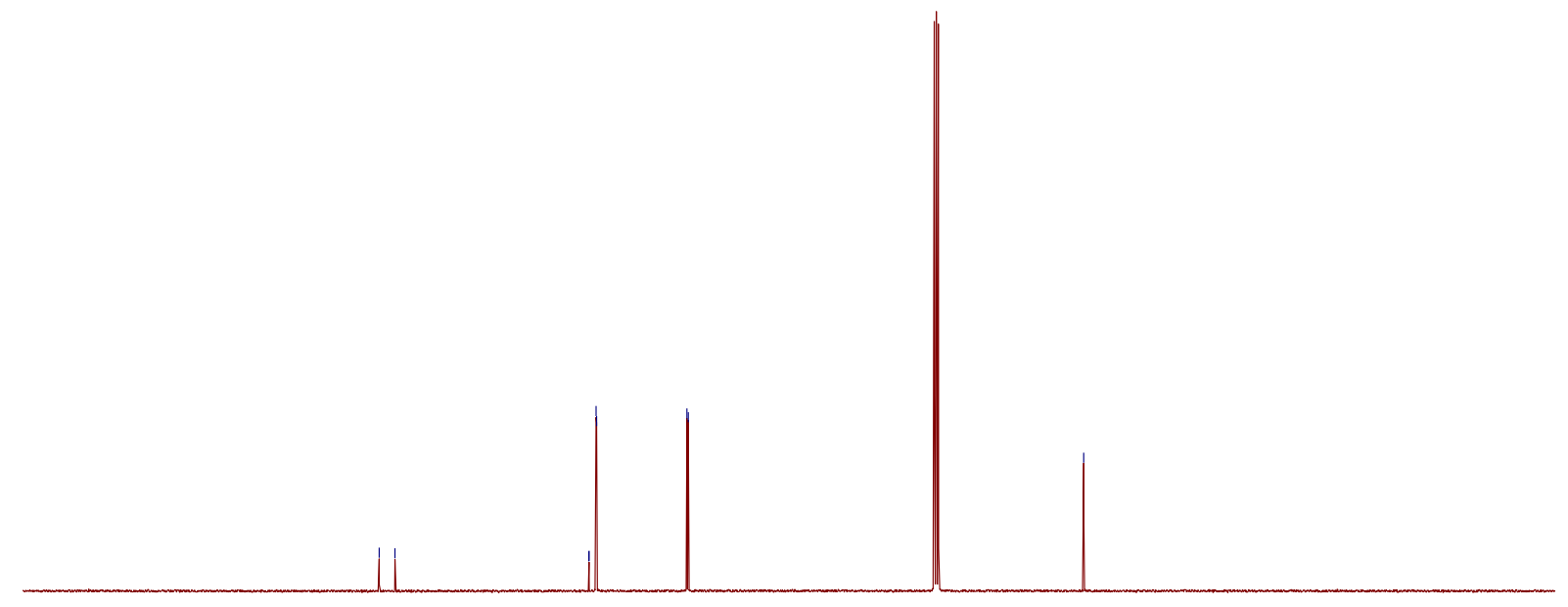

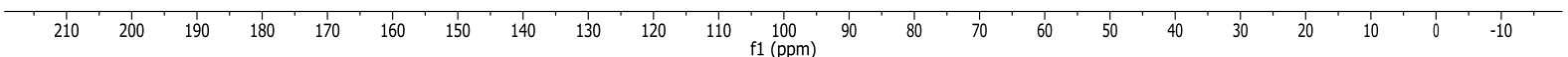

19F NMR, 376 MHz, CDCl3

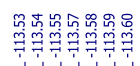

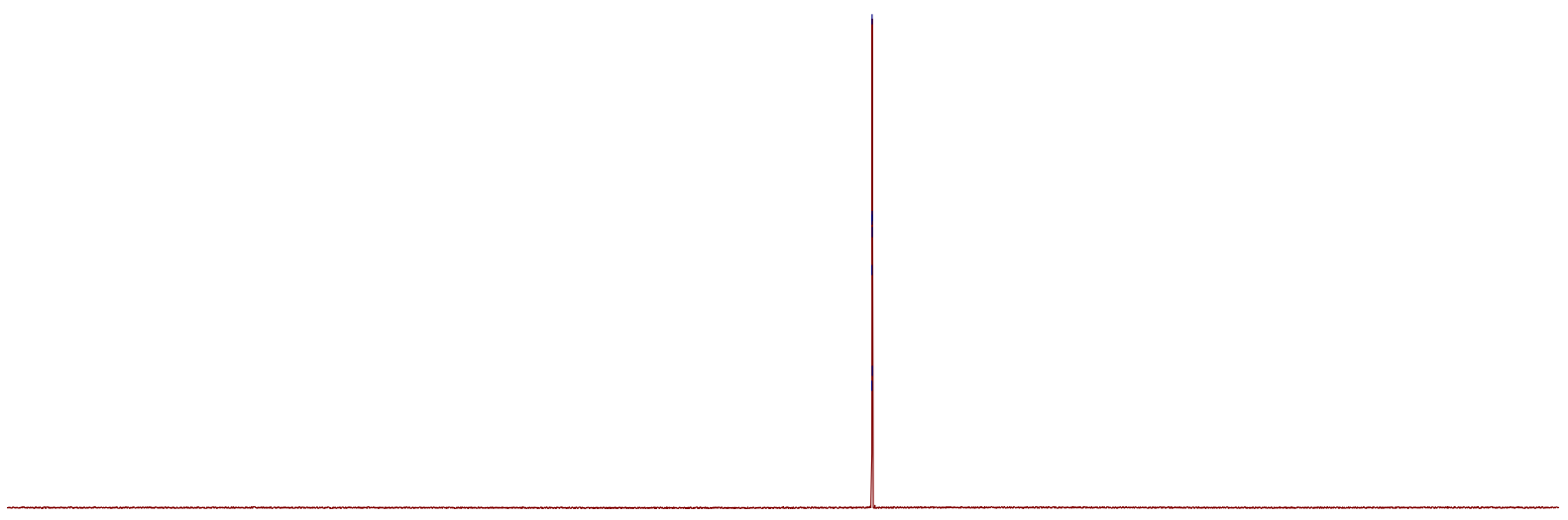

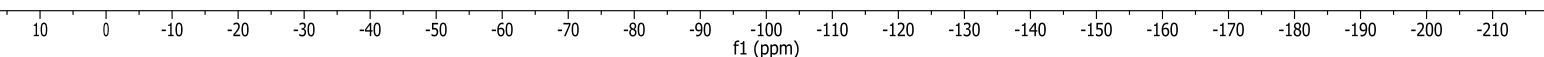


3p

1H NMR, 500 MHz, CDCl3
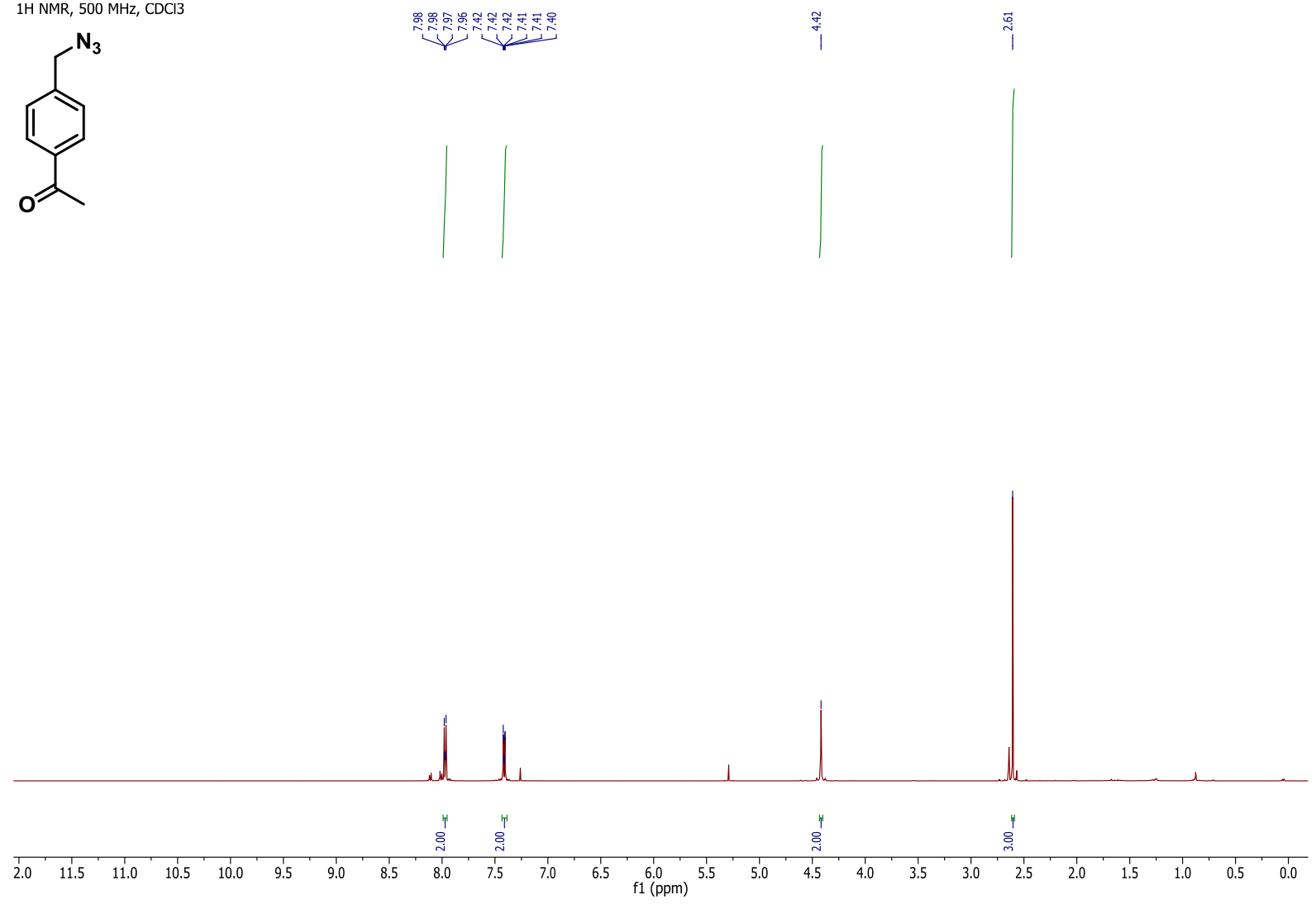

13C NMR, 126 MḦz, CDCl3

重量㖕

㬅管

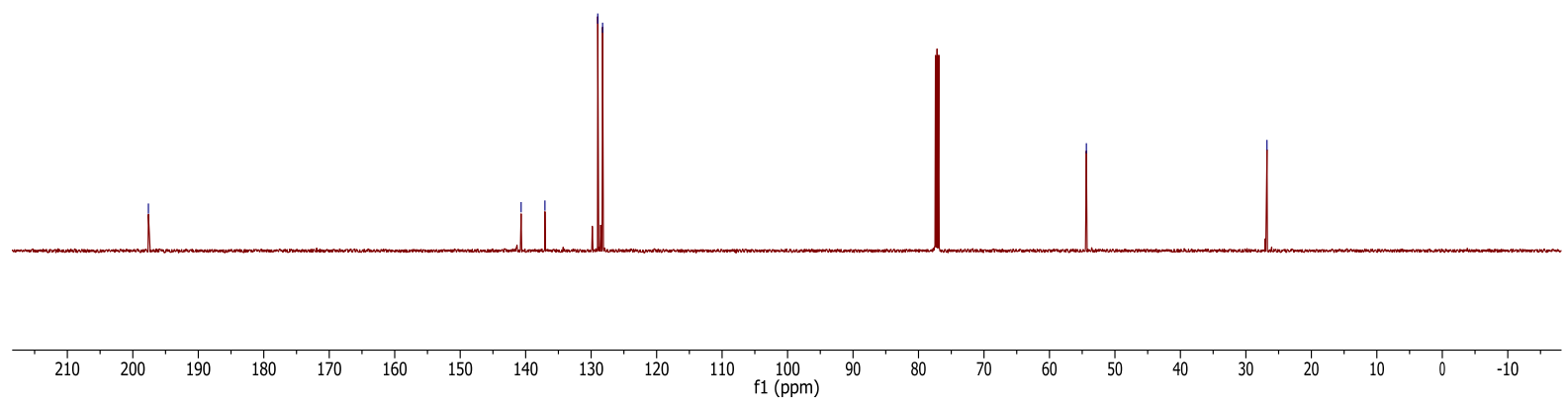

S32 
$3 q$

1H NMR, 400 MHz, CDCl3

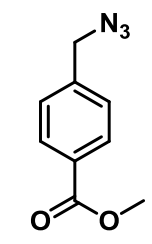

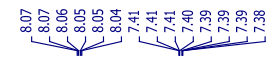
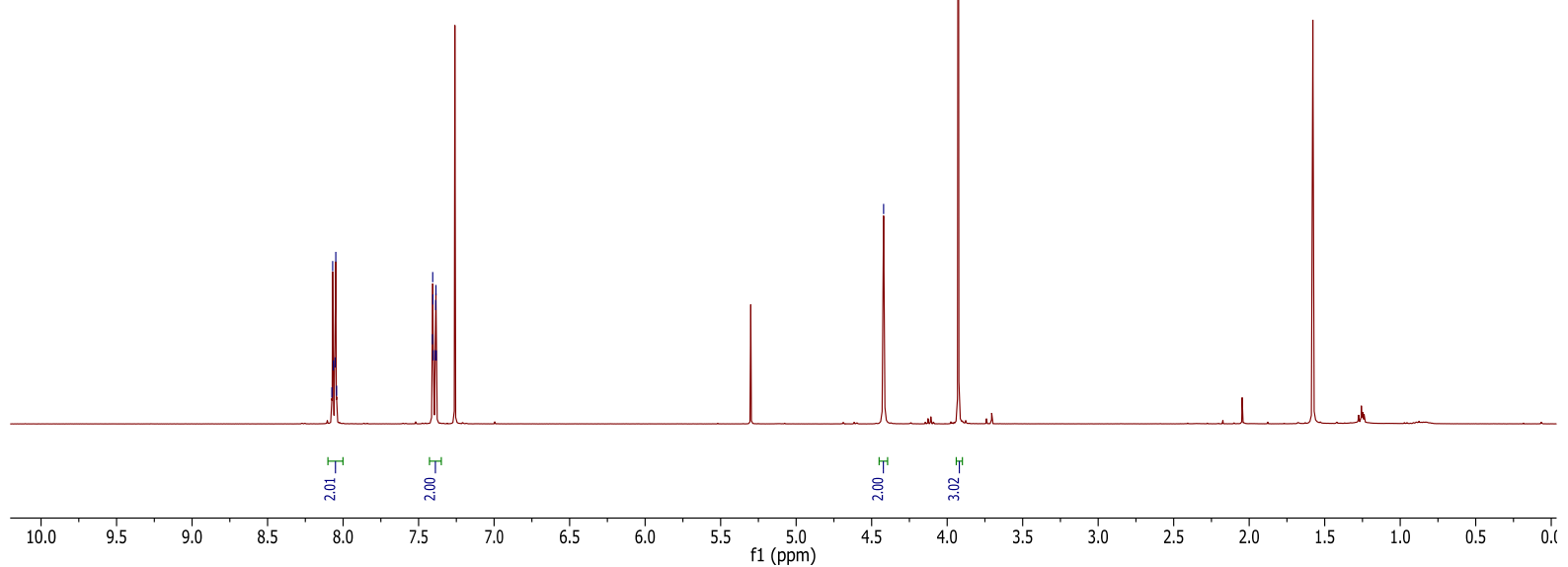

13C NMR, $100 \mathrm{MHz}, \mathrm{CDCl} 3$

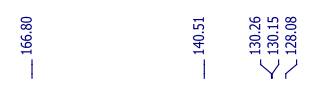

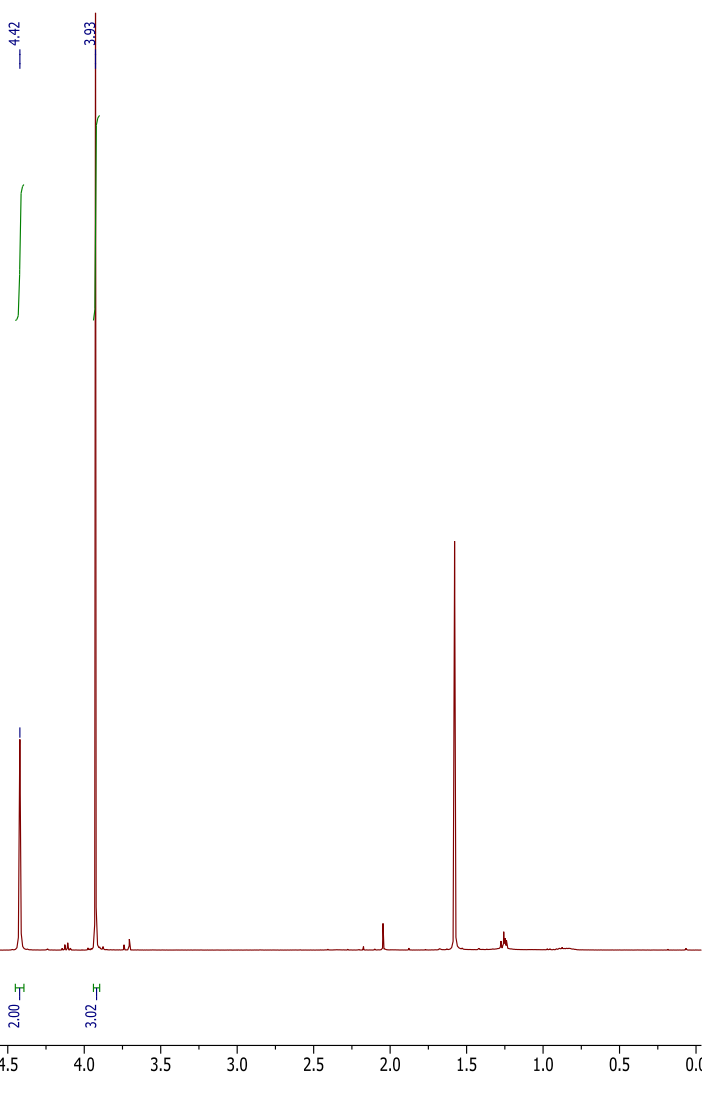

英是

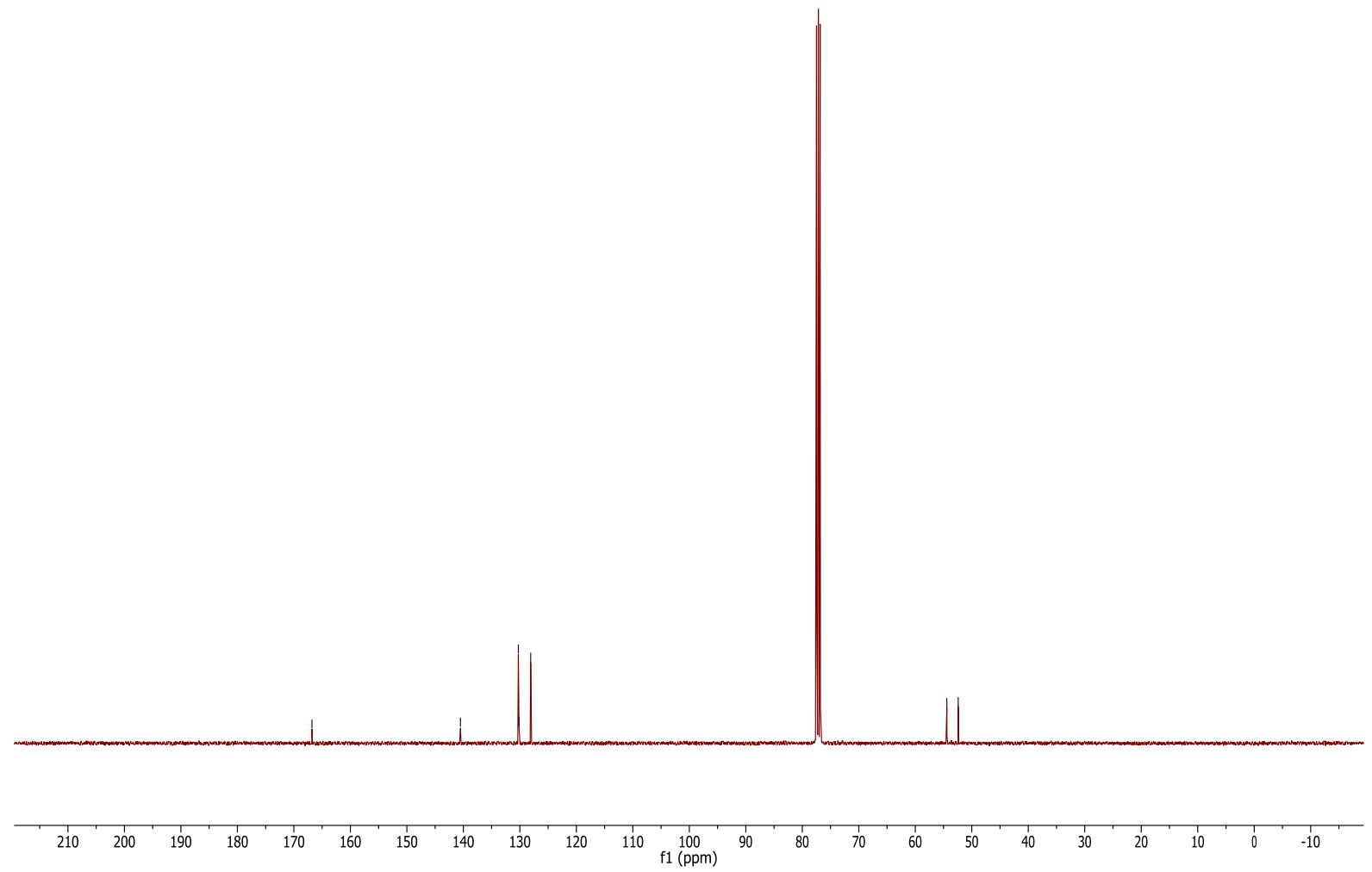

S33 
$3 \mathbf{r}$

$1 \mathrm{H} \mathrm{NMR,} 400 \mathrm{MHz}$, CDCl3<smiles>Cc1cc(Br)cc(CN)c1</smiles>
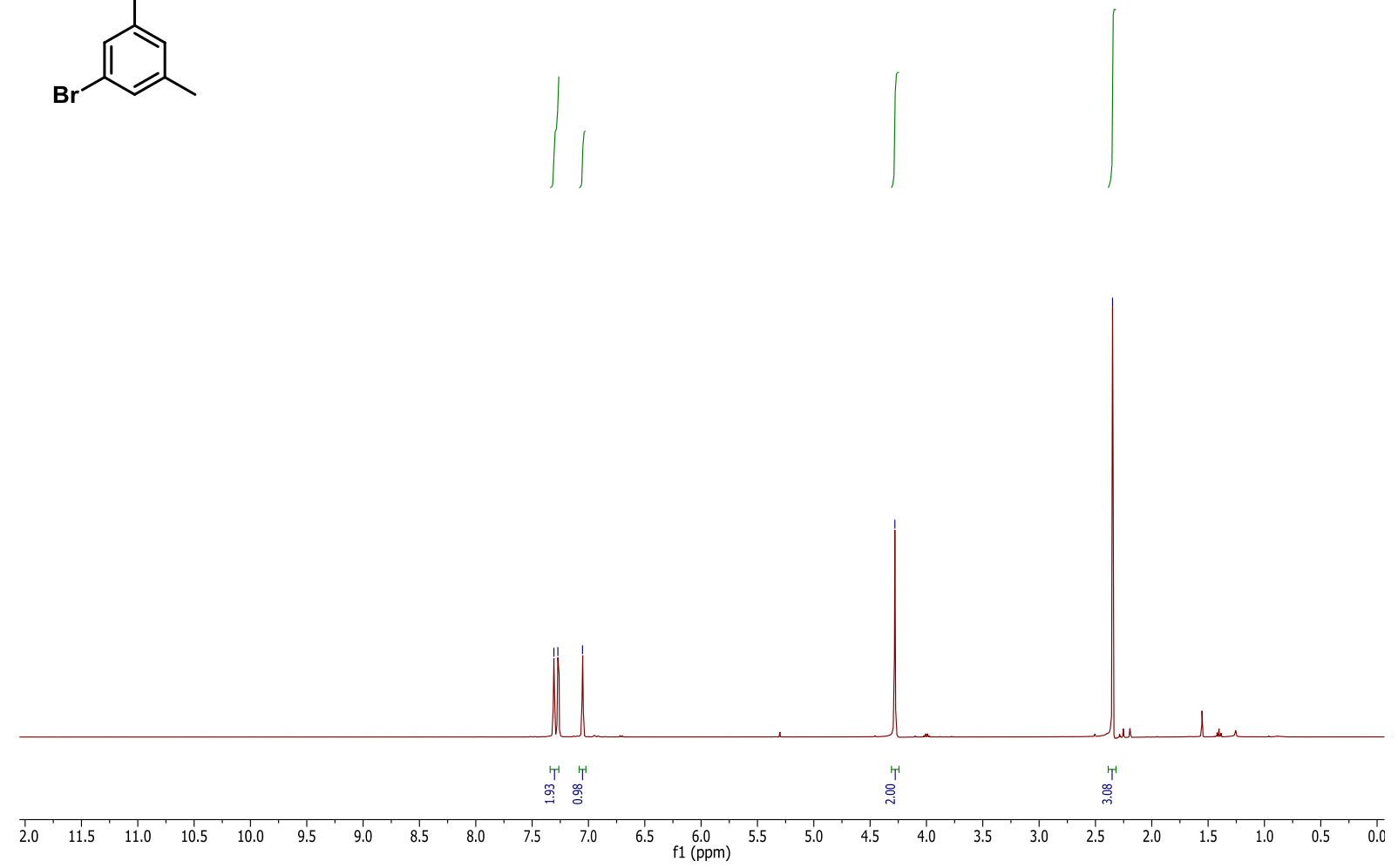

13C NMR, $101 \mathrm{MHz}, \mathrm{CDCl} 3$

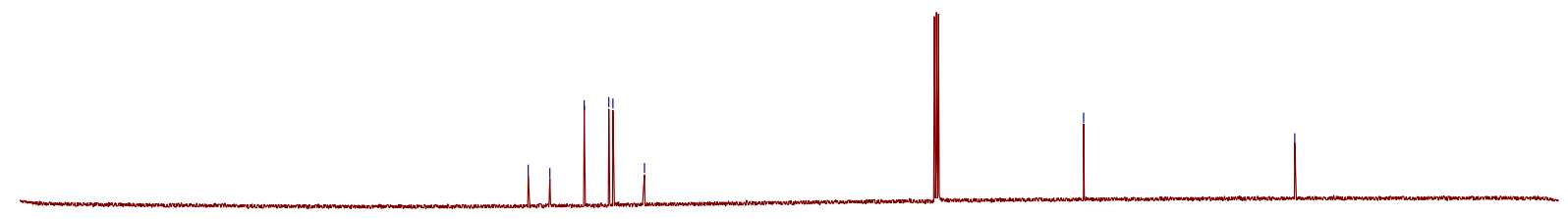

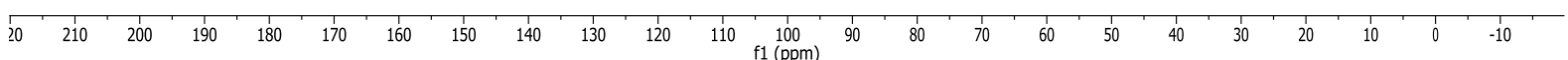


$3 s$

1H NMR, 400 MHz, CDCl3

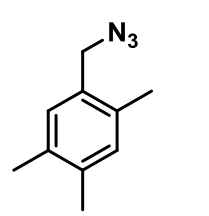

รูำ
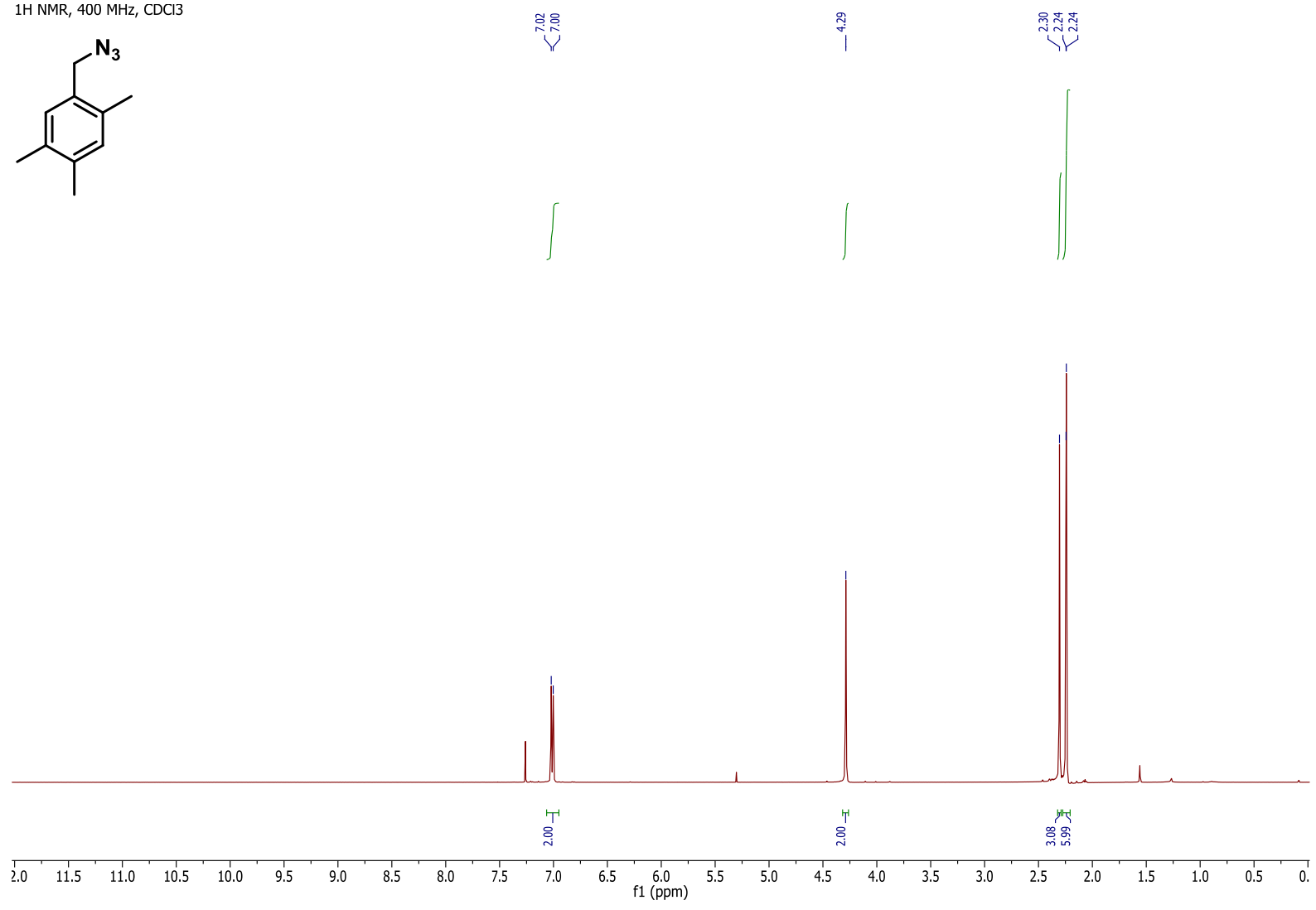

13C NMR, $101 \mathrm{MHz}, \mathrm{CDCl} 3$

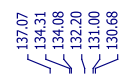

ตึำ

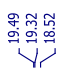
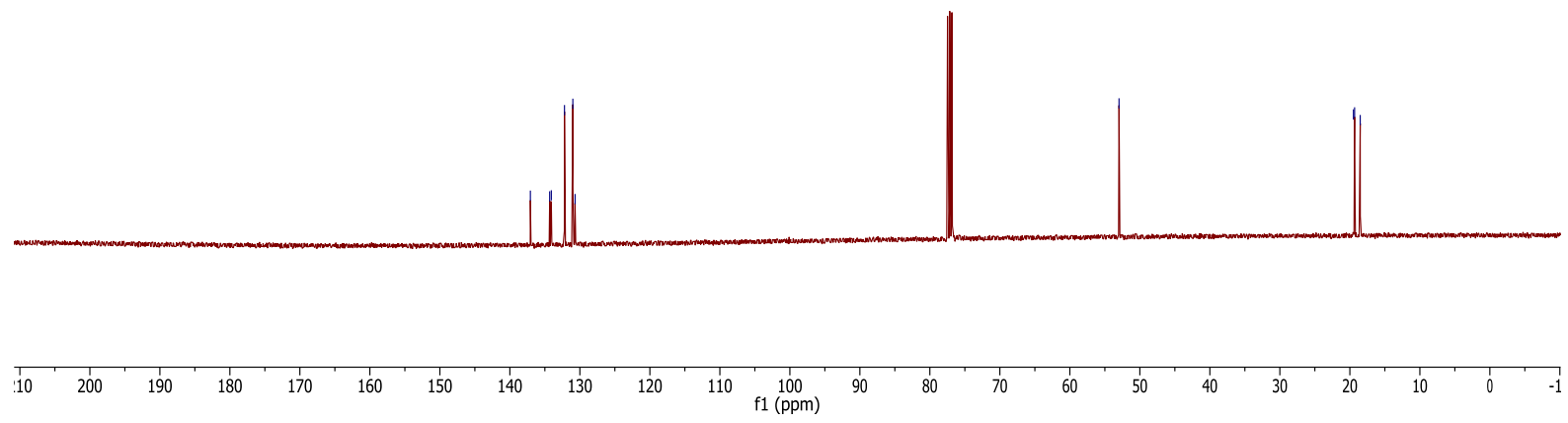

S35 
$3 t$

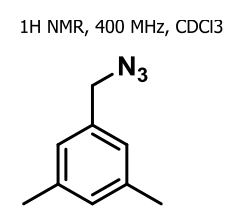

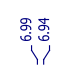

₹

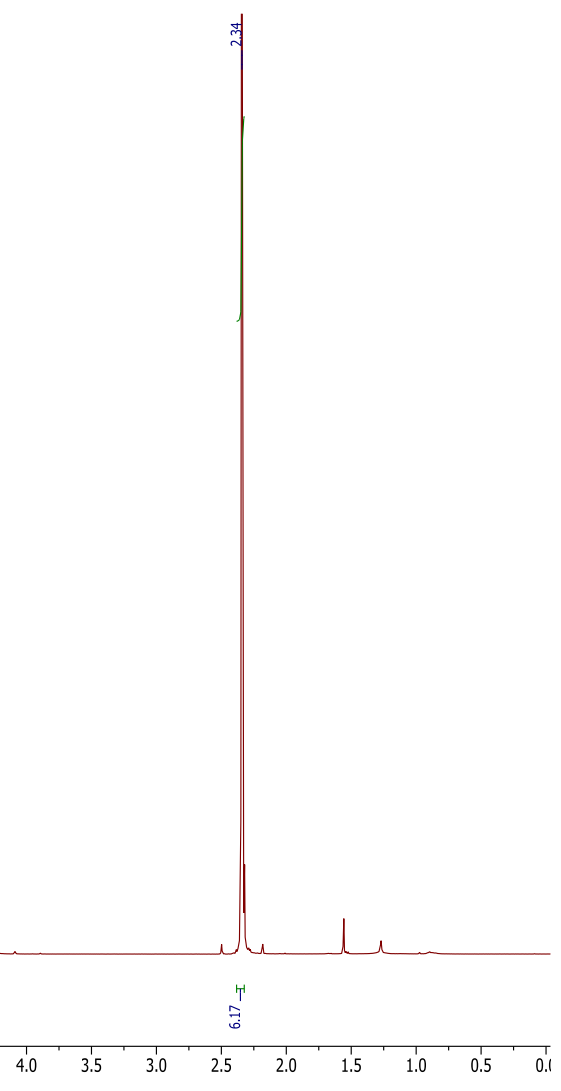

13C NMR, $101 \mathrm{MHz}, \mathrm{CDCl} 3$

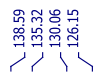

票

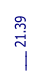

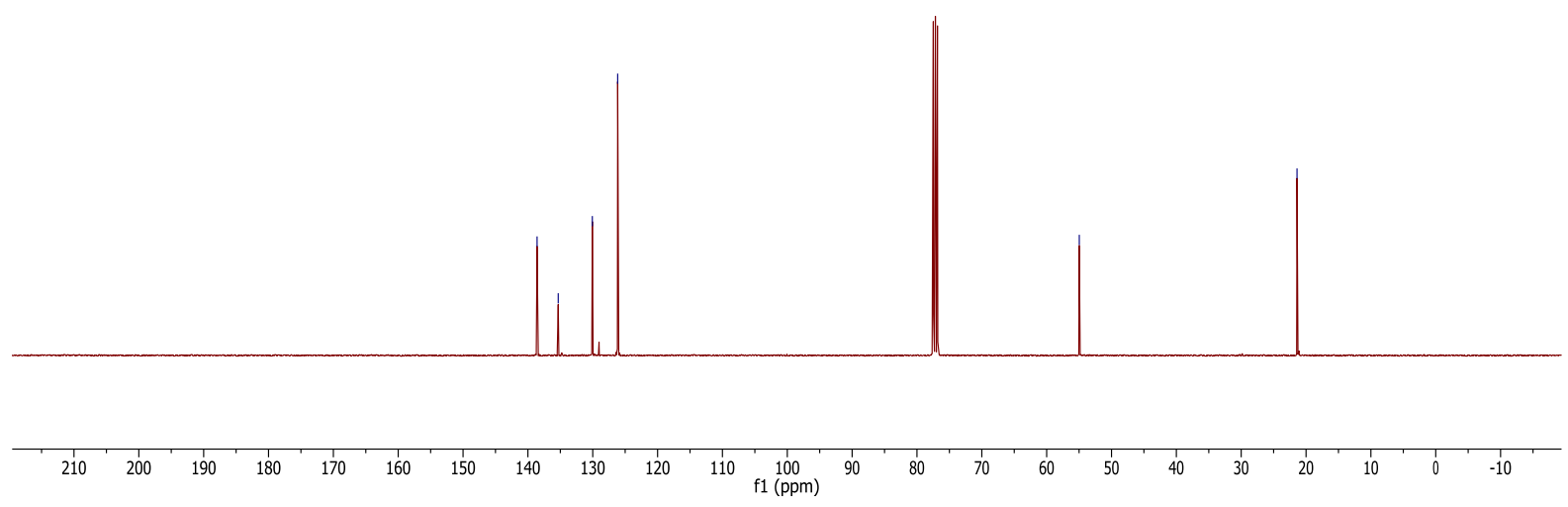

S36 
3u

1H NMR, 400 MHz, CDCl3
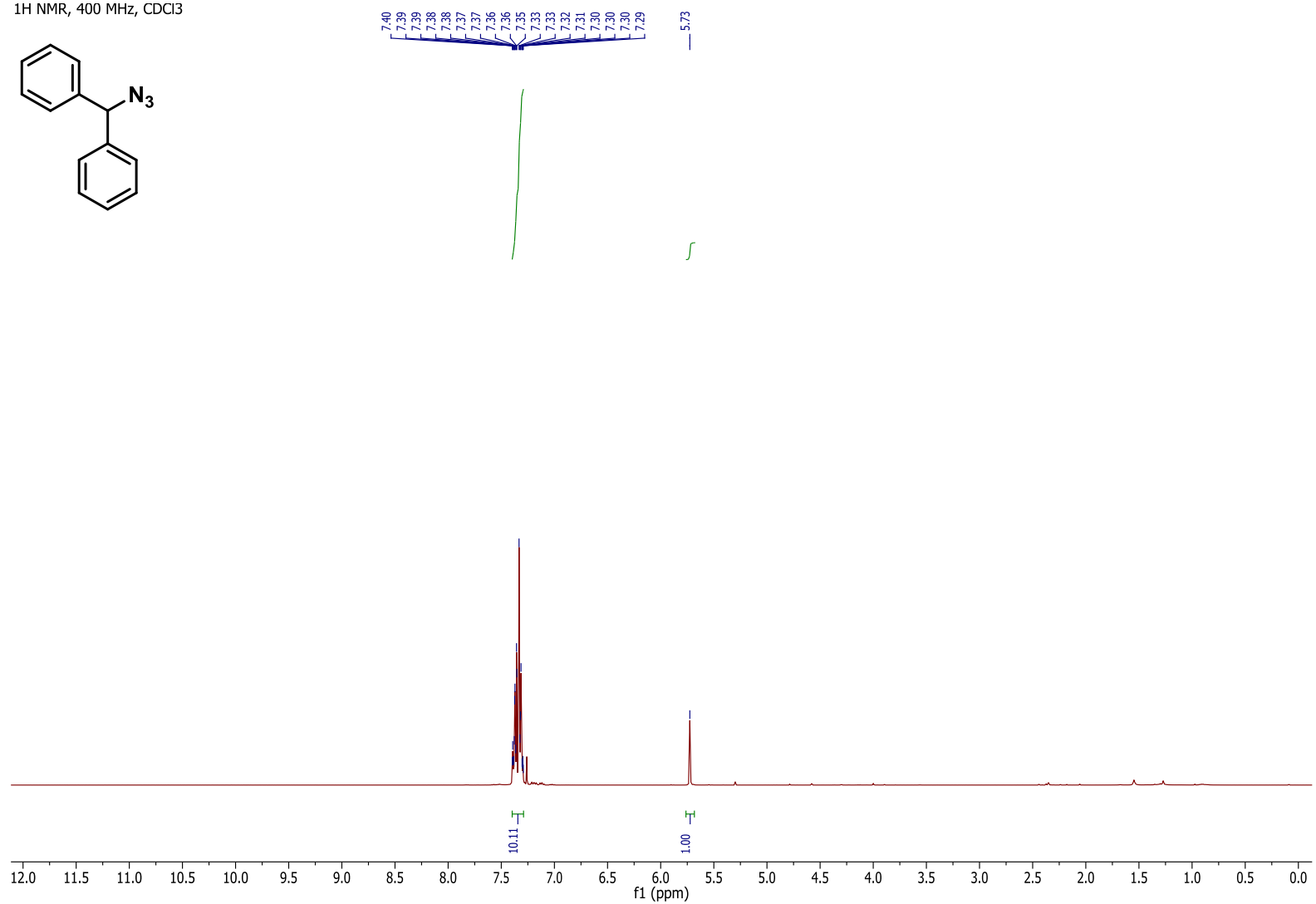

13C NMR, 101 MHz, CDCl3

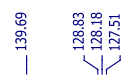

产

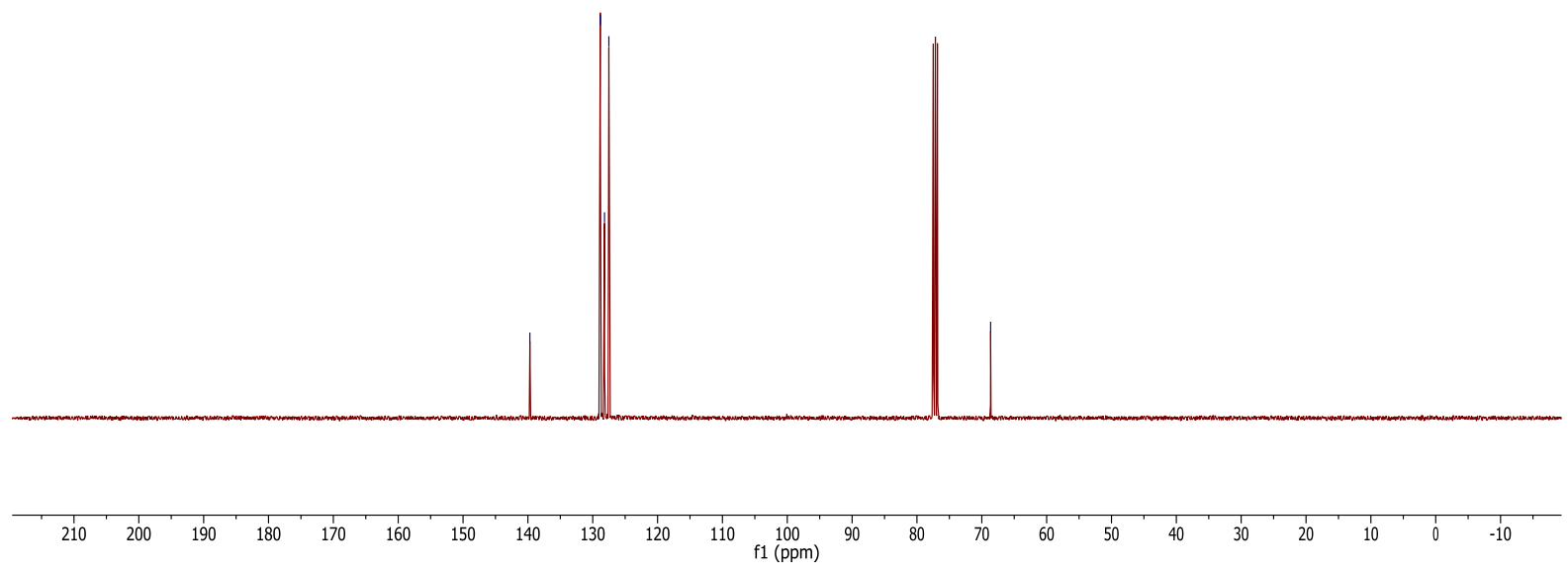

S37 
3v

1H NMR, $400 \mathrm{MHz}, \mathrm{CDCl} 3$

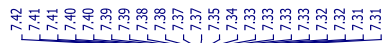

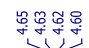

量离
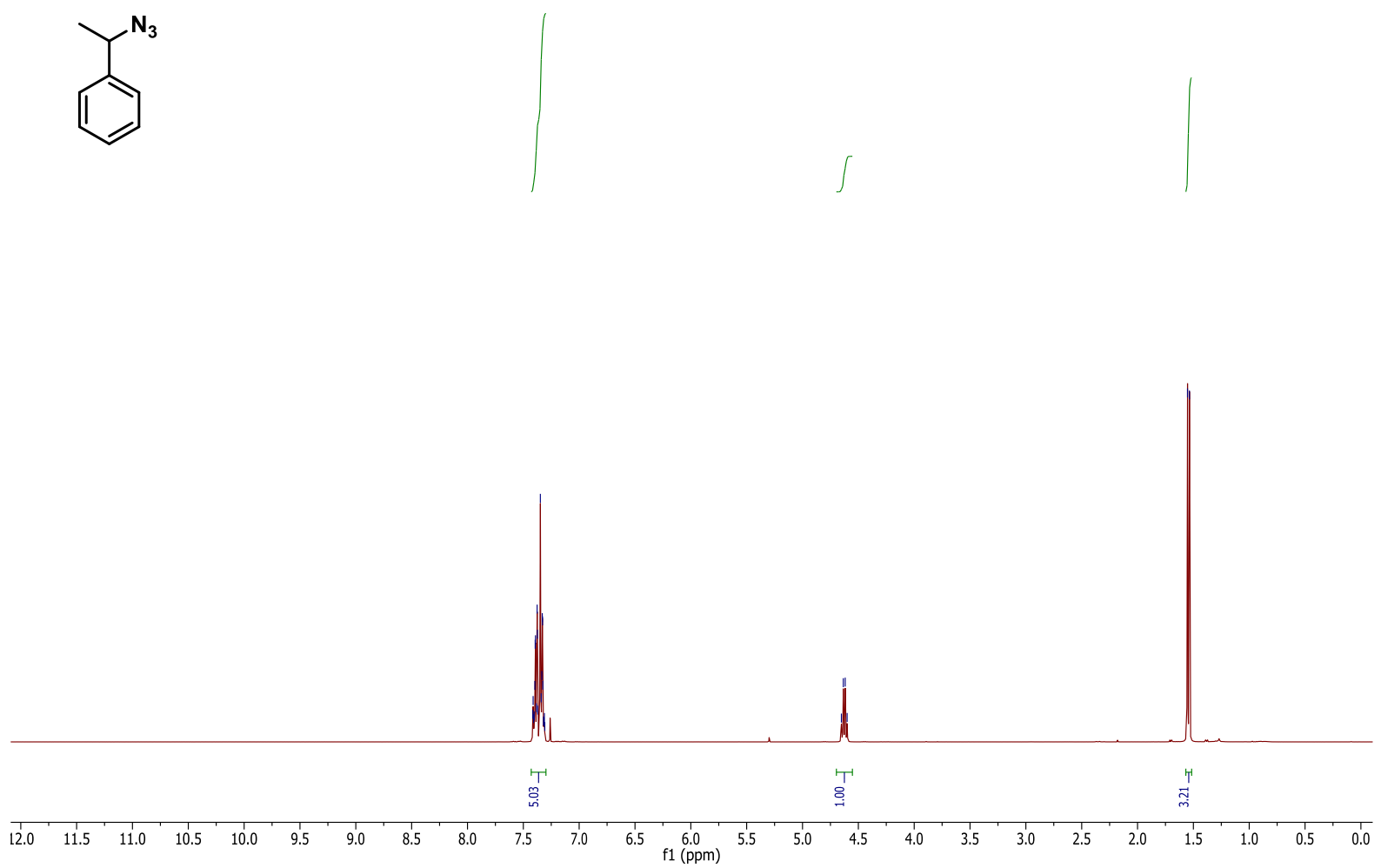

13C NMR, $101 \mathrm{MHz}$, CDCl3

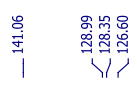

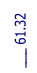

$\stackrel{\infty}{\stackrel{\infty}{*}}$

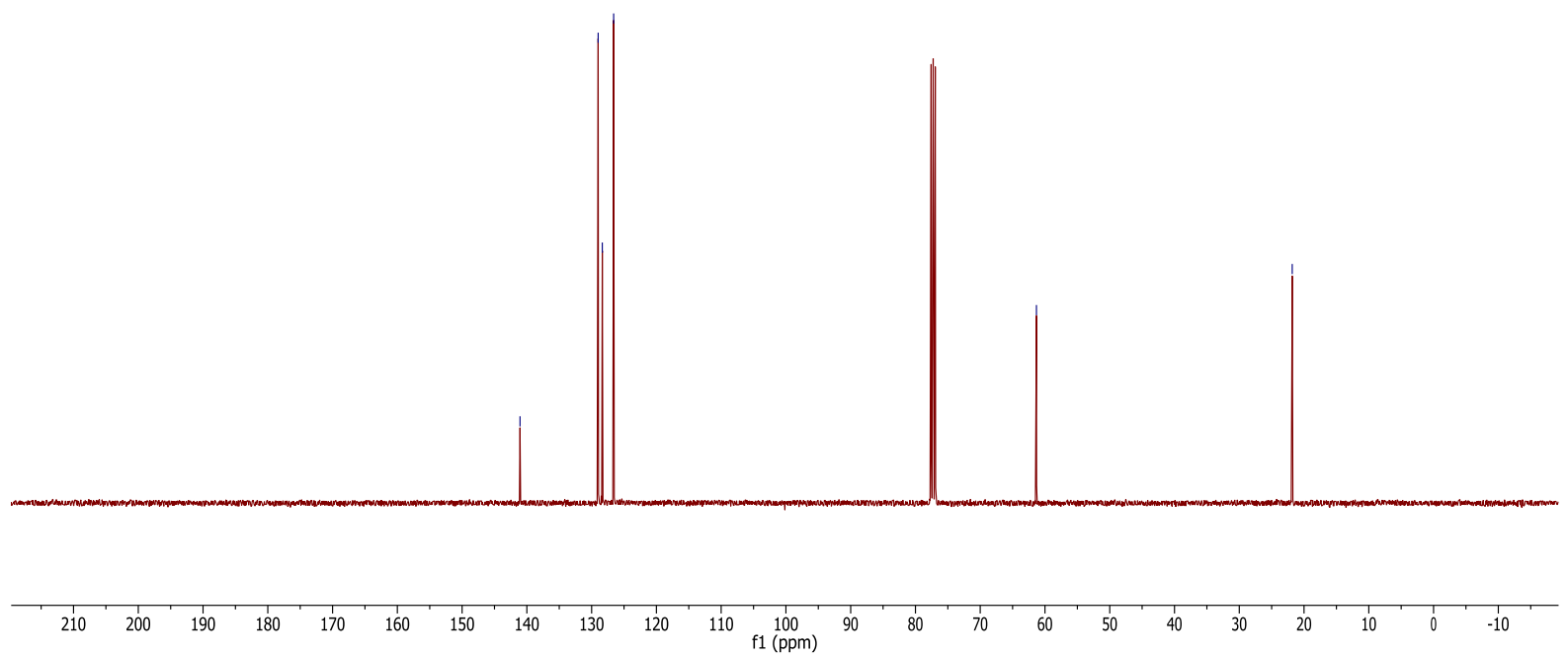

S38 
3w

$1 \mathrm{H} \mathrm{NMR,} 400 \mathrm{MHz}, \mathrm{CDCl} 3$

좄 종

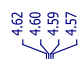

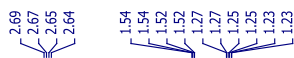<smiles>CCc1ccc(C(C)N)cc1</smiles>
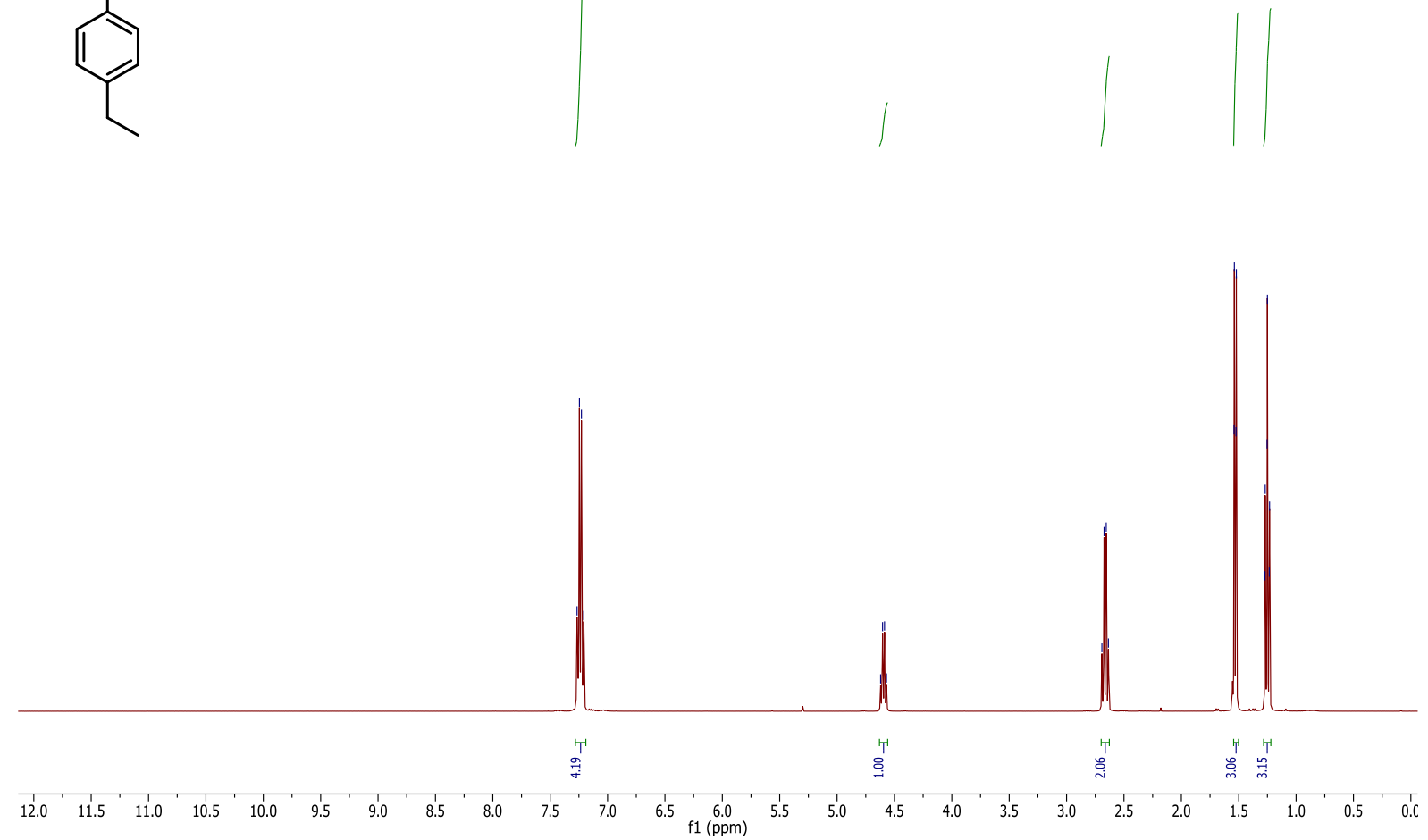

13C NMR, 101 MHz, CDCl3

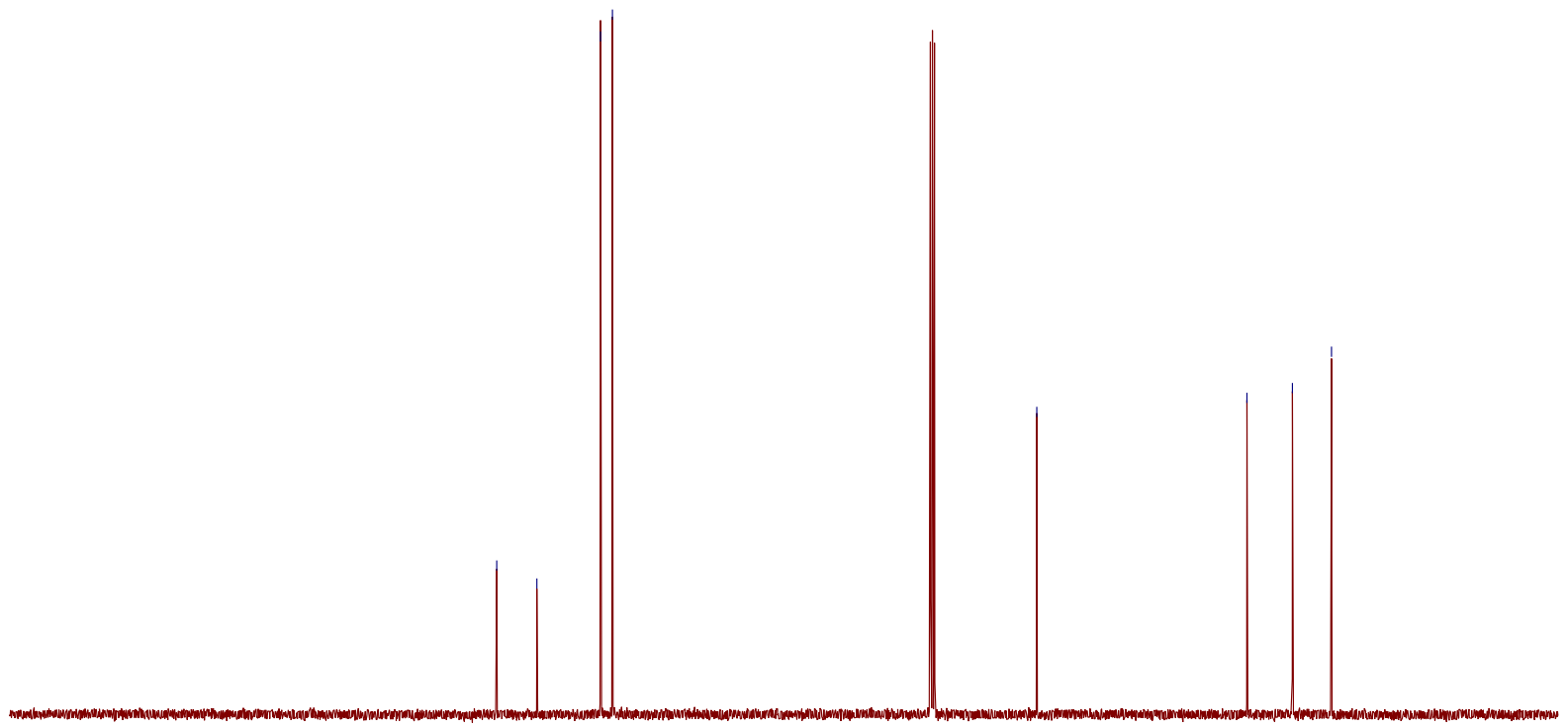


$3 \mathbf{x}$

$1 \mathrm{H} \mathrm{NMR,} 400 \mathrm{MHz}, \mathrm{CDCl} 3$<smiles>CC(C)c1cc(C(C)C)cc(C(C)(C)N)c1</smiles>

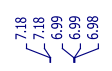

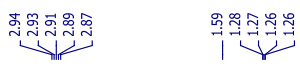

Is
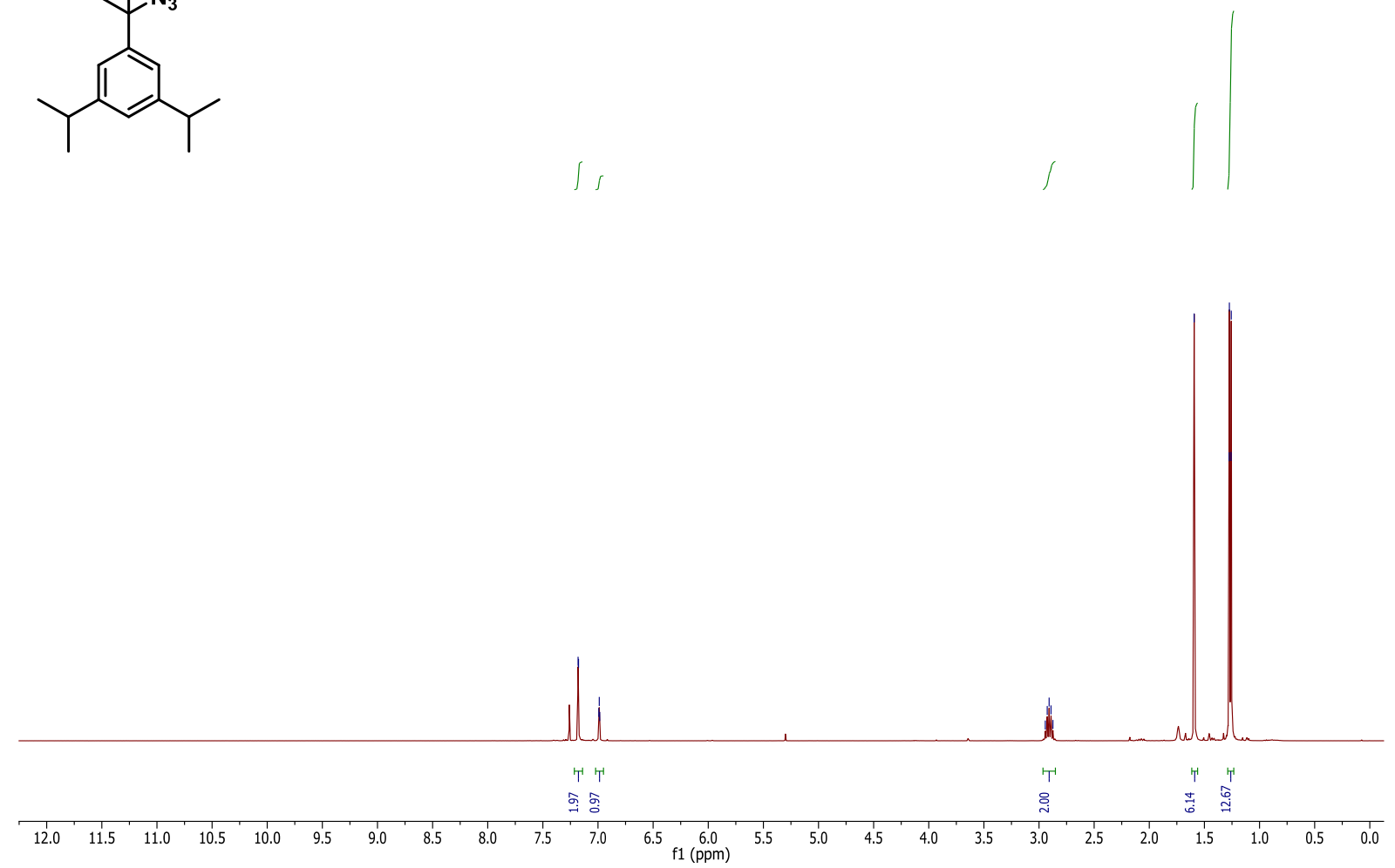

13C NMR, $101 \mathrm{MHz}, \mathrm{CDCl} 3$

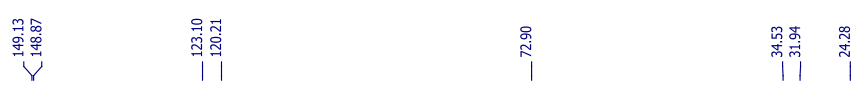

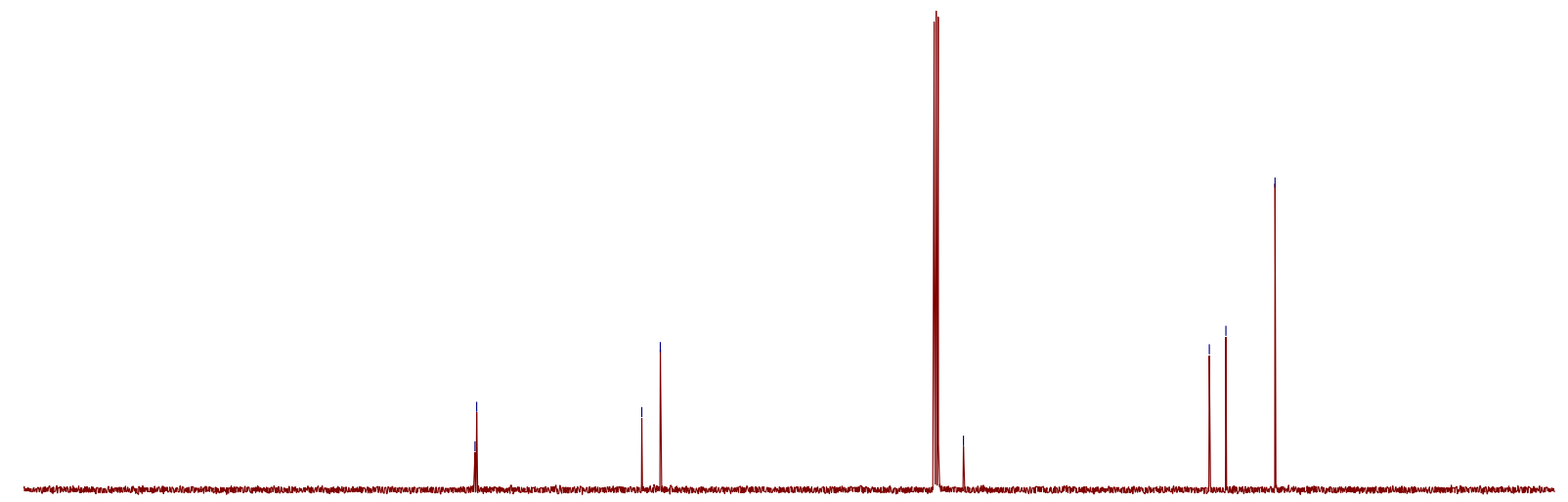


$3 y$

$1 \mathrm{H} \mathrm{NMR}, 500 \mathrm{MHz}, \mathrm{CDCl}$<smiles>Cc1ccc(C(C)(C)C)cc1</smiles>

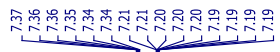

$\tilde{*}$

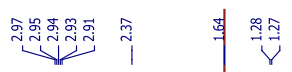

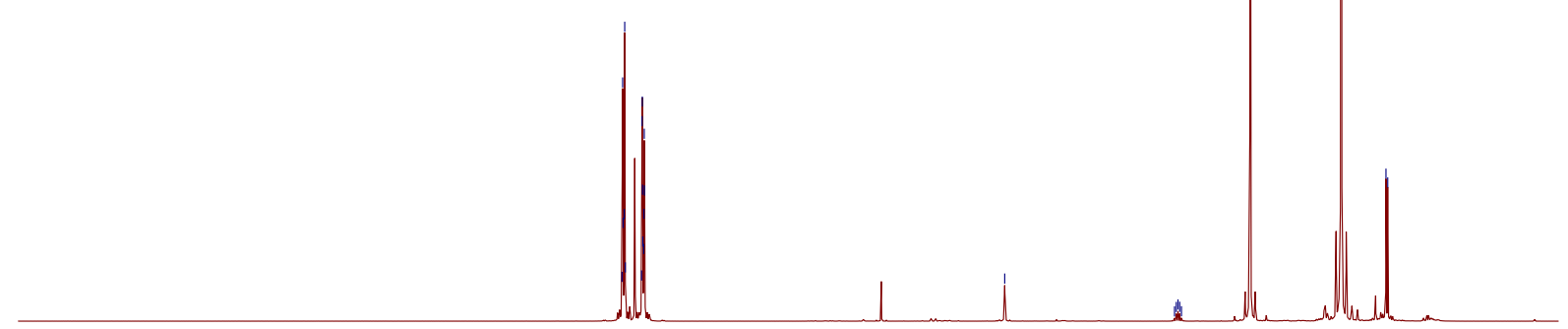

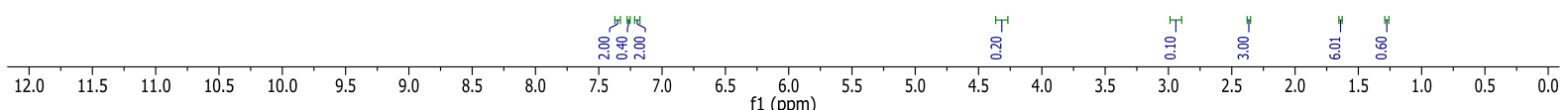

13C NMR, 126 MHz, CDCl3

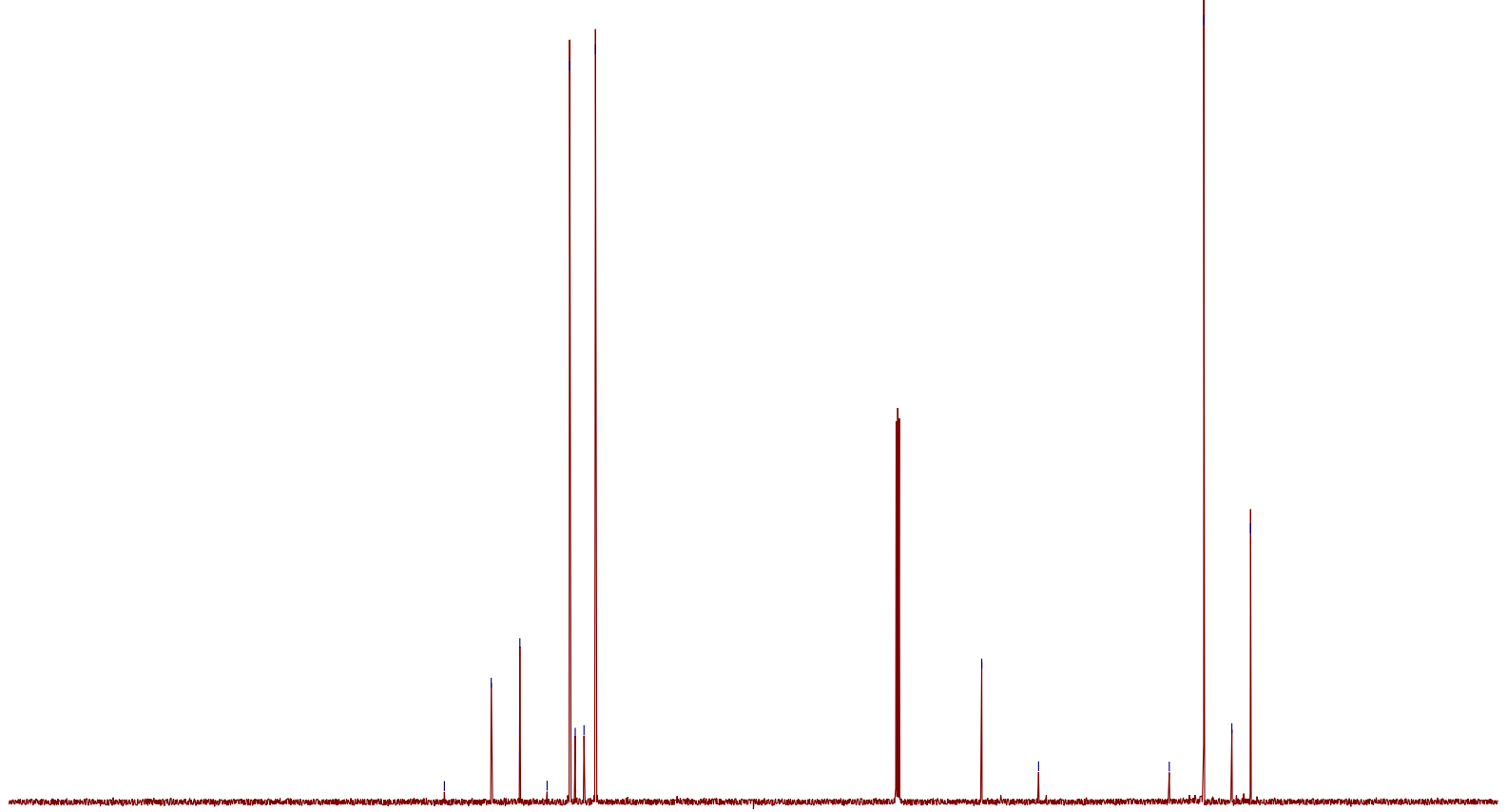

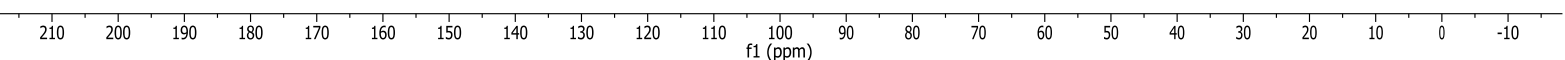


$1 \mathrm{HNMR}, 400 \mathrm{MHz}$, CDCl3<smiles>CC(C)(C)OC(=O)n1c(CN)cc2ccccc21</smiles>

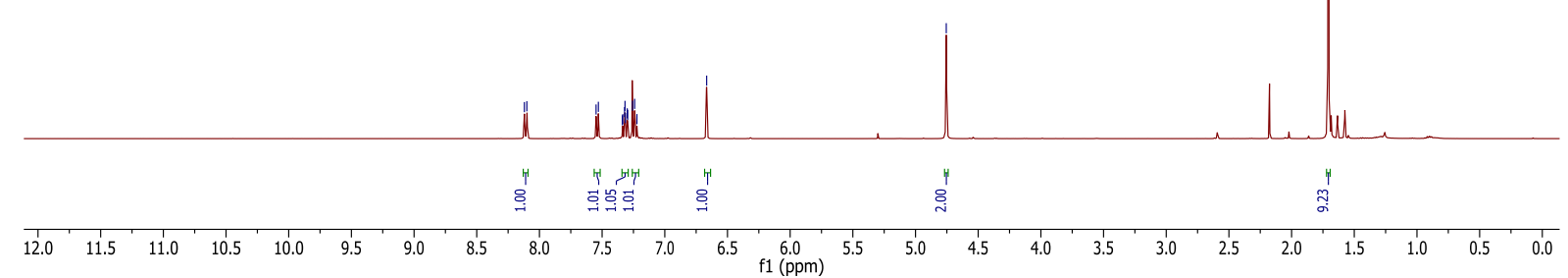

13C NMR, 101 MHz, CDCl3

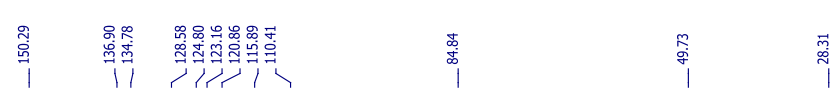

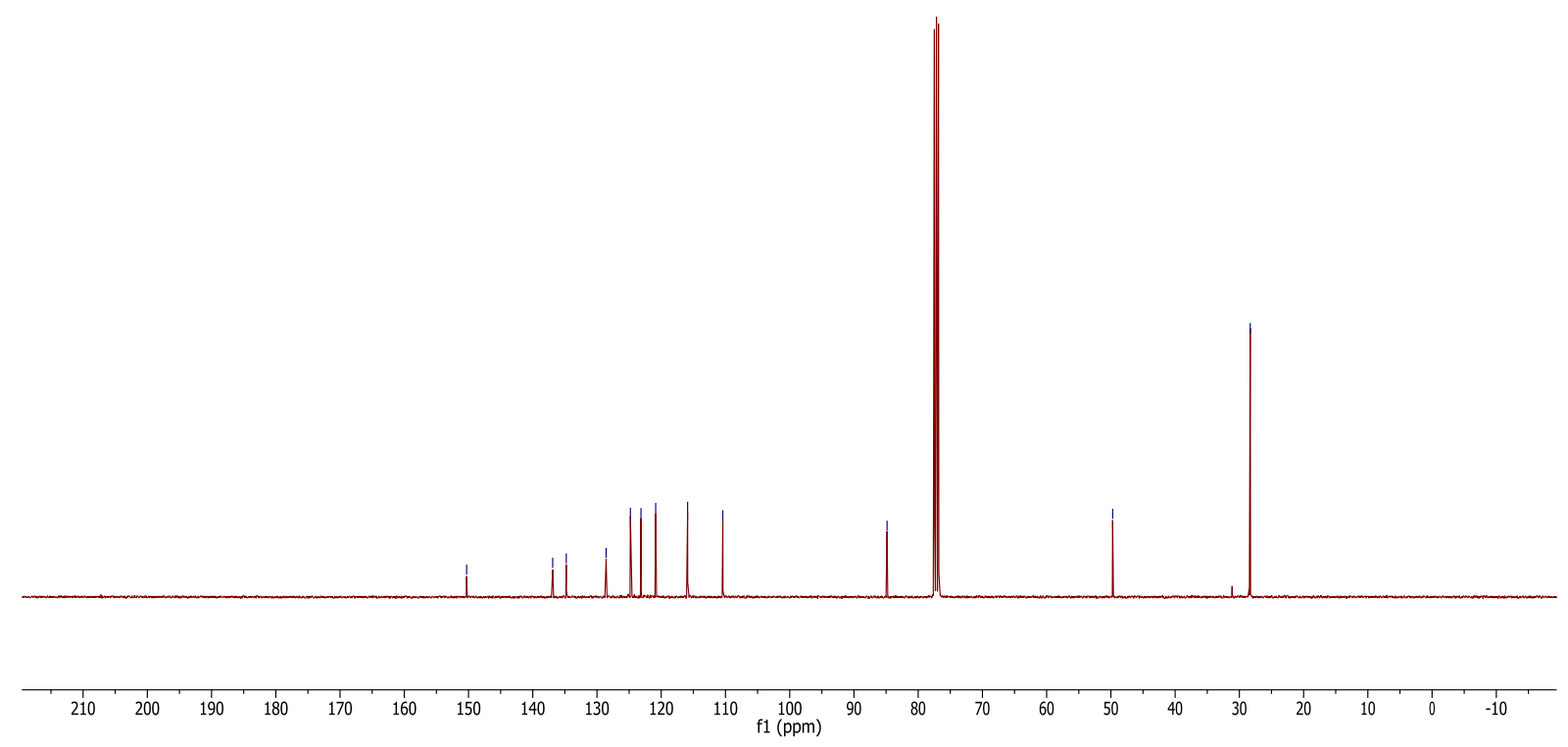


3bb

1H NMR, $400 \mathrm{MHz}, \mathrm{CDCl} 3$

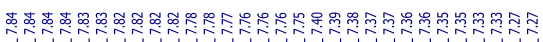
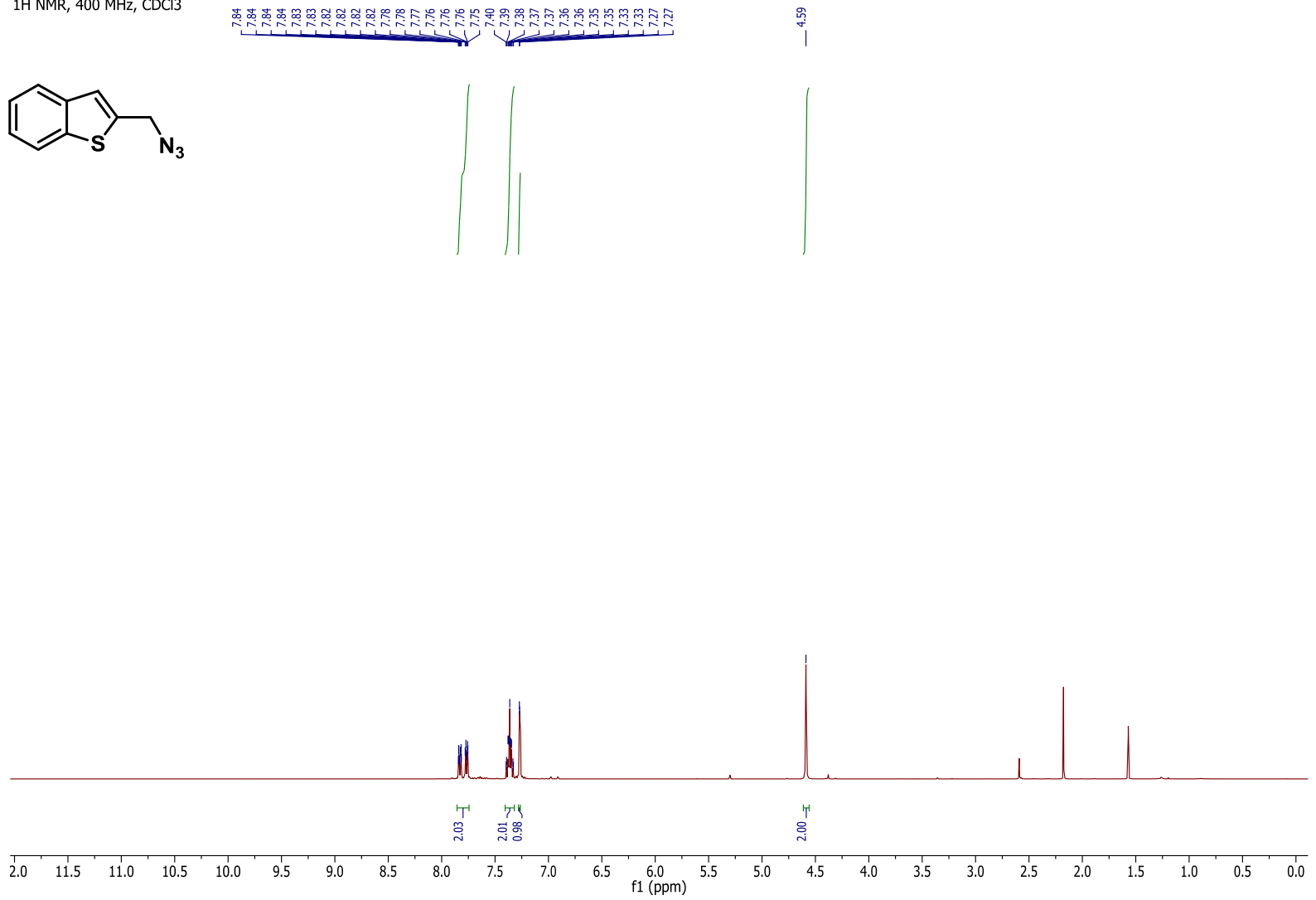

13C NMR, $101 \mathrm{MHz}, \mathrm{CDCl} 3$

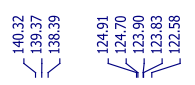

$\stackrel{m}{\stackrel{m}{\circ} !}$

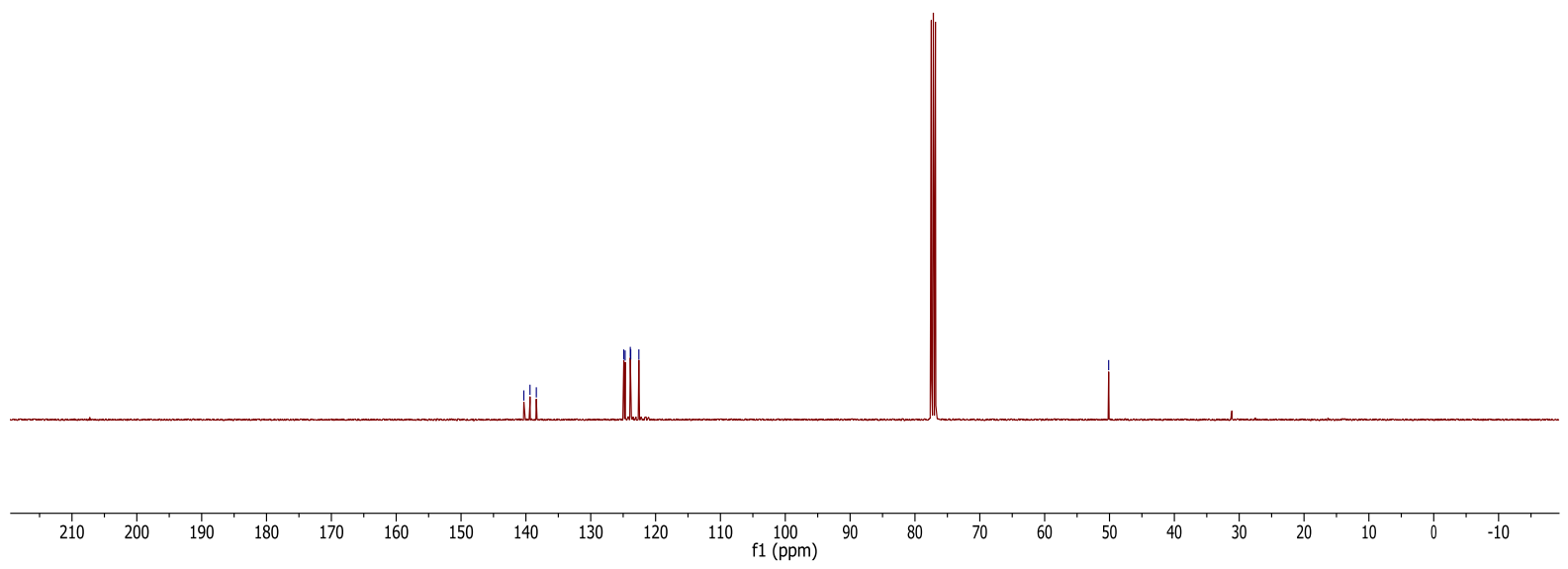

S43 
$3 \mathrm{cc}$

$1 \mathrm{H} \mathrm{NMR,} 500$ MHz, CDCl3<smiles>Cc1ccc(SCN)cc1</smiles>
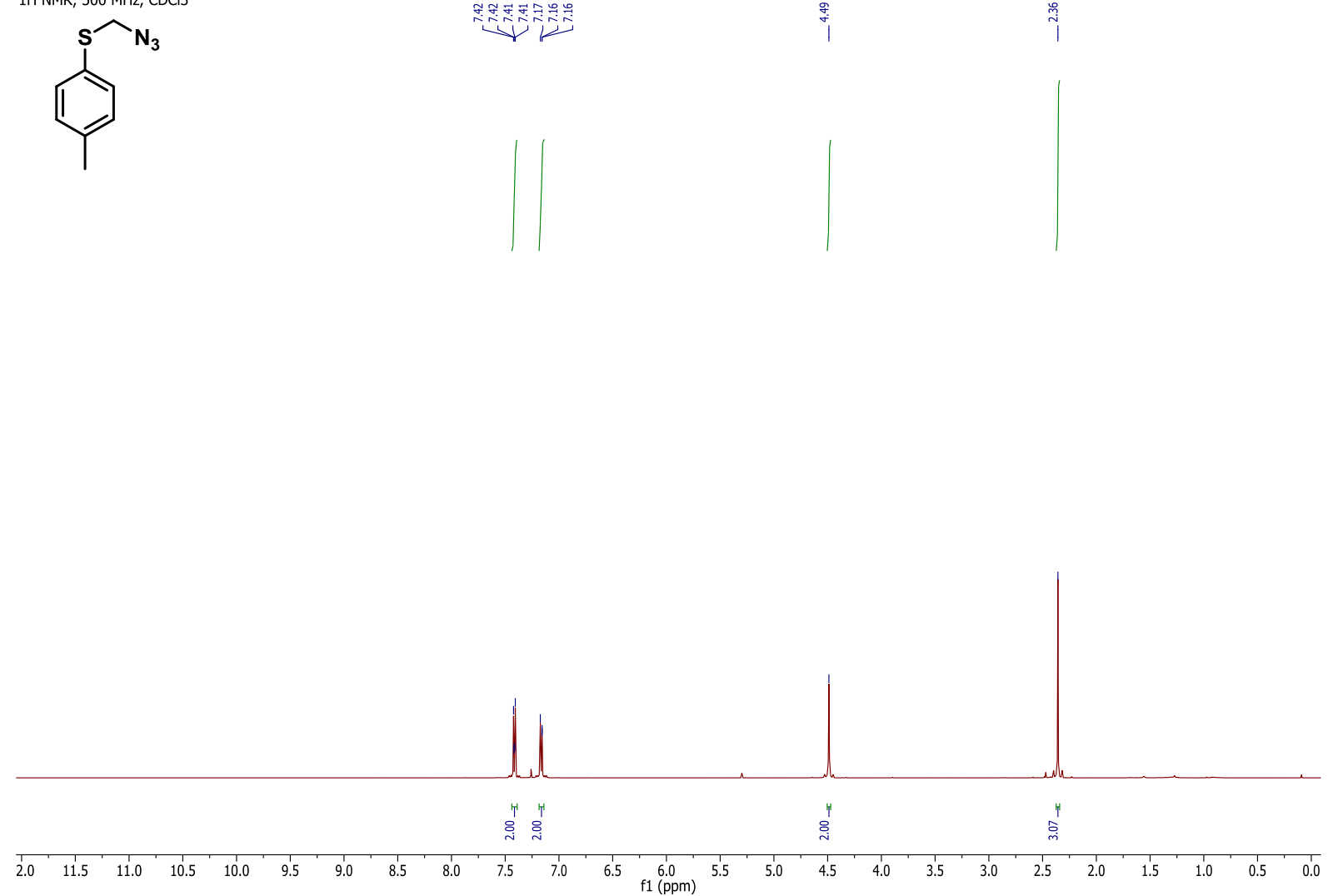

13C NMR, $126 \mathrm{MHz}, \mathrm{CDCl} 3$

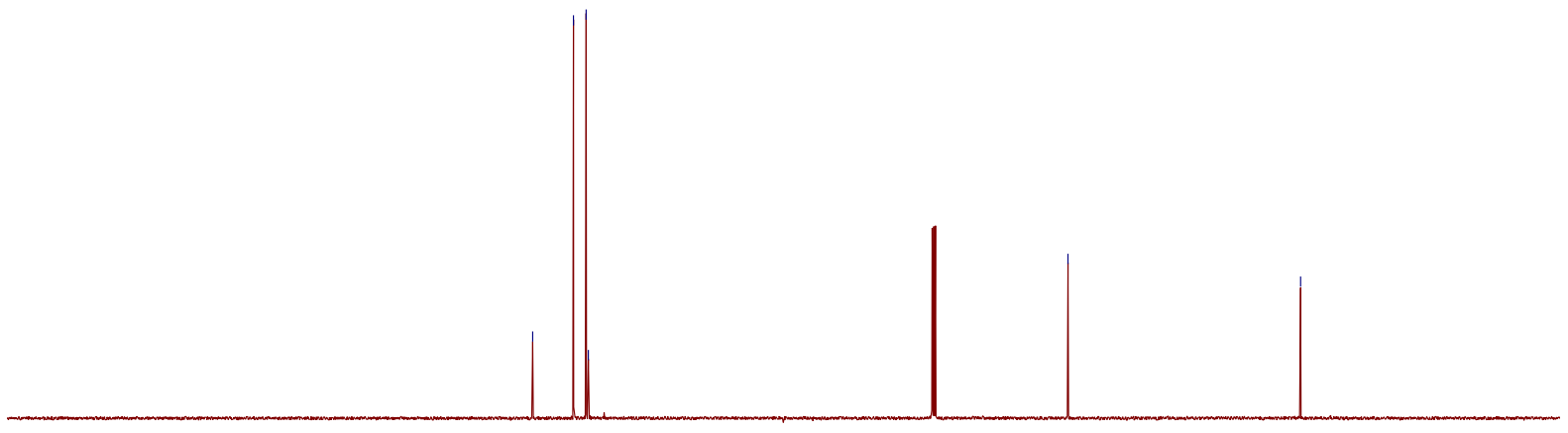

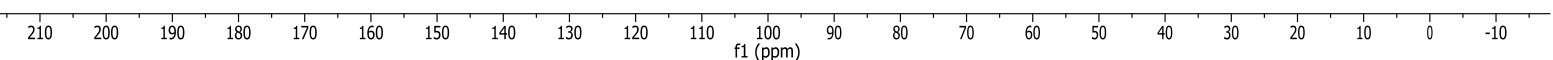


3dd

$1 \mathrm{H} \mathrm{NMR,} 400 \mathrm{MHz}, \mathrm{CDCl} 3$

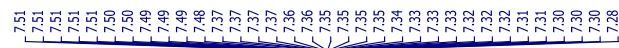
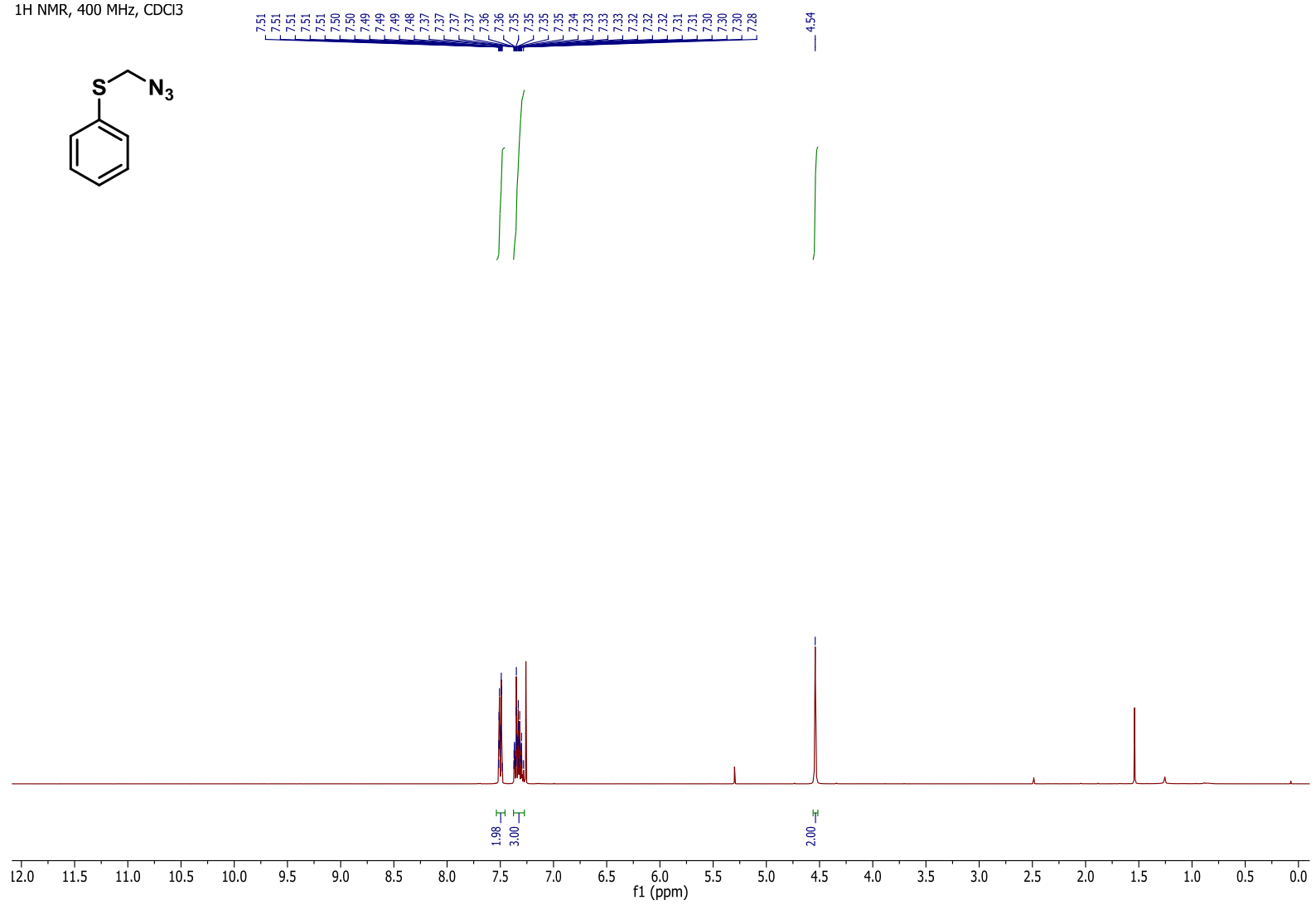

13C NMR, $101 \mathrm{MHz}, \mathrm{CDCl} 3$

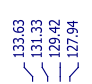

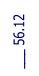

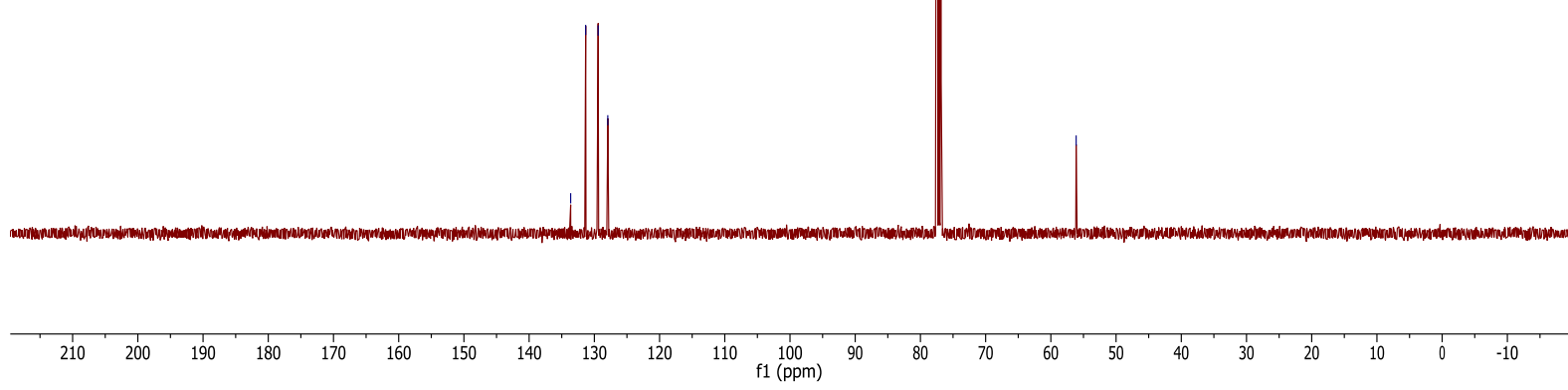

S45 
3ee

$1 \mathrm{H} \mathrm{NMR,} 400$ MHz, CDCl3<smiles>NCSc1ccc(Br)cc1</smiles>

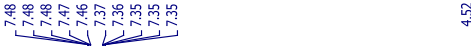
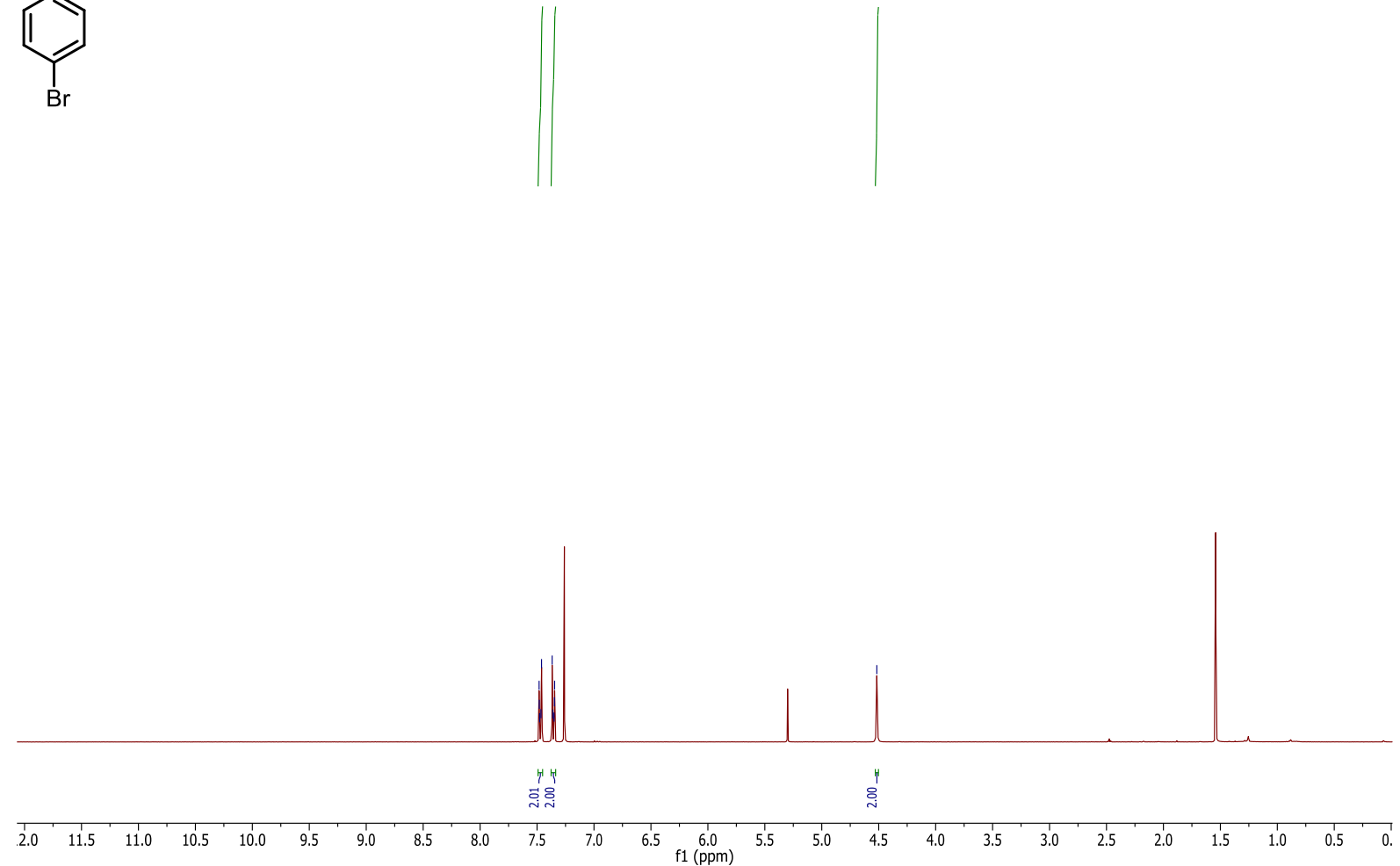

C13 NMR, 101 MHz, CDCl3

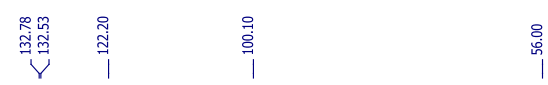

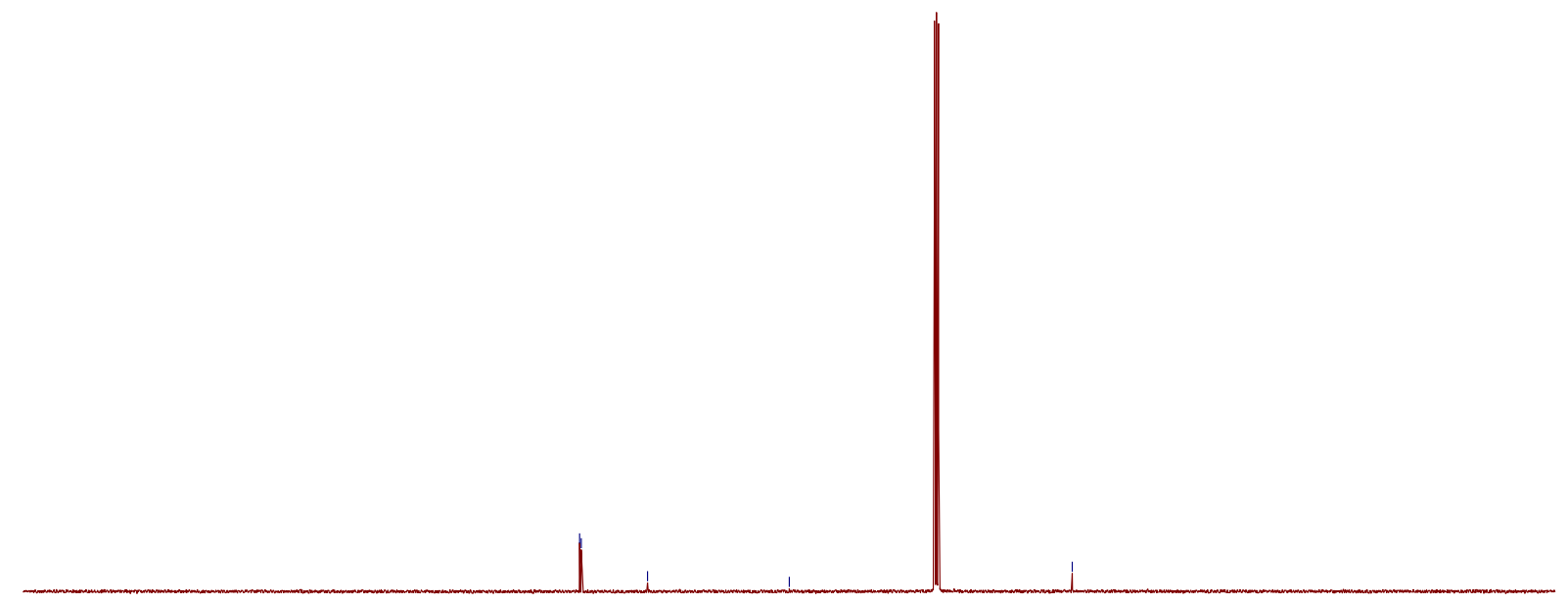

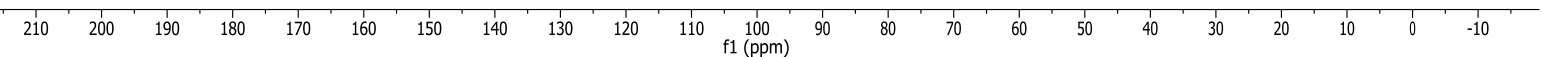


3ff

$1 \mathrm{H} \mathrm{NMR,} 500 \mathrm{MHz}, \mathrm{CDCl} 3$<smiles>N#Cc1c(F)cccc1F</smiles>

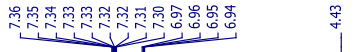

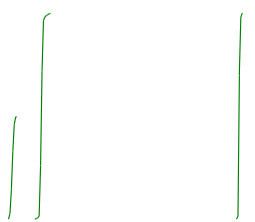

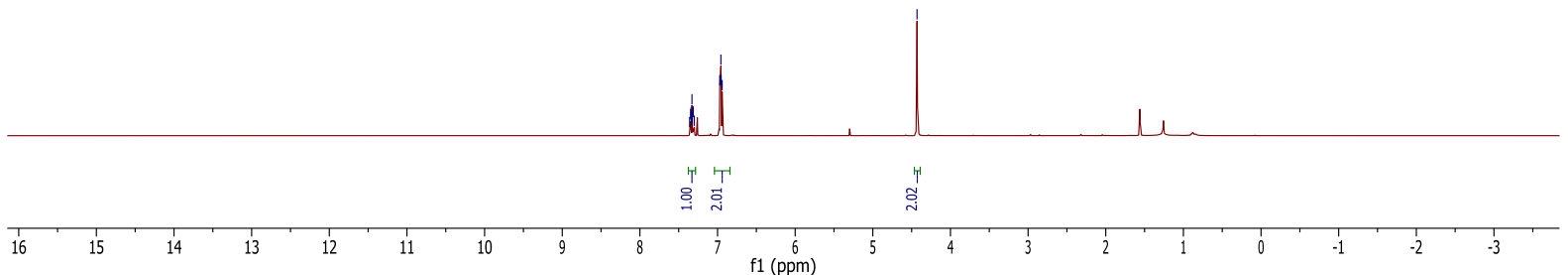

13C NMR, 126 MHz, CDCl3

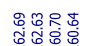

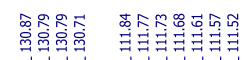

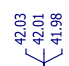

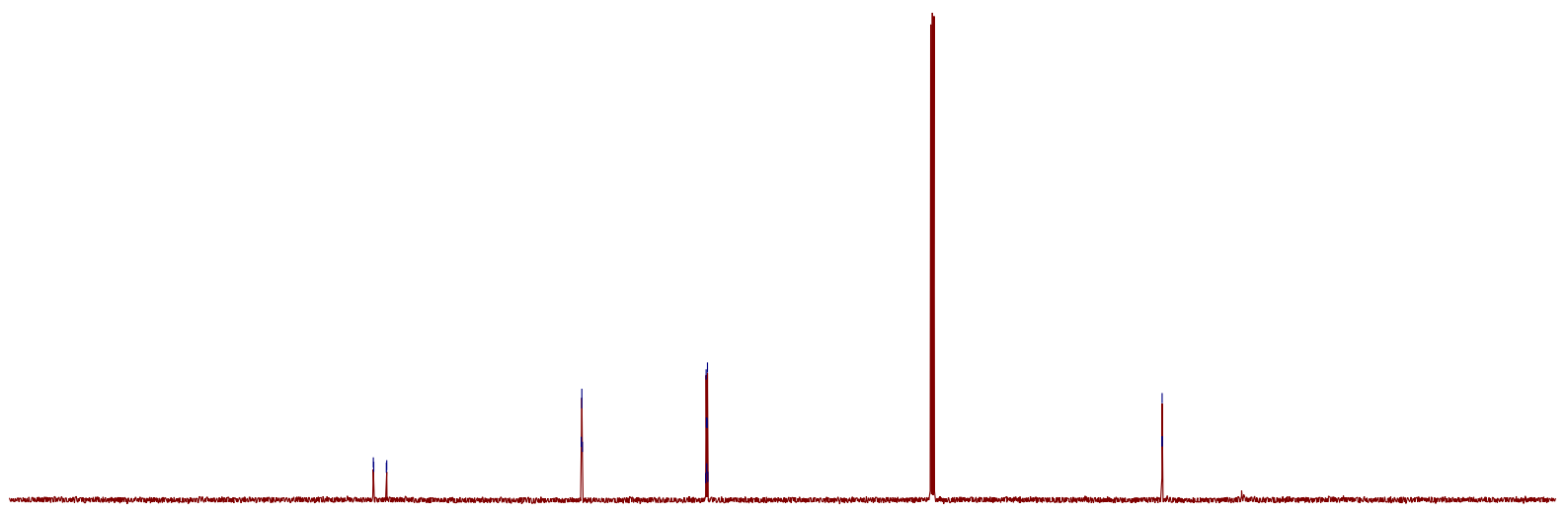

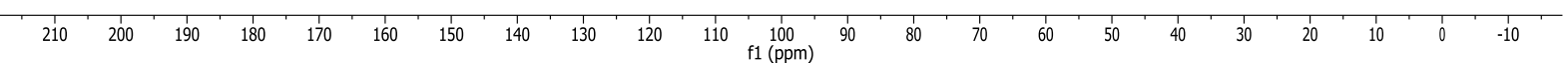




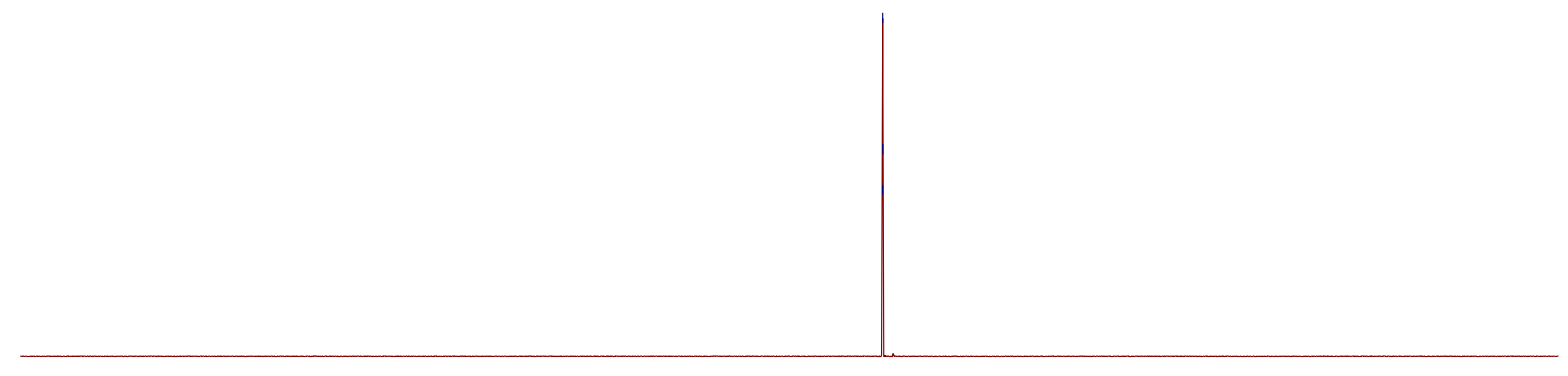

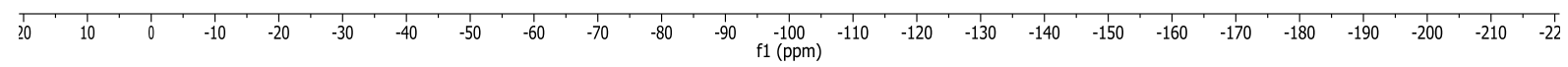
4

$1 \mathrm{H} \mathrm{NMR,}, 400 \mathrm{MHz}$, (CD3)2SO<smiles>NC(=O)c1cn(Cc2c(F)cccc2F)nn1</smiles>

|
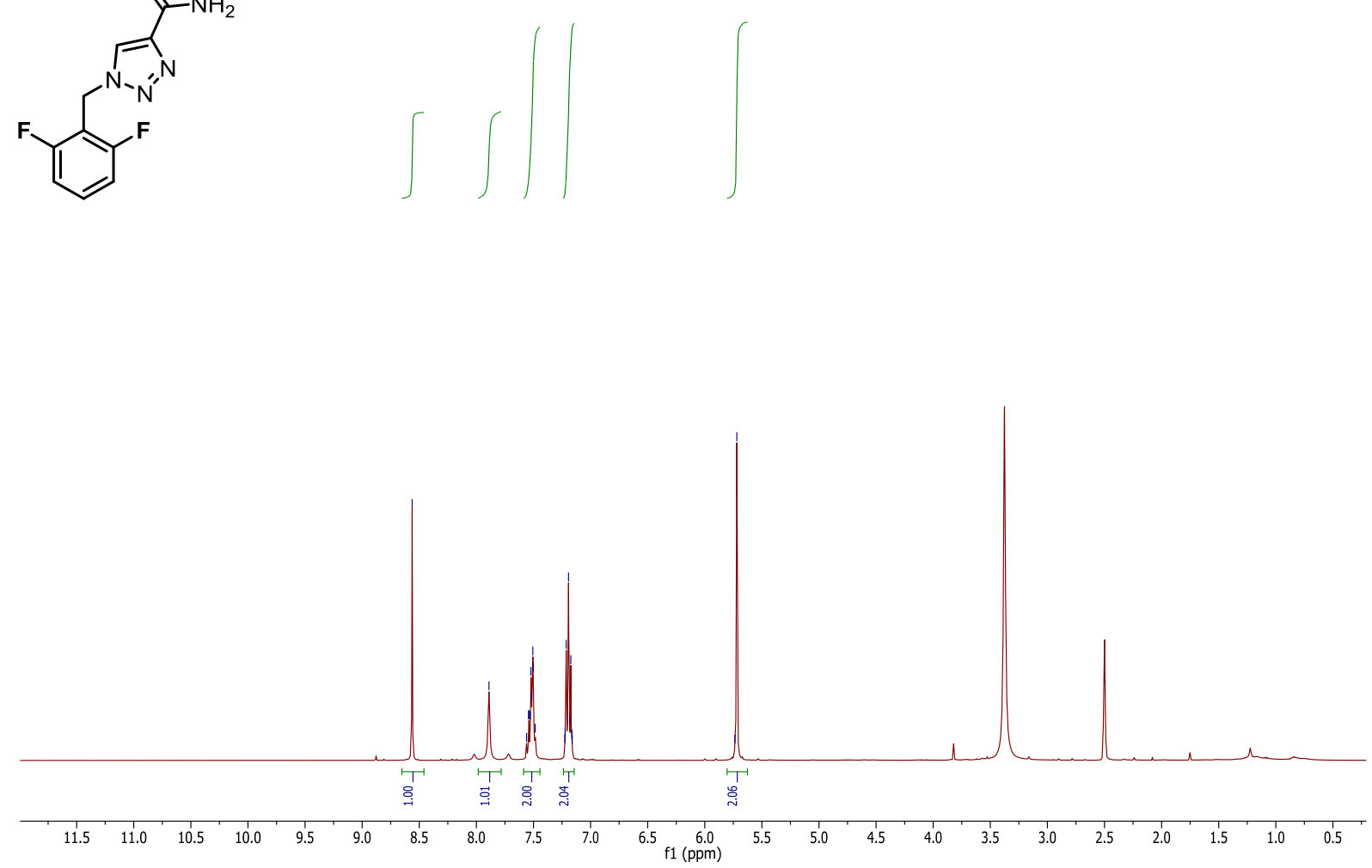
4

13C NMR, $100 \mathrm{MHz}$, (CD3)2SO

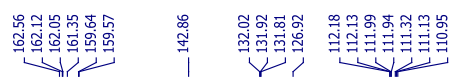

翠䍂

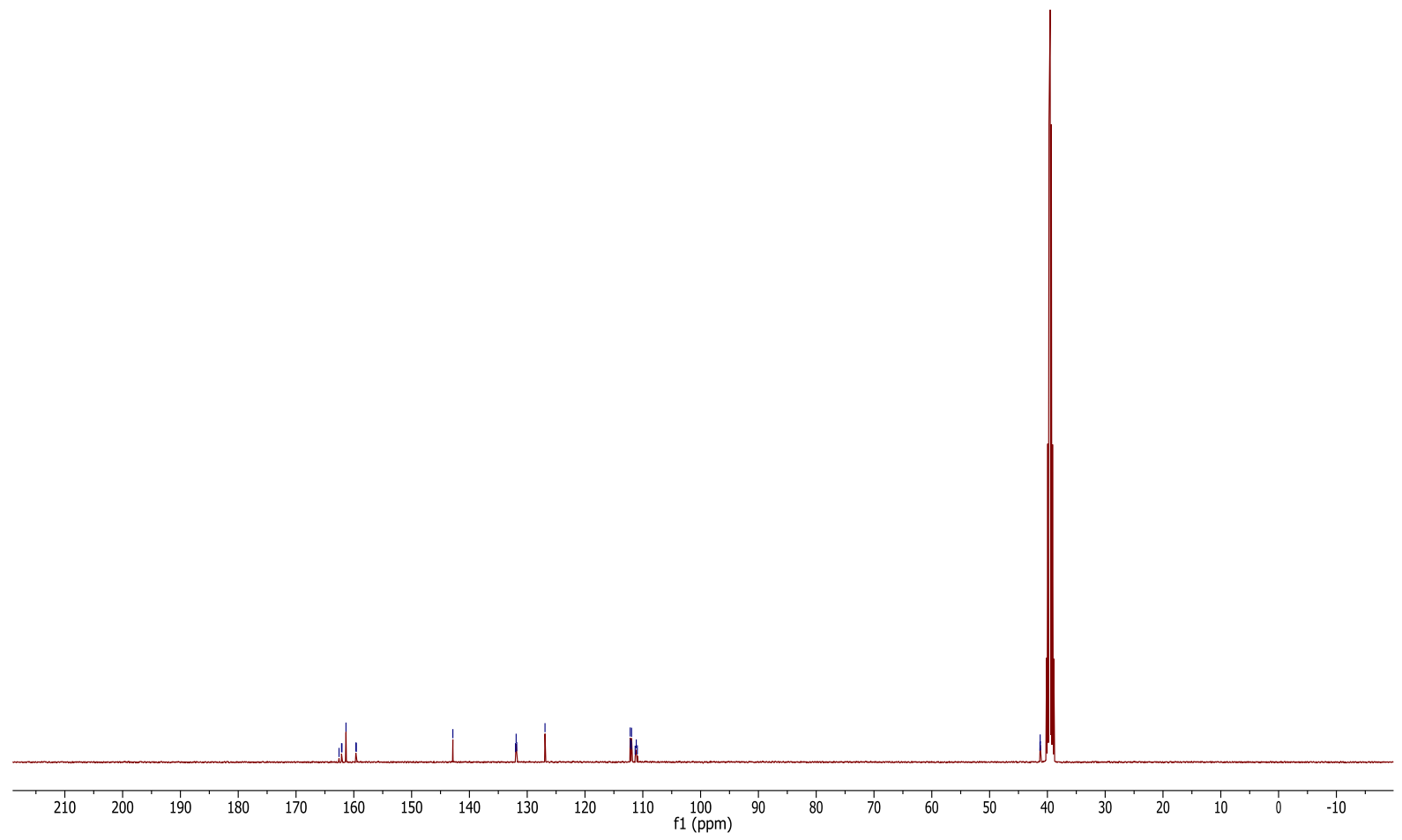

19F, $376 \mathrm{MHz}$, (CD3)2SO

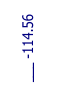

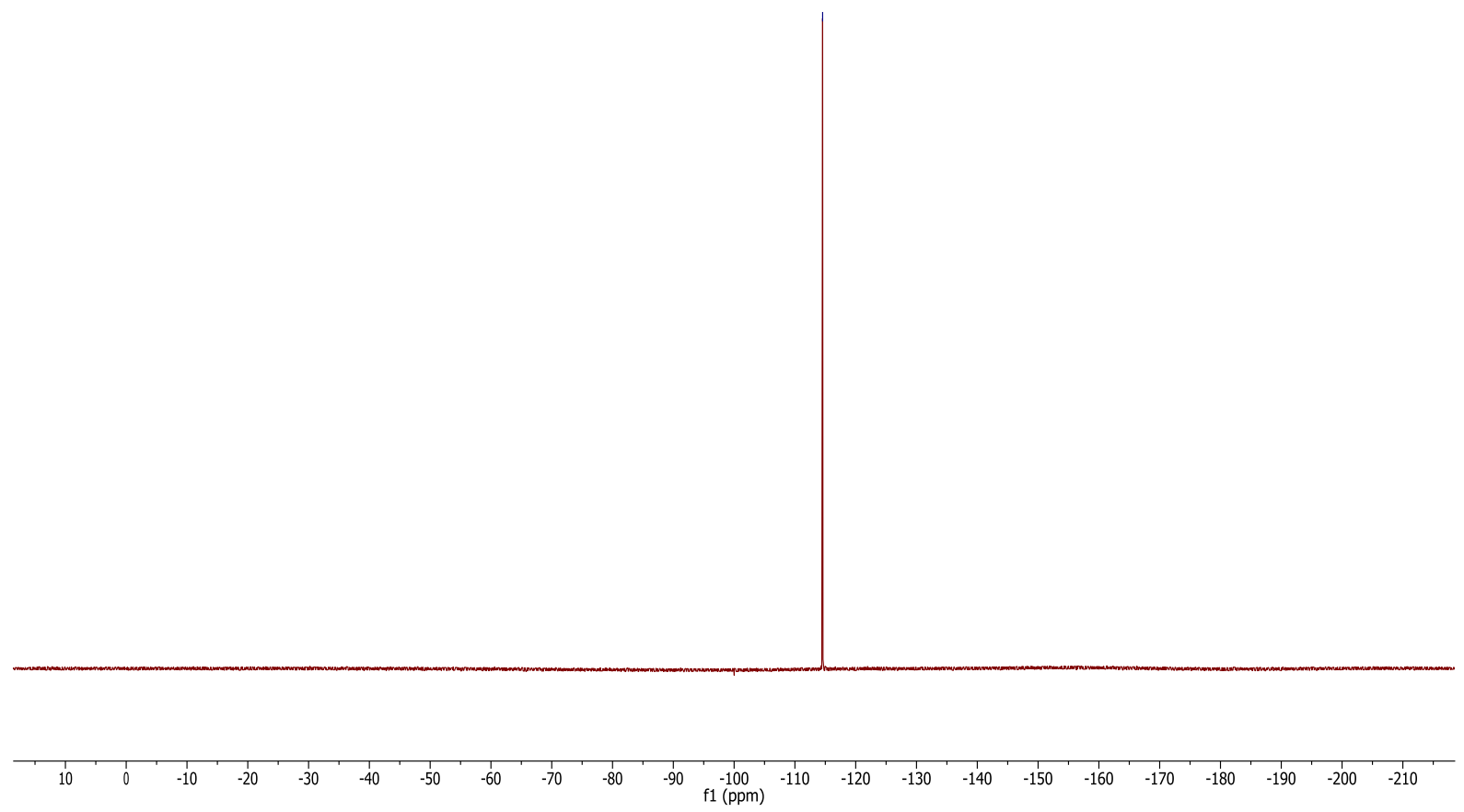

S49 


\section{References}

[1] Pirtsch, M.; Paria, S.; Matsuno, T.; Isobe, H.; Reiser, O. Chem. Eur. J. 2012, 18, 7336-7340.

[2] Vita, M. V.; Waser, J. Org. Lett. 2013, 15, 3246-3249.

[3] Ramella, V.; He, Z.; Daniliuc, C. G.; Studer, A. Org. Lett. 2015, 17, 664-667.

[4] Bräse, S.; Gil, C.; Knepper, K.; Zimmermann, V. Angew. Chem. Int. Ed. 2005, 44, 5188-5240.

[5] Zhdankin, V. V.; Kuehl, C. J.; Krasutsky, A. P.; Formaneck, M. S.; Bolz, J. T. Tetrahedron Lett., 1994, 35, 9677-9680.

[6] Rossy, C.; Majimel, J.; Delapierre, M. T.; Fouquet, E.; Felpin, F.-X. J. Org. Chem. 2014, 755, 78-85.

[7] Howson, S. E.; Clarkson, G. J.; Faulkner, A. D.; Kaner, R. A.; Whitmore, M. J.; Scott, P. J. Chem. Soc. Dalton Trans. 2013, 42, 14967-14981.

[8] Lamani, M.; Prabhu, K. R. Angew. Chem. Int. Ed. 2010, 49, 6622-6625.

[9] Pinto, O.; Lameiras, M.; Dias, L.; Rodrigues, M.; Santos, D.; Fernandez, C. J. Mol. Struct. 2010, 980, 163171.

[10] Yuan, Y.; Kwok, R. T. K.; Tang, B. Z.; Liu, B. J. Am. Chem. Soc. 2014, 136, 2546-2554.

[11] Chun, J.-H.; Pike, V. W. Eur. J. Org. Chem. 2012, 24, 4541-4547.

[12] Matake, R.; Niwa, Y.; Matsubara, H. Org. Lett. 2015, 17, 2354-2357.

[13] Maddani, M.; Prabhu, K. R. Tetrahedron Lett. 2008, 49, 4526-4530.

[14] Siles, R.; Kawasaki, Y.; Ross, P.; Freire, E. Bioorg. Med. Chem. Lett. 2011, 21, 5305-5309.

[15] Bristol-Myers Squibb Company, Patent US5116833 A1, 1992.

[16] Pramanik, S.; Ghorai, P. Org. Lett. 2014, 16, 2104-2107.

[17] Wright, A. T.; Zhong, Z.; Anslyn, E. V. Angew. Chem. Int. Ed. 2005, 44, 5679-5682.

[18] Kulkarni, S. S.; Hu, X.; Manetsch, R. Chem. Comm. 2013, 49, 1193-1195.

[19] Colombano, G.; Albani, C.; Ottonello, G.; Ribeiro, A.; Scarpelli, R.; Tarozzo, G.; Daglian, J.; Jung, K.-M.; Piomelli, D.; Bandiera, T. ChemMedChem 2015, 10, 380-395.

[20] Lee, J. H.; Gupta, S.; Jeong, W.; Rhee, Y. H.; Park, J. Angew. Chem. Int. Ed. 2012, 51, 10851-10855.

[21] Ikeda, H.; Hoshi, Y.; Namai, H.; Tanaka, F.; Goodman, J. L.; Mizuno, K. Chem. Eur. J., 2007, 13, 9207-9215.

[22] Schuster, E. M.; Botoshansky, M.; Gandelman, M. Angew. Chem. Int. Ed. 2008, 47, 4555-4558.

[23] Colombano, G.; Albani, C.; Ottonello, G.; Ribeiro, A.; Scarpelli, R.; Tarozzo, G.; Daglian, J.; Jung, K.M.; Piomelli, D.; Bandiera, T. ChemMedChem, 2015, 10, 380-395. 